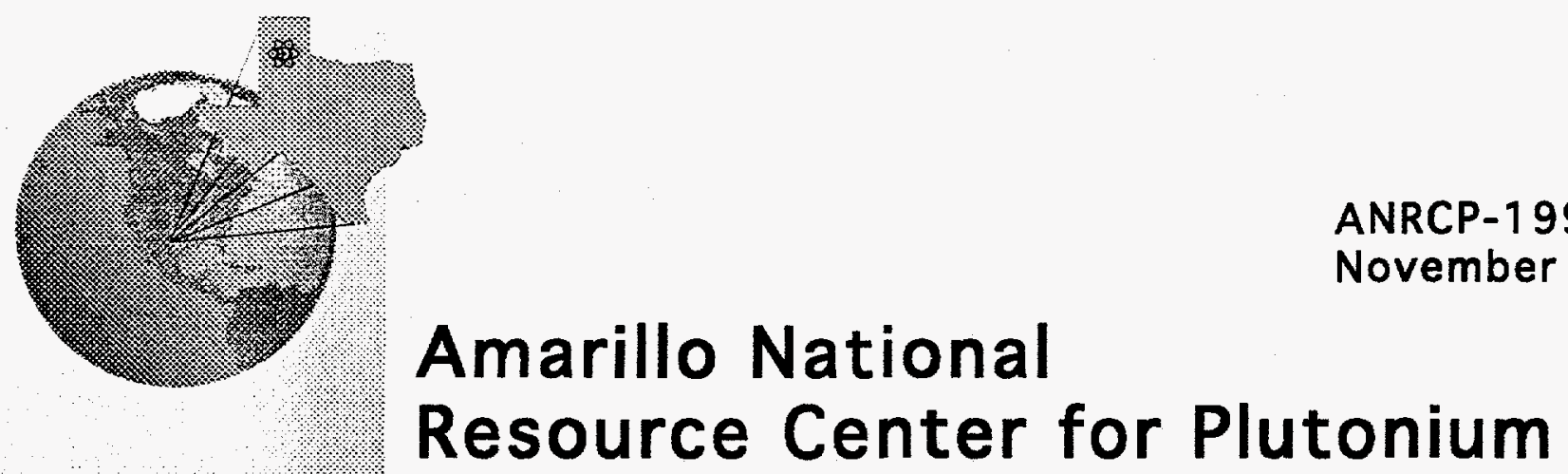

A Higher Education Consortium of The Texas A\&M University System, Texas Tech University, and The University of Texas System

\title{
A Computational Study of Highly Viscous Impinging Jets
}

\author{
RECEIVED \\ Mark W. Silva \\ Department of Mechanical Engineering \\ . The University of Texas \\ NOV 301998 \\ OSTI
}

This report was prepared with the support of the US Department of Energy (DOE) Cooperative Agreement No. DE-FCO4-95AL8583Z

However, any opinions, findings conclusions, or recommendations? expressed herein we those of the author(s) and do $\mathrm{not}$. necessarily reflect the views of $D O E$ This work was cont ducted through the Amarillo National Resource Center fot Plutonium.

\section{DISTRIBUTION OF THS DOCUMENT IS UNMMTED \\ Edited by \\ Angela L. Woods \\ Technical Editor}

600 South Tyler • Suite 800 - Amarillo, TX 79101

(806) 376-5533 • Fax: (806) 376-5561

http://www.pu.org 


\section{DISCLAMMER}

This report was prepared as an account of work sponsored by an agency of the United States Government. Neither the United States Government nor any agency thereof, nor any of their employees, makes any warranty, express or implied, or assumes any legal liability or responsibility for the accuracy, completeness, or usefulness of any information, apparatus, product, or process disclosed, or represents that its use would not infringe privately owned rights. Reference herein to any specific commercial product, process, or service by trade name, trademark, manufacturer, or otherwise does not necessarily constitute or imply its endorsement, recommendation, or favoring by the United States Government or any agency thereof. The views and opinions of authors expressed herein do not necessarily state or reflect those of the United States Government or any agency thereof. 


\section{DISCLAIMER}

Portions of this document may be illegible in electronic image products. Images are produced from the best available original document. 


\title{
AMARILLO NATIONAL RESOURCE CENTER FOR PLUTONIUM/ A HIGHER EDUCATION CONSORTIUM
}

\author{
A Report on \\ A Computational Study of Highly Viscous Impinging Jets
}

\author{
Mark William Silva, B.S. \\ Department of Mechanical Engineering \\ The University of Texas at Austin \\ Austin, Texas 78712
}

Submitted for publication to

Amarillo National Resource Center for Plutonium

November 1998 


\section{ACKNOWLEDGEMENTS}

I would like to express my appreciation to my research advisor, Dr. Kenneth Ball, for his experience and suggestions, and his guidance in conducting this research and preparing this thesis. I would also like to thank Dr. Eric Taleff, the second reader of this thesis, and the other members of "Team Glass" (Dr. Theodore Bergman, Dr. Myungho Song, Dan Gagnon, Mike Gomon, and Brian Powers), for their insight and advice during our weekly meetings.

This thesis was prepared with the support of the U.S. Department of Energy (DOE), Cooperative Agreement No. DEFC04-95AL85832. It is based upon work supported under a National Science Foundation (NSF) Graduate Fellowship. However, any opinions, findings, conclusions, or recommendations expressed herein are those of the author and do not necessarily reflect the views of DOE or NSF. This work was conducted through the Amarillo National Resource Center for Plutonium. I thank the ANRCP and the NSF for their financial support of my work.

I appreciate all the help I received from Carol Bronisz and Judith Horwitz, CFD consultants for FLOW-3D and FIDAP, respectively. Both provided helpful guidance and advice regarding the use of the software. The engineering instruction I have received from various professors at Trinity University and The University of Texas at Austin is very much appreciated.

During my years at the University, I have enjoyed the friendship of several of the Thermal/Fluids graduate students, including Ikram Ahmed, Marcela Arana, Melvyn Fernandez, Francis França, Dan Gagnon, Mike Gomon, Clark Havis, Roger Hill, Gary Holtzman, Carol Hsu, Mike Kandis, DahChyi Kuo, Chao-Ho Lan, Sang Ho Lee, Maşahiko Matsumura, and Juan Carlos Morales.

Finally, all the members of my family are very dear to me, and I appreciate all the love they have provided. I am especially grateful to my parents for all they have given to me over the years, and to Tiffany Wiescamp for her consistent support and encouragement during my work on this project. I also thank God for providing me with the opportunity and talent to accomplish this task.

Mark W. Silva

The University of Texas at Austin

December 5, 1997 


\title{
A Computational Study of Highly Viscous Impinging Jets
}

\author{
Mark William Silva, M.S.E. \\ The University of Texas at Austin
}

Supervisor: Kenneth S. Ball

\begin{abstract}
Two commercially-available computational fluid dynamics codes, FIDAP (Fluent, Inc., Lebanon, NH) and FLOW-3D (Flow Science, Inc., Los Alamos, NM), were used to simulate the landing region of jets of highly viscous fluids impinging on flat surfaces. The volume-of-fluid method was combined with finite difference and finite element approaches to predict the jet behavior. Several computational models with varying degrees of physical realism were developed, and the results were compared with experimental observations.

In experiments, the jet exhibited several complex behaviors. As soon as it exited the nozzle, the jet began to neck down and become narrower. When it impacted the solid surface, the jet developed an instability near the impact point and buckled to the side. This buckling became a spiraling motion, and the jet spiraled about the impact point. As the jet spiraled around, a cone-shaped pile was built up which eventually became unstable and slumped to the side. While all of these behaviors were occurring, air bubbles, or voids, were being entrapped in the fluid pool.

The results obtained from the FLOW3D models more closely matched the behavior of real jets than the results obtained from the FIDAP models. Most of the FLOW-3D models predicted all of the significant jet behaviors observed in experiments: necking, buckling, spiraling, slumping, and void entrapment. All of the FIDAP models
\end{abstract}

predicted that the jet would buckle relatively far from the point of impact, whereas the experimentally observed jet behavior indicates that the jets buckle much nearer the impact point. Furthermore, it was shown that FIDAP is incapable of incorporating heat transfer effects into the model, making it unsuitable for this work. 


\section{TABLE OF CONTENTS}

1. INTRODUCTION AND BACKGROUND

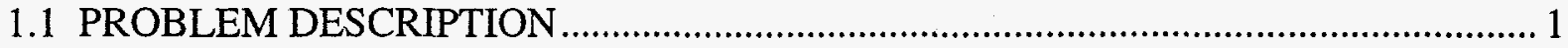

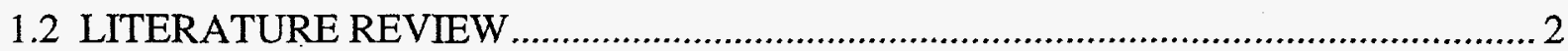

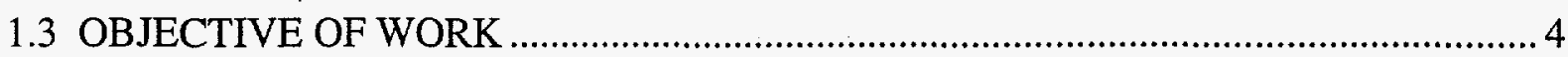

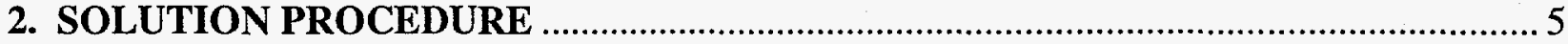

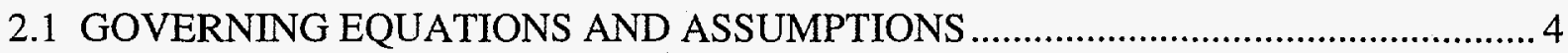

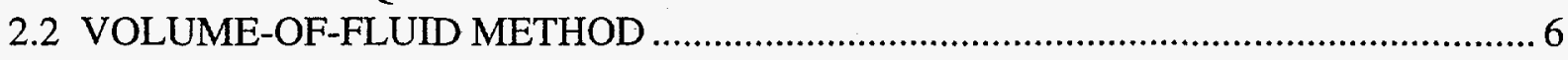

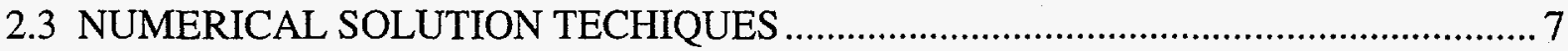

2.3.1 Finite Difference Method .................................................................................

2.3.2 Finite Element Method …………………………............................................... 9

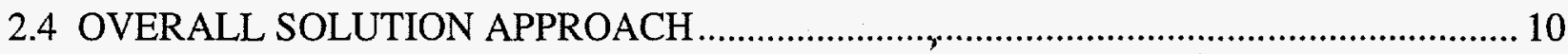

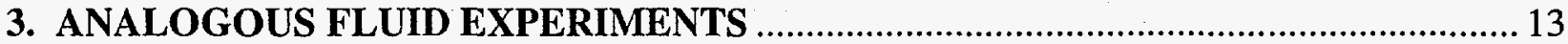

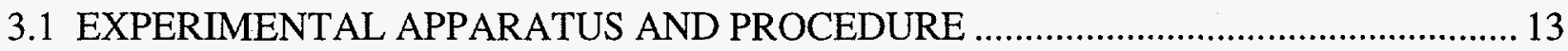

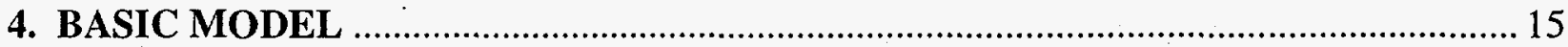

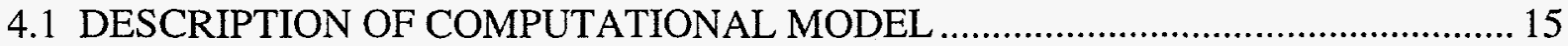

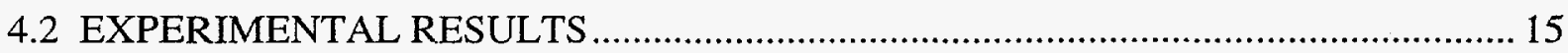

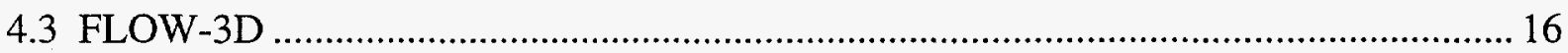

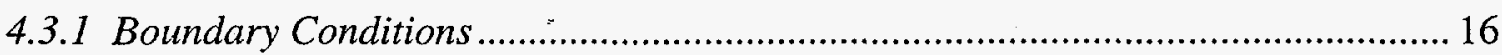

4.3.2 60,000 cSt Silicone Oil Results..................................................................... 17

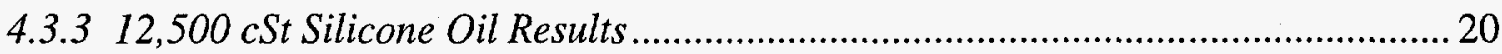

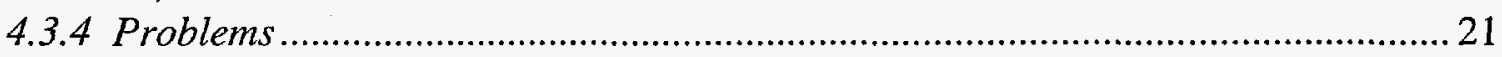

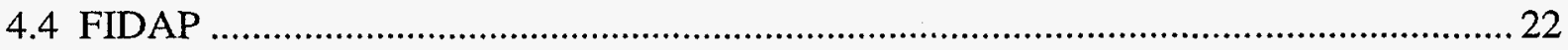

4.4.1 Boundary and Initial Conditions.................................................................. 22

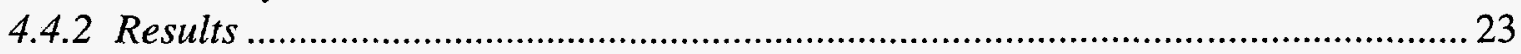

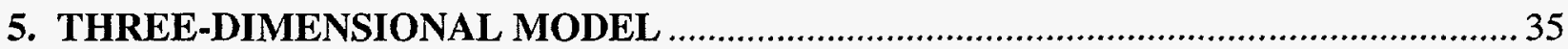

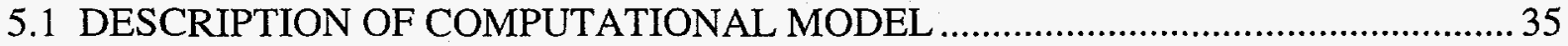

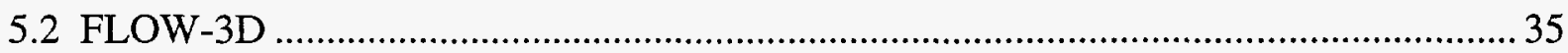

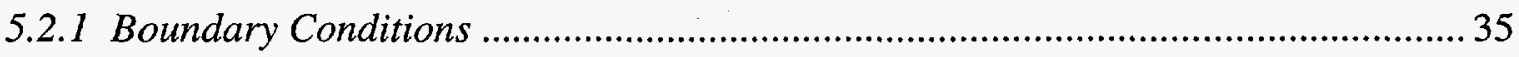

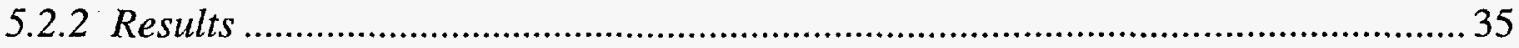

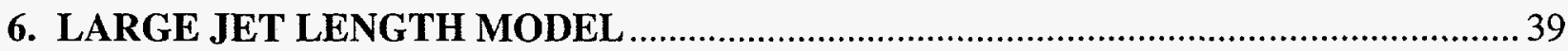

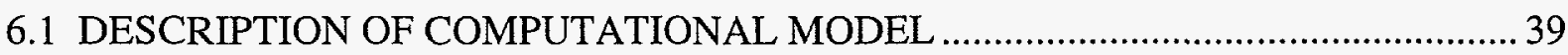

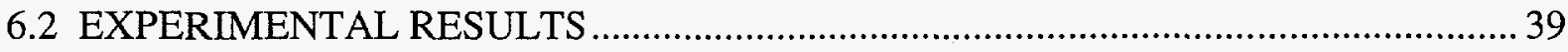

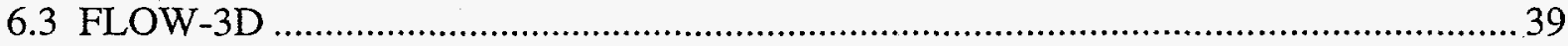

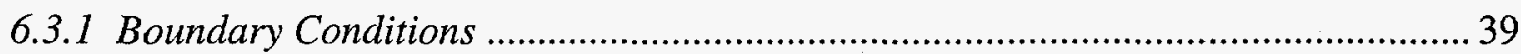

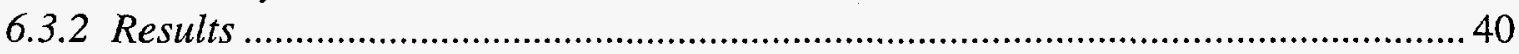

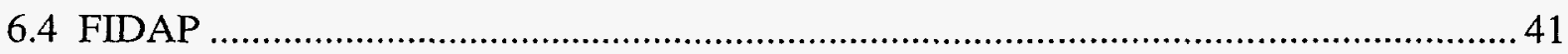

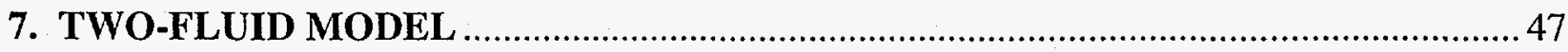


7.1 DESCRIPTION OF COMPUTATIONAL MODEL

7.2 FLOW-3D

7.2.1 Boundary Conditions .

7.2.2 Results

7.3 FIDAP

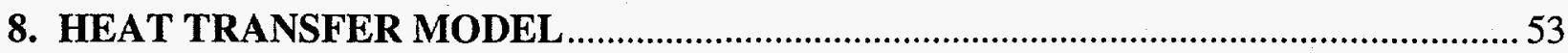

8.1 DESCRIPTION OF COMPUTATIONAL MODEL ……...........................................5

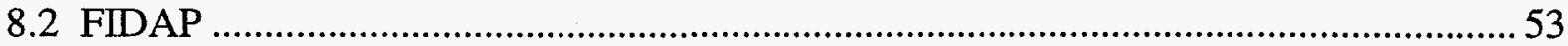

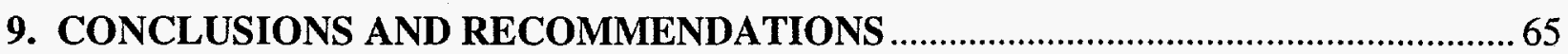

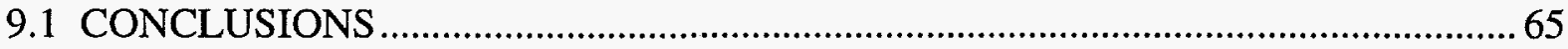

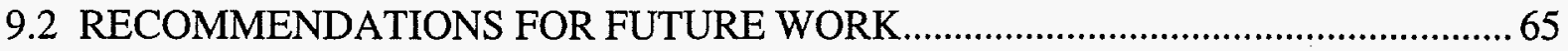

REFERENCES

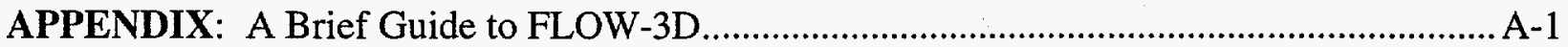




\section{LIST OF TABLES}

Table 4.1: Results of Small Jet Length Experiments .................................................... 24

Table 8.1: Effect of Thermal Conductivity on Temperature Error..........................................59 


\section{LIST OF FIGURES}

Figure 1.1: Various Flow Behaviors in Landing Region ........................................................ 4

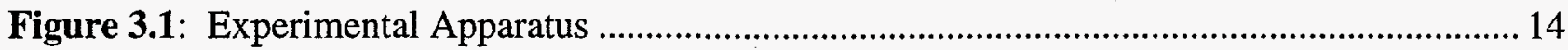

Figure 4.1: Close-Up of Velocity Vectors and Free Surface Position of 60,000 cSt Silicone Oil Flowing Through Vertical Pipe.

Figure 4.2: Typical FLOW-3D Results for 60,000 cSt Silicone Oil with Short Jet Length ............26

Figure 4.3: Sequence of Six Consecutive Frames Showing One Period of Jet Oscillation for a Typical 60,000 cSt Silicone Oil Jet with Short Length

Figure 4.4: Effect of Vertical Grid Resolution on Frequency of Jet Oscillation.............................2 28

Figure 4.5: Effect of Horizontal Grid Resolution on Frequency of Jet Oscillation .........................29

Figure 4.6: Effect of ITVSMX on Frequency of Jet Oscillation .......................................................30

Figure 4.7: Free Surface Behavior for Different Fluids ............................................................... 31

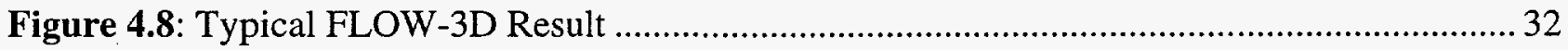

Figure 4.9: Typical FIDAP Results for 60,000 cSt Silicone Oil with Short Jet Length .................. 33

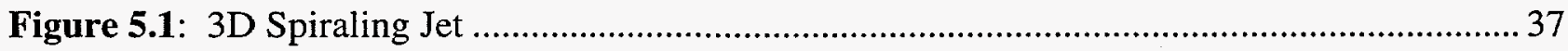

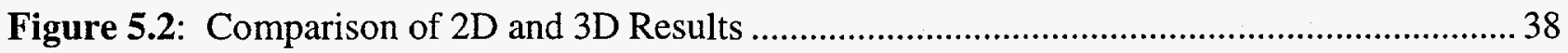

Figure 6.1: Typical FLOW-3D Results for 60,000 cSt Silicone Oil with Large Jet Length............ 43

Figure 6.2: Unrealistic FLOW-3D Results with Large Jet Length Model ....................................... 44

Figure 6.3: Comparison of FIDAP and FLOW-3D Results for Large jet Length Model ................45

Figure 7.1: Typical FLOW-3D Results for Two-Fluid Model....................................................50

Figure 7.2: Close-Up of Jet Tip during Free-Fall, Demonstrating the Effect of Surface Tension in FIDAP .

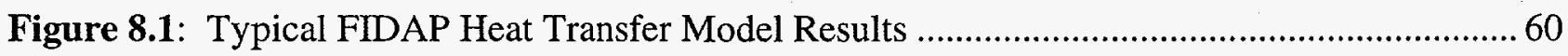

Figure 8.2: Typical FIDAP Heat Transfer Model Results ............................................................ 61 
Figure 8.3: Example of "Cellular" Temperature Distribution..............................................62

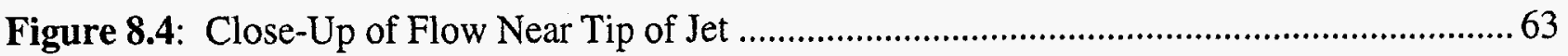




\section{NOMENCLATURE}

$c_{\mathrm{p}} \quad$ constant-pressure specific heat

Co Courant number

$e \quad$ internal energy

$F \quad$ fill fraction

$F \quad$ body force vector

F vector containing effects of body forces and boundary conditions

g gravitational acceleration vector

$i \quad$ element number

$k \quad$ thermal conductivity

K global system matrix

$M \quad$ Mach number

$P \quad$ pressure

$P e \quad$ cell Peclet number

$q \quad$ heat flux vector

$t$ time

$\Delta t \quad$ time step size

$T \quad$ temperature

$T_{0} \quad$ reference temperature

$u \quad$ horizontal component of velocity

$\boldsymbol{u} \quad$ velocity vector

$\mathbf{U}_{\mathrm{i}} \quad$ global vector of unknowns at current time step

$\mathbf{U}_{\mathrm{i}+1} \quad$ global vector of unknowns at future time step (with relaxation)

$\mathbf{U}^{*} \quad$ global vector of unknowns at future time step (without relaxation)

$v \quad$ vertical component of velocity

$x$ horizontal coordinate direction, or horizontal position 
$\Delta x \quad$ element width

y second horizontal coordinate direction

$z \quad$ vertical coordinate direction

$\alpha \quad$ acceleration factor

$\beta \quad$ coefficient of thermal expansion

$\varepsilon \quad$ convergence criterion

$\varepsilon_{\mathrm{f}} \quad$ cut-off to clean up "misty" elements

$\varepsilon_{\mathrm{r}} \quad$ convergence criterion for residual

$\varepsilon_{\mathrm{s}} \quad$ convergence criterion for solution

$\Phi$ viscous dissipation function

$\mu \quad$ dynamic viscosity

$\rho \quad$ density

$\tau \quad$ viscous stress tensor 


\section{INTRODUCTION AND BACKGROUND}

\subsection{PROBLEM DESCRIPTION}

The United States Department of Energy is considering several options for the immobilization of surplus weapons-grade plutonium. One option, known as the "can-incanister" option, involves mixing the plutonium with molten glass, pouring the mixture into small steel cans, and allowing it to solidify. Several of these cans are then suspended in a large canister, and a jet of molten glass and radioactive waste is poured into the canister to fill it. This can-in-canister filling procedure will be performed at the Defense Waste Processing Facility (DWPF).

This can-in-canister option is only one of several plutonium disposition options being considered by researchers at the Amarillo National Resource Center for Plutonium (ANRCP). Other disposition options include the "deep borehole" option, in which the plutonium is buried in a very deep hole, and the "energy production" option, in which the plutonium is used in a reactor to generate useful electricity.

The purpose of the ANRCP is to advance the science and technology, advise decision makers and inform the public on issues of concern to the Texas Panhandle region, the state of Texas, and the U.S. Department of Energy in the use and disposition of materials from nuclear weapon disassembly (ANRCP, 1996). This purpose is accomplished through a variety of tasks, such as developing a state-of-the-art Electronic Resource Library to archive information on nuclear weapons material; conducting environmental studies in areas including groundwater treatability, bioremediation, risk assessment, atmospheric pollution, and pathway analysis; communicating with the public through informational videos, public service announcements, technical brochures, and other means; educating the public through a student research conference, a graduate assistantship, research and technology laboratories, a K-16 education project, and a variety of other methods; and performing studies of nuclear weapon materials storage issues, such as robotics, air surveillance, pit encapsulation, storage containers, and aerosol dispersal.

The University of Texas (UT) at Austin is involved in this project in an effort to better understand the behavior of the molten DWPF glass as it fills the canister during can-in-canister pours. This goal is being pursued through four separate research projects at the UT. In one project, surrogate glass (glass which has properties that are similar to the DWPF glass, without the dangerous level of radioactivity) is melted in a small furnace and poured into a container, to simulate the actual can-in-canister pour on a laboratory scale. Another project involves developing a computer code to model the entire can-in-canister system, to predict the temperature distribution within the falling glass jet and the glass pool as the canister is filled. In a third project, experiments are performed at room temperature with analogous fluids (corn syrup and silicone oils, which have properties at room temperature that are similar to those of molten DWPF glass at high temperature) to safely and easily simulate the flow of molten glass into the canister. Finally, in the work that is the topic of this thesis, a detailed computational model of the "landing region" of the jet (the small region near the point of contact between the falling jet and the pool of fluid which has collected in the container) has been developed, to give insight into the fluid behavior and heat transfer in this region of the flow.

In both of the experimental projects described above (high-temperature pouring of surrogate glass, and room-temperature pouring of analogous fluids), the fluids exhibit several complex behaviors in the landing region. The behavior that is first observed is the buckling of the fluid jet. When it first impacts the bottom of the container, the jet develops an instability near 
the impact point and buckles to the side (like a solid column under a compressive load), instead of remaining axially symmetric. This buckling soon becomes a spiraling motion, and the jet spirals about the impact point, similar to the behavior of honey landing on a table as it is poured from a jar. As the fluid spirals around, a cone-shaped pile is built up. Eventually this pile grows too large and a second instability is observed as the pile slumps to the side. While all of these complex behaviors are occurring, air bubbles, or voids, are being entrapped in the fluid pool.

Photographs of these phenomena, taken during an experiment performed with corn syrup, are shown in Figure 1.1. The goal of the detailed computational modeling is to predict these complicated phenomena (buckling, spiraling, slumping, and void entrapment) in the landing region, for the room-temperature analogous fluid experiments and the actual DWPF canin-canister molten glass pours.

As motivation for the development of this detailed model, there are several areas in which the model is uniquely able to provide increased understanding of the flow. For example, a detailed computational model can provide insight into the mechanism of void formation, showing in detail how the voids become entrapped in the liquid pool, and can also permit a detailed study of the void migration, tracking the movement of the voids once they have become entrapped in the glass. The model can be used to calculate precise quantitative temperature, velocity, and pressure data, in both the jet and the surrounding air, throughout the entire region of interest, as opposed to the relatively small number of points at which these values may be measured using experimental sensors. A tremendous advantage of a detailed computational model is that it allows different configurations to be evaluated with a minimum of time and expense. For example; the effect of changing the can geometry within the canister, heating the canister walls prior to pouring the glass, varying the glass flow rate, or changing the properties of the glass, may be investigated with very little additional effort. Finally, a detailed model enables the researchers to evaluate the relative importance of the different modes of heat transfer, for example, by removing the radiative heat transfer effects and observing how the flow changes.

\subsection{LITERATURE REVIEW}

The earliest study of "buckling flows" which appears in the fluid mechanics literature is a qualitative experimental study reported by G. I. Taylor (1968). He studied electrically-driven and gravity-driven jets of viscous fluids, as well as other related flows, all of which experienced instabilities which caused them to buckle. In the case of the jets, Taylor related the onset of jet buckling to the Euler instability in a thin solid column: as the load on the column (end compression) is increased, a point is reached at which less force is needed to bend the column than to compress it. At this point, the column buckles laterally instead of continuing to compress axially. Taylor suggested that axial compression in thin fluid jets plays the same role as end compression in thin solid columns.

The next significant study of buckling jets was performed by Cruickshank and Munson (1981). In their experiments, a highviscosity fluid flowed through an orifice to generate either an axisymmetric or a plane jet, which fell through air and impinged on a flat horizontal plate. Cruickshank and Munson observed that if the distance between the orifice and the plate was less than some critical value, the jet would retain its axially symmetric shape, but if this critical distance (the "buckling height") was exceeded, the jet would buckle and spiral. Like Taylor, they hypothesized that compressive axial stress and the slenderness of the jet were combining to produce the fluid mechanics analogue to the buckling of a thin solid column. Cruickshank and Munson postulated that the frequency of the jet's oscillation depended upon the distance from the orifice to the plate 
(the "length" of the jet), the acceleration of gravity, the diameter of the orifice, the volume flow rate of the fluid, the surface tension of the fluid, the density of the fluid, and the viscosity of the fluid. From these seven variables, they identified four important dimensionless parameters, which govern the spiraling frequency of the jet, and showed that two of these parameters are significant in determining the buckling height. They performed experiments over a wide range of values of each dimensionless parameter, and discovered two critical parameters that determine whether or not the jet will buckle: the ratio of the jet length to the orifice diameter must be larger than a critical value, and the Reynolds number must be smaller than a critical value.

In 1987, Bejan published a thorough review of the current status of the research in buckling flows. He summarized the findings of several studies which had investigated buckling flows in a wide variety of situations, both in the laboratory and in natural settings. He noted that in all of these buckling flows, the buckling wavelength is directly proportional to the jet diameter or thickness. Bejan also identified a criterion for the onset of buckling: the length of the jet must be greater than the buckling wavelength. This is similar to, but more general than, Cruickshank and Munson's length-to-diameter criterion for the onset of buckling. However, for the gravity-driven jet configuration which is being studied in this work, Bejan's criterion is practically useless due to the difficulty in measuring the wavelength of the oscillations, since they are compressed by the weight of the upstream fluid. Bejan sorts the theoretical work on fluid buckling into two groups: the "viscida" theory, which was pioneered by Buckmaster (1973), neglects the effects of fluid inertia; and the inviscid theory neglects the effects of fluid viscosity. The relative success of each approach at matching experimental observations suggests that the early buckling deformation is dominated by inertia, and the later evolution of the flow is dominated by viscous effects. Bejan covers much more material in his review article, which is irrelevant to this work.

Six years after Bejan's review, Tchavdarov et al. (1993) published a theoretical analysis of jet buckling. Their analysis included all of the significant physical effects that influence a real jet, and thus was not limited to the extreme cases of the viscida or inviscid theories. The aim of Tchavdarov et al. was to provide a theoretical description of Cruickshank and Munson's experimental data. Using linear stability analysis, Tchavdarov et al. obtained analytical and numerical solutions, which agreed well with Cruickshank and Munson's data. They also confirmed Taylor's hypothesis that the jet buckling is due to axial compression, a result that disagreed with the viscida theory.

All of the previously cited works were concerned with the onset of jet buckling and the buckling frequency, and thus they ignored the effects of heat transfer on the jet. However, the effects of heat transfer are very significant in the DWPF can-in-canister glass pours, and they must be included in the detailed computational model of the landing region. Only one paper from the literature dealing with high-temperature liquid jets, Chacha et al. (1994), was obtained. The researchers used a finite difference technique to study the behavior of a high-temperature (molten) liquid jet. The problem was simplified significantly by holding all the fluid properties constant, neglecting convection heat transfer between the jet and the air, assuming the jet is opaque, gray, and diffuse, and applying the boundary layer approximation to the governing equations. These simplifications permit Chacha et al. to obtain numerical results which agree well with their experimental data, but unfortunately the simplifications restrict the numerical technique to a limited class of problems, so it cannot be applied to the present work. 


\subsection{OBJECTIVE OF WORK}

The objective of this work is to generate a numerical model of the landing region of the DWPF glass jet, using commercially-available computational fluid dynamics (CFD) software. Since the effects of heat transfer are very significant in the actual can-in-canister pours, these effects should be included in the detailed model. If a 2D model is capable of predicting all of the significant physical phenomena, which are observed experimentally, then this model is acceptable. However, if the $2 \mathrm{D}$ model is incapable of predicting the significant phenomena, then the model should be extended to $3 \mathrm{D}$.

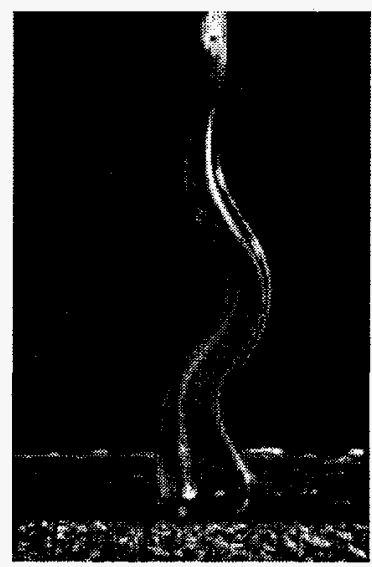

(a)

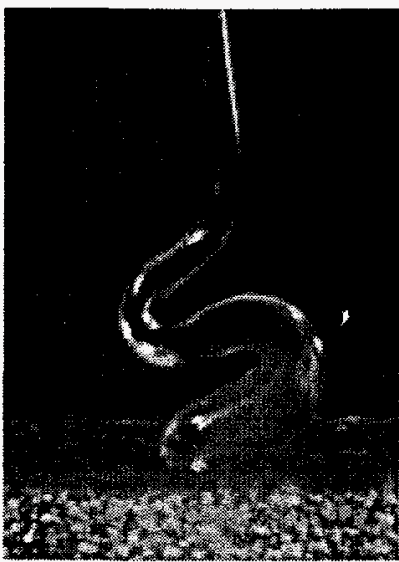

(b)

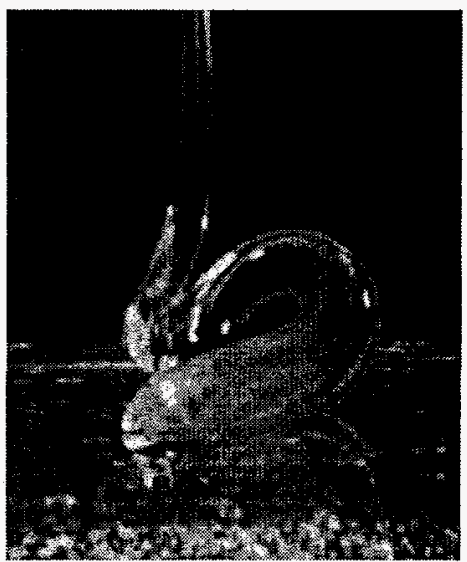

(c)

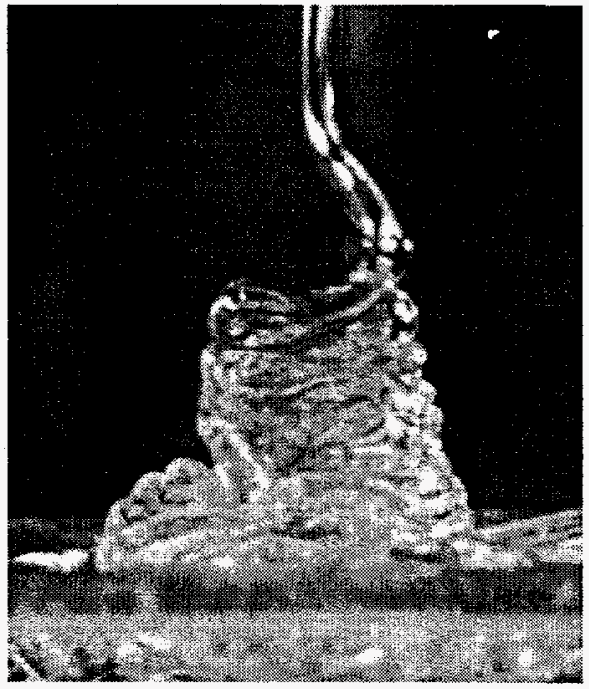

(d)

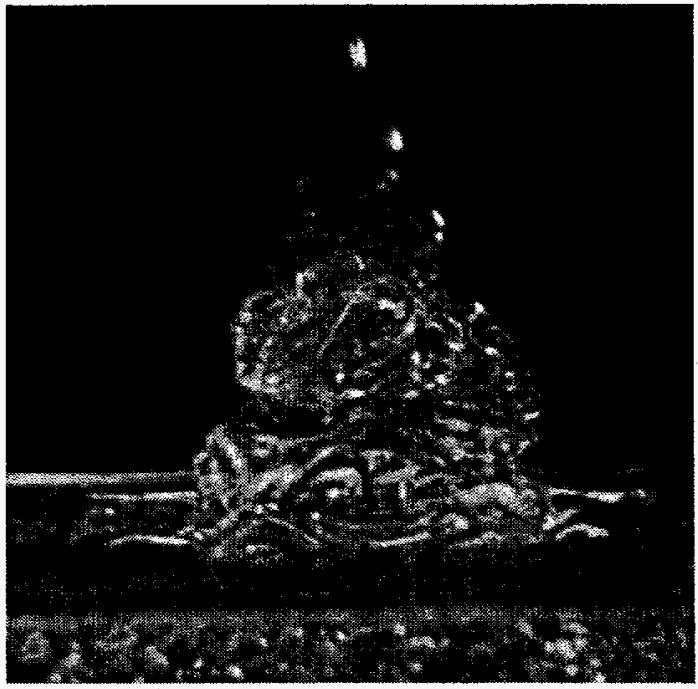

(e)

Figure 1.1: Various Flow Behaviors in Landing Region. Note voids entrapped in fluid pool in all pictures. (a) Jet buckling immediately after impact. (b) Further buckling. (c) Beginning of spiraling. (d) Further spiraling. (e) Cone slumping to left. 


\section{SOLUTION PROCEDURE}

\subsection{GOVERNING EQUATIONS AND ASSUMPTIONS}

The differential equations that govern the flow of a fluid are derived under the assumption that density and velocity are defined and continuous at every point in space. By conserving mass in an infinitesimal volume, the continuity or mass conservation equation is obtained:

$$
\frac{\partial}{\partial t}(\rho)+\nabla \cdot(\rho u)=0
$$

This equation indicates the balance between the rate of accumulation of mass in the volume and the flow rate of mass out of the volume.

Similarly, the conservation of momentum in an infinitesimal volume leads to:

$$
\frac{\partial}{\partial t}(\rho u)+\nabla \cdot(\rho u u)+\nabla P-\nabla \cdot \tau-\rho F=0
$$

In this equation, the rate of accumulation of momentum in the volume, the rate of convection of momentum into the volume, the pressure force acting on the volume, the viscous force acting on the volume, and the body force acting on the volume are all in balance.

Conserving the total energy in an infinitesimal volume yields the energy equation. After subtracting the mechanical energy from the total energy, the remaining terms form the thermal energy equation (Panton, 1996, p. 110):

$$
\frac{\partial}{\partial t}(\rho e)+\grave{\mathrm{E}} \cdot(\rho \mathbf{u} e)+P \grave{\mathrm{E}} \cdot \mathbf{u}-\mathrm{t}: \grave{\mathrm{E}} \mathbf{u}+\grave{\mathrm{E}} \cdot \mathbf{q}=0
$$

In words, the sum of the rate of accumulation of energy in the volume, the rate of convection of energy into the volume, the heating of the fluid by compression, the energy generated by viscous dissipation, and the net heat flux (including conduction and radiation), equals zero.
In their most general forms as shown in Equations $2.1-2.3$, the conservation equations apply to a wide variety of fluid flow and heat transfer situations. However, there are several approximations, which may be applied to these equations to limit them to the problem of interest. These approximations are: (1) the flow is incompressible; (2) the fluid is Newtonian; and (3) the Boussinesq approximation can be used to model the buoyancy force. In the following paragraphs each of these approximations will be explained, and then the final set of equations with all approximations applied will be given.

1. The main criterion for incompressible flow is that the Mach number must be low. In the room-temperature analogous fluid jet experiments, $M \sim 0.02$, which is well within the incompressible regime, and the Mach number has roughly this same value in the DWPF molten glass pours. Thus it is valid to approximate the jet flow as incompressible. This approximation greatly simplifies the governing equations in a number of ways. For example, the fluid density is constant in an incompressible flow.

2. Newtonian fluids have several special properties, which simplify the form of the viscous stress tensor $\tau$. For example, the stress is a linear function of strain rate, and the viscous stress tensor is symmetric (Panton, 1996, p. 132). Glass above its transition temperature is a Newtonian fluid (Viskanta, 1994), and corn syrup and silicone oil are also Newtonian (Suleiman and Munson, 1981). It may be noted that another important characteristic of Newtonian fluids is that their properties are isotropic (Panton, 1996, p. 132). This important simplification comes as a result of the Newtonian behavior of the fluid; it does not need to be assumed separately.

3. As explained above, the density is constant in a fluid, which is flowing incompressibly. However, without allowing for variable density, natural convection heat transfer is prohibited, since the variation in density with local temperature is the driving 
force for this type of heat transfer. The Boussinesq approximation includes a term in the energy equation which models the presence of a buoyancy force caused by density variation, while still retaining the constant density in the rest of the terms. In order to include natural convection effects in the fluid, the Boussinesq approximation will be applied.

Applying these three approximations to the governing equations yields:

$$
\begin{gathered}
\nabla \cdot \boldsymbol{u}=0 \\
\rho \frac{\partial \boldsymbol{u}}{\partial}+\rho \boldsymbol{u} \cdot \nabla \boldsymbol{u}+\nabla P-\mu \nabla^{2} \boldsymbol{u}+\rho g \beta\left(T-T_{0}\right)-\rho \boldsymbol{F}=0 \\
\rho c_{p} \frac{\partial T}{\partial t}+\rho c_{p} \boldsymbol{u} \cdot \nabla T-k \nabla^{2} T-\mu \Phi=0
\end{gathered}
$$

These three equations, the Navier-Stokes equations, are used to calculate the velocity $\boldsymbol{u}$, pressure $P$, and temperature $T$ within the fluid jet and, when necessary, within the surrounding air as well.

\subsection{VOLUME-OF-FLUID METHOD}

The Navier-Stokes equations are used to solve the kinematics and heat transfer within a fluid, but they provide no information about the location of the free surface which exists between the air and the fluid jet. To track this interface, the volumeof-fluid (VOF) method is used. In the VOF method, an additional degree of freedom $F$ is added to the problem. Each fluid in the problem is assigned a value of $F$. For this work, a value of $F=1$ corresponds to the jet fluid (corn syrup, silicone oil, or DWPF glass), and a value of $F=0$ corresponds to either the air surrounding the jet, or a constant-pressure void region which represents the environment. Every position in space has a value of $F$ which determines the fluid present at that location. Thus, the location of the free surface is the place where the value of $F$ jumps from 0 to 1 , indicating a change from air to jet fluid.

Tracking the motion of the free surface is a two-step procedure. The procedure starts with known values of $\boldsymbol{u}, P$, and $F$ at every point in space at time $t$. First, the Navier-Stokes equations are used to calculate $\boldsymbol{u}$ and $P$ at every point in space, at time $t+\Delta t$ in the immediate future. Since every point has a value of $F$, it is easy to determine which fluid is present at a given location, in order to decide which values to use for the fluid properties at that location. For locations with $0<F<1$, the fluid properties are interpolated linearly. Second, after the velocity at every point has been calculated, the value of $F$ at every point is carried along with the flow for time $\Delta t$, i.e., $F$ at a given point is moved through space according to the local velocity at that point to determine the new position of the free surface at time $t+\Delta t$. The movement of $F$ is calculated by an additional equation, which has the following form for an incompressible flow:

$\frac{\partial F}{\partial t}+\boldsymbol{u} \cdot \nabla F=0$

Now that the fluids have moved, the previous solution to the Navier-Stokes equations is no longer valid. Therefore the equations must be solved again, taking another small step $\Delta t$ into the future. This two-step procedure is repeated over and over to calculate the transient motion of the two fluids as the solution proceeds in time (FIDAP 7.5 Update Manual, 1995, p. 2-1).

The VOF method has one significant disadvantage: it is unable to represent exactly the precise position of the free surface. This shortcoming is due to the fact that the variable $F$, which is used to represent the relative distribution of the two fluids, does not permit a discontinuity, i.e. the value of $F$ cannot 
jump from 0 to 1 discontinuously at the free surface. Instead, a steep gradient in $F$ is used to represent the free surface, so the precise position of the free surface is somewhere in the "mushy zone" where $0 \leq F \leq 1$. Clearly this mushy zone is an unrealistic concept, since real, physical free surfaces are true discontinuities at the continuum level, so it is expected that the most accurate solution will be obtained when the thickness of the mushy zone is minimized. In this work, since the VOF method has been implemented on grids of discrete node points, the size of the mushy zone depends upon the distance between the grid points. Using more grid points allows them to be placed more closely together to determine the location of the free surface more precisely, at the expense of increasing the time required to obtain the solution. Many other free surface modeling techniques represent the free surface as the edge of the domain, so they do not suffer from this lack of precision.

However, the VOF method has a tremendous advantage over most other free surface modeling techniques: it permits very complex free surface deformations, including folding and break-up of the free surface. Since the goal of this work is to model the landing region, where the jet experiences very complex deformations as described in the previous chapter (buckling, spiraling, slumping, and void entrapment), the capability to simulate these free surface deformations is mandatory. Most other free surface modeling techniques cannot handle any free surface break-up or other complex deformations, so these methods are unacceptable for modeling the buckling jet problem. The VOF method's advantage of allowing complex deformations far outweighs its disadvantage of imprecise free surface position.

\subsection{NUMERICAL SOLUTION TECHNIQUES}

Since there is no known analytical solution to the set of governing Equations 2.4
- 2.7, approximate methods must be used to find a solution. Two numerical methods were used in this work: the finite difference method and the finite element method. The two methods are based on a similar principle. They both divide the continuum solution domain into a set of discrete points and approximate the governing differential equations to obtain a set of algebraic equations, which are solved iteratively to calculate the variables of interest at the discrete points. Once the solution has been obtained at a given time, the problem is advanced in time by a small amount and the solution is calculated again, to model the flow as it proceeds in time. The specific details of these two methods are explained in the following two subsections.

\subsubsection{Finite Difference Method}

FLOW-3D (Flow Science, Inc., Los Alamos, NM) is one of two commerciallyavailable CFD codes which were used in this work. It is based on the finite difference method. In this method, the solution domain is divided into a mesh of fixed rectangular cells. Each cell has a single value of $P, T$, and $F$, located at the center of the cell, and the velocities $\boldsymbol{u}$ are located at cell faces. To generate discrete approximate forms of the governing equations, each dependent variable is surrounded by a control volume, and the surface fluxes, surface stresses, and body forces acting on this control volume are expressed in terms of the dependent variables in the neighboring control volumes. Those fluxes, stresses, and body forces are used to approximate the various terms in the governing equations (FLOW-3D Theory Manual, p. 31).

The four-step algorithm to advance the solution through one time step $\Delta t$ is as follows (FLOW-3D Theory Manual, p. 34):

1. Compute a first guess to the new velocity field (at time $t+\Delta t$ ) based on the old solution (at time $\Delta t$ ) using the discrete form of 
the momentum conservation equation (Equation 2.5).

2. Adjust the pressure in each cell to satisfy mass conservation, Equation 2.4.

3. Compute the velocity changes induced by each pressure change, and add them to the velocities computed in step 1 .

4. Update the temperature and the position of the free surface, using the discrete forms of Equations. 2.6 and 2.7.

In this algorithm, Steps 2 and 3 are performed iteratively, since adjusting the pressure in one cell will upset the balance in the neighbor cells. These iterations may be performed using either the successive overrelaxation (SOR) technique, in which the solver sweeps through the domain updating the pressure in the cells one by one, or the alternating direction implicit (ADI) technique, in which the pressure is updated along an entire line of grid points simultaneously. Instead of satisfying Equation 2.4 exactly (requiring that its left-hand side equal exactly zero in every cell), the pressure iterations are continued until this equation is satisfied . approximately (the left-hand side is less than or equal to the convergence criterion $\varepsilon$ in every cell). The user chooses the value of $\varepsilon$, and the accuracy of the solution may be increased by choosing a smaller value for $\varepsilon$. Once the convergence criterion is satisfied in every cell, the pressure is considered to be the "correct" solution, and the algorithm proceeds to step 4.

The size of the time step $\Delta t$ is not chosen arbitrarily; on the contrary, there are several requirements which govern the maximum allowable size. These requirements are based on several non-dimensional numbers, which govern the stability of the solution. For example, the non-dimensional number, which determines the stability of the convective term in the momentum equation is the Courant number $\mathrm{Co}$ :

$$
C o=\frac{\Delta t u}{\Delta x}
$$

The value of $C o$ indicates the number of cells through which any convected quantity will travel in one time step. For the convective term to remain stable, it is required that $C o \leq$ 0.45 . If $C o$ is allowed to exceed this value, then the convective term will become unstable, i.e., the error will grow with successive iterations. Establishing this limit on the value of $\mathrm{Co}$ sets the maximum allowable value of $\Delta t$. Along with a value of $\mathrm{Co}$ in each coordinate direction, there are also non-dimensional numbers which govern the stability of the surface motion, surface tension forces, and viscous stresses, and each of these non-dimensional numbers places a restriction on the maximum value of $\Delta t$. To determine the value of $\Delta t$ to be used for a given time step, each relevant non-dimensional number is used to calculate a limit on the maximum permissible value of $\Delta t$ in each cell, and then the most restrictive one (the one which requires the smallest value of $\Delta t$ ) sets the value of $\Delta t$ for the time step (FLOW-3D Theory Manual, p. 73).

FLOW-3D offers two implementations of the VOF method, which will be labeled the "single-fluid" and "twofluid" approaches. In the two-fluid approach, cells with $F=1$ represent the jet fluid (corn syrup, silicone oil, or DWPF glass), and cells with $F=0$ represent the air surrounding the jet. In this approach, the governing equations are solved in every cell at each time step, to track the motion of both fluids (the air and the jet fluid). In the single-fluid approach, cells with $F=1$ again represent the jet fluid, but now cells with $F=0$ represent a constantpressure void region. This constant-pressure region is used only to provide a boundary condition at the free surface of the jet; there is no real fluid in this region since it has no properties defined, and the governing equations are solved only within the jet fluid, not in the constant-pressure region.

The single-fluid model has two main advantages over the two-fluid model. First, the single-fluid model requires much less time 
to obtain a solution than the two-fluid model, since the governing equations are solved only in cells containing the jet fluid, which for this work is a very small fraction of the computational domain. Second, the inclusion of the second fluid causes some numerical diffusion of the free surface, which decreases the accuracy of the solution. However, the two-fluid model has some advantages of its own. First, the heat transfer between the jet fluid and the air may be calculated by applying the energy conservation equation (Equation 2.6) throughout the entire domain. Second, the voids which are entrapped in the fluid pool will be true air bubbles, as opposed to the voids entrapped using the single-fluid model, which disappear when compressed since they have no density.

\subsubsection{Finite Element Method}

The other commercially-available CFD code used in this work is FIDAP (Fluent, Inc., Lebanon, NH), which is based on the finite element method. The solution domain is divided into a mesh of fixed rectangular elements. Within each element, the dependent variables $\boldsymbol{u}, P, T$, and $F$ are interpolated at a number of node points. This interpolation function is known as the basis function, and the order of the basis function : determines the number of node points present in each element. Approximating the continuous dependent variables as discrete, interpolated values introduces error into the governing equations, so the left-hand sides of the governing Equations $2.4-2.7$ will no longer equal zero. Rather, each equation will give a non-zero residual when the terms on the left-hand side are evaluated, and the exact, correct solution is approached as the value of each residual is decreased. To minimize the residuals, FIDAP uses the Galerkin form of the method of weighted residuals, which makes the residual for each degree of freedom orthogonal to its basis function. This orthogonality condition leads to a discrete vector form for each governing equation within each element. When the elements are assembled, and interelement continuity of the degrees of freedom is enforced, a single matrix equation is generated, and this equation is solved to obtain the value of each degree of freedom at each node (FIDAP 7.0 Theory Manual, p. 3-1).

Like F£OW-3D, FIDAP uses an iterative technique to obtain the solution at each time step. FIDAP offers several iterative solution techniques, including a successive substitution algorithm, a segregated algorithm, and a variety of Newton-Raphsonbased algorithms. The simplest technique, which was used in this work, is the successive substitution technique, also known as fixed point iteration or Picard iteration. To calculate the unknown values of the dependent variables for a certain iteration, all of the coefficients in the matrix equation are evaluated using the known values of the dependent variables from the previous iteration (Reddy and Gartling, 1994, p. 95). When the coefficients are evaluated in this manner, the only unknown parameter in the problem is a column vector containing the values of the dependent variables, so the matrix equation may easily be solved to determine these values. Once these values have been obtained for a given iteration, they are compared to the values of the dependent variables from the previous iteration, using a root-mean-square norm. If the values differ by less than a user-specified value $\varepsilon$, then the solution has converged for that time step, and the problem proceeds to the next time step; otherwise, another iteration is performed.

Recall that, as described in the previous section, FLOW-3D determines the size of the time step by evaluating several stability criteria and choosing the most restrictive one. These stability criteria are required because FLOW-3D uses an explicit method, which is only stable under certain conditions, to advance the solution in time. FIDAP provides several methods to model the transient portion of the solution, and in this work, an implicit method (first-order backward Euler), which is unconditionally 
stable, was used to advance the solution of Equations $2.4-2.6$ in time. Since the method is unconditionally stable, the time step may be made as large as desired. Increasing the size of the time step will decrease the accuracy of the solution, but it will not cause the solution to diverge, as is the case when using an explicit method. However, after solving Equations $2.4-2.6$ to update $u, P$, and $T$, FIDAP uses an explicit method to update the free surface position using Equation 2.7, and the use of this explicit method does place a stability criterion on the time step size, namely the Courant criterion of Equation 2.8.

Like FLOW-3D, FIDAP offers both the single-fluid and two-fluid models to implement the VOF method. These models have the same advantages and disadvantages in their finite element FIDAP implementation as they do in their finite difference FLOW-3D implementation.

\subsection{OVERALL SOLUTION APPROACH}

In developing the detailed computational model, there are several . physical effects that may or may not be included. In general, including an effect will cause the model to more closely represent the actual physical process, but each effect will also make the problem more difficult or timeconsuming to solve computationally. Thus, the model will be developed in several consecutive stages. The first stage will be the least physically realistic but the easiest to solve. After this first-stage model has been compared with experimental data and found to produce correct results, the other physical effects will be added in stages, one by one, gradually building up to the final model, which will be a very good representation of the actual can-in-canister process.

The first stage of the model will satisfy the mass and momentum conservation equations and the VOF equation, but the energy conservation equation will not be included in the analysis. The model will be limited to two dimensions. A relatively small jet length will be used (i.e., the container into which the jet is flowing will be placed relatively near the fluid nozzle). The fluid properties will be chosen to correspond to one of the analogous fluids, rather than the molten glass. A single-fluid model will be used rather than a two-fluid model. This model will then be developed by adding the effects described below.

A simple way to develop the model will be to extend it to 3D. This will allow the model to resemble physical reality much more closely. However, the addition of the third physical dimension will greatly increase the time required to obtain a solution. It will be important to compare the $2 \mathrm{D}$ and $3 \mathrm{D}$ results to see if the $2 \mathrm{D}$ model adequately predicts all the important physical effects in the problem. If so, the $2 \mathrm{D}$ model can be used instead of the 3D model, which will lead to tremendous savings in the time required to solve the problem.

The small jet length will make the problem simpler to solve for several reasons. The experimental results of Cruickshank and Munson (1981) indicate that, in general, the spiraling frequency of the jet increases as the jet length is increased. A faster spiraling frequency means that the size of the time steps required for stability will be decreased, which will increase the amount of time required to obtain a solution. Also, physical intuition suggests that the jet will neck down and become narrower as it falls. As the jet becomes narrower, the problem becomes more difficult and time-consuming to solve, since the grid points must be clustered closer together to resolve the flow in the narrow jet. However, the jet lengths in the various physical processes being modeled are fairly long. The lab-scale molten glass experiments use a jet length of about $1 \mathrm{~m}$, the analogous fluid experiments have a jet length of slightly less than $2 \mathrm{~m}$, and the actual can-in-canister pouring process involves a jet length near 4 $\mathrm{m}$. Therefore, increasing the jet length, while making the problem more difficult to solve will also increase the realism of the model. 
The addition of the air to the model (switching from a single-fluid model to a twofluid model) has several very significant advantages, as discussed in Section 2.3.1. With a single-fluid model, the voids, which are entrapped by the oscillating jet, have no mass or density, so they disappear when compressed. The addition of air to the model will allow the void formation mechanisms to be studied in detail, to determine exactly how the voids become entrapped in the fluid pool. It will also permit the investigation of the voids' motion once they are entrapped. Another great advantage of the two-fluid model is that the convective heat transfer between the air and the jet can be included. With a single-fluid model, there is no way to simulate the heat transfer between the falling jet and the air, so the only heat transfer which can occur is conduction to the container which is filled by the fluid jet, and radiation to the receiving container and the surroundings. However, the two-fluid model does have two main drawbacks. The addition of the second fluid tends to increase numerical diffusion of the free surface position, introducing error into the solution. Also, the problem takes longer to solve, since the governing equations must be solved in every cell, rather than just the cells which contain the jet fluid.

When the energy conservation equation is added to the problem, the solution will provide information about the temperature in the fluids and the effects of heat transfer on the flow. These effects are expected to contribute very significantly to the solution. The main way that heat transfer will affect the flow is through the temperature-dependent viscosity of the DWPF glass and the corn syrup. The viscosities of both of these fluids are very strongly dependent on temperature: their viscosities increase by a factor of 1000 over the range of temperatures encountered in a typical experiment (Soper and Bickford, 1982; Chu and Hickox, 1990). (Their other properties are also functions of temperature, but the effect is not as strong.) This changing viscosity can affect the flow in a number of ways. For example, the motion of the voids in the fluid pool is expected to depend strongly on the temperature of the surrounding fluid. In addition to the temperature-driven natural convection affecting the void motion, the increasing viscosity of the cooling fluid pool will "lock" some of the voids into place. (A major goal of the can-in-canister research at UT is to minimize the amount of entrapped air, since it compromises the structural integrity of the solidified glass within the canister.) Also, the radiative and convective heat transfer from the outer surface of the jet may cause the viscosity of the jet's outer surface to be significantly lower than the viscosity at the center. This would create a sort of hard shell on the outside of the jet, which is expected to have a significant effect on the buckling instability and the spiraling frequency. Of course, there is a price to be paid in exchange for this temperature and heat transfer information: the addition of the thermal energy equation increases the amount of time required to obtain a solution. Also, since this equation is strongly coupled to the momentum conservation equation through the Boussinesq buoyant force, the viscous dissipation, and the temperature-dependent fluid properties, it increases the nonlinearity of the system of equations, thus making it more difficult to obtain a stable, converged solution.

The final, fully developed model will satisfy the mass, momentum, and energy conservation equations, along with the VOF equation, in three dimensions, for a large jet length chosen to correspond to the can-incanister process. It will include the effects of buoyancy and temperature-dependent fluid properties, and will solve the governing equations in both the air and the jet fluid. This model should produce a solution, which is quite realistic from a physical perspective, since it includes all the important physical effects. 



\section{ANALOGOUS FLUID EXPERIMENTS}

\subsection{PERIMENTAL APPARATUS AND PROCEDURE}

As mentioned in Chapter 1, a fellow University of Texas researcher performed a series of experiments with analogous fluids fluids whose properties (particularly viscosity) at room temperature are similar to the properties of the molten DWPF glass at high temperature. Three different analogous fluids were used in these experiments: $42 / 43$ corn syrup, Dow Corning 12,500 cSt silicone oil, and Dow Corning 60,000 cSt silicone oil. Thermophysical property data for the corn syrup was obtained from Chu and Hickox (1990), and data for silicone oil was obtained from the Dow Corning Corporation (1980).

Figure 3.1 shows a photograph and schematic of the apparatus used to perform these experiments. For each experiment, the hydraulic cylinder was filled with an analogous fluid. The motor was set to a constant power to drive the piston forward, which forced the fluid out of the cylinder and through the vertically-oriented round nozzle. The fluid then formed a jet, which fell vertically to land in a receiving container, a rectangular box of transparent acrylic, which was sitting on a scale.

In addition to making qualitative observations, several quantities were also measured to help describe the flow. The weight of fluid in the container was measured as a function of time, to determine the mass flow rate of the fluid. The velocity of the jet was measured by marking the fluid with food coloring dye, and capturing the motion of the marker points with a high-speed camera. The high-speed camera was also used to measure the spiraling frequency of the landing jet. At the end of an experiment, after the receiving container had been filled with analogous fluid, the weight of water displaced by the full container was measured, to determine the void fraction of the fluid in the container.

For more information on these analogous fluid experiments, see Gomon (1997). 


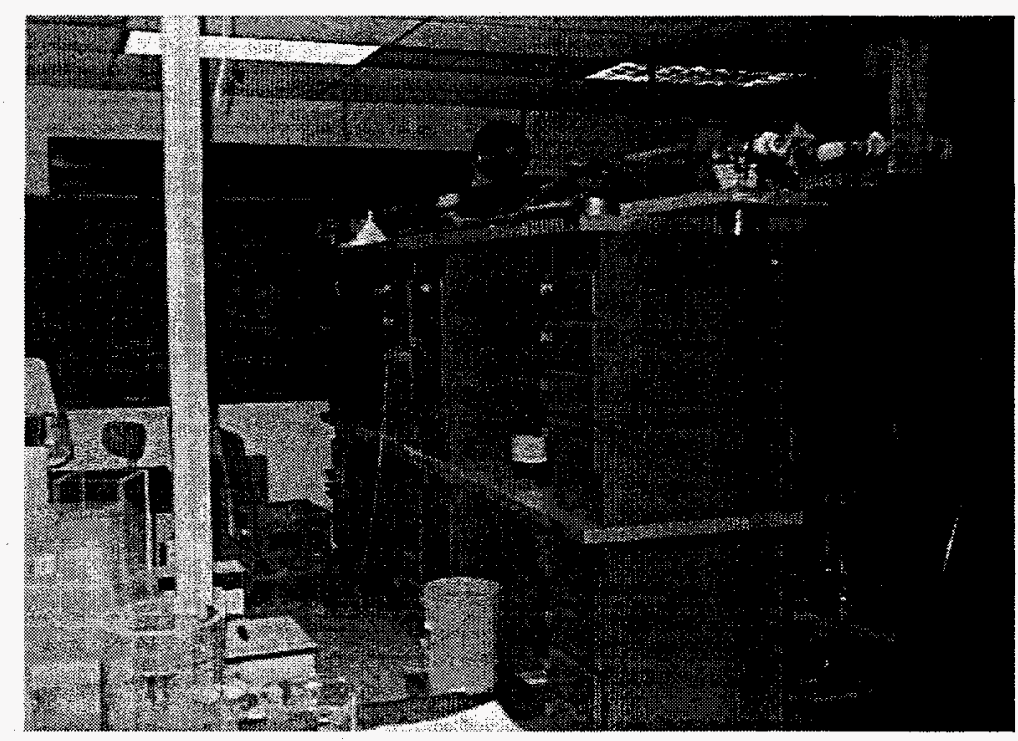

(a) Photograph

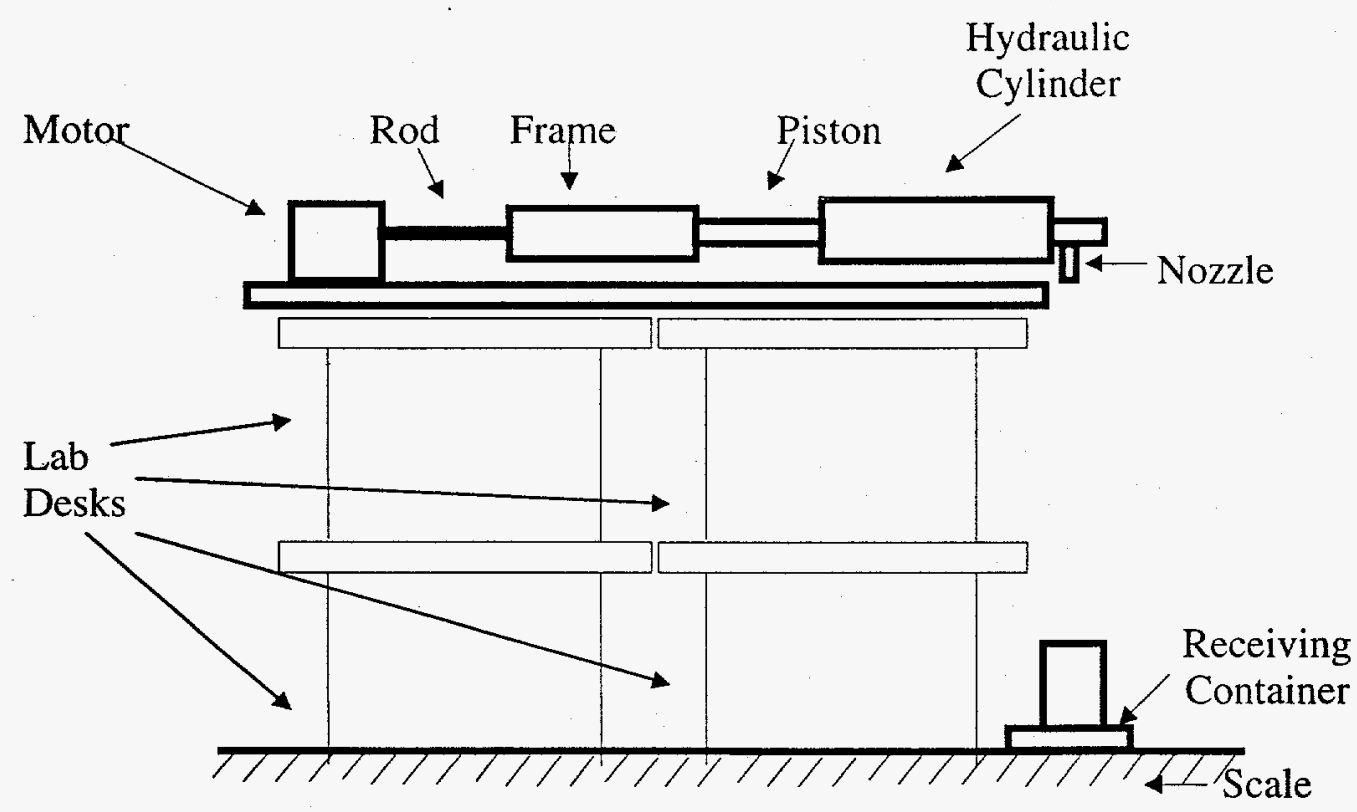

(b) Schematic

Figure 3.1: Experimental Apparatus. (a) Photograph. (b) Schematic. 


\section{BASIC MODEL}

\subsection{DESCRIPTION OF COMPUTATIONAL MODEL}

As explained in Section 2.4, the development of the computational model begins with the simplest model, which includes a minimal number of physical effects. The relevant effects will be added in stages to develop gradually the final model, which will be the most physically realistic. This chapter focuses on the "basic model," the simplest computational model, and in each successive chapter, one physical effect will be added to this model. The "basic model" which is the focus of this chapter has the following characteristics:

- two-dimensional,

- small jet length,

- single fluid, and

- isothermal.

\subsection{EXPERIMENTAL RESULTS}

The analogous fluid experiments were performed with the apparatus in two different configurations. One of these, which will be referred to as the "small jet length" configuration, had the receiving container placed $34 \mathrm{~cm}$ below the nozzle. Several qualitative observations of the flow behavior in this configuration were made. As the analogous fluid flowed through the nozzle and formed a jet, the jet diameter immediately began to neck down or narrow. This necking continued to decrease the jet diameter as the jet accelerated toward the receiving container. Once the jet impacted the container, the various physical phenomena described in Chapter 1 were all observed. The jet thickened slightly and buckled to the side, then began to spiral around the impact point.

A conical pile was built up which occasionally became unstable and slumped to the side. As the jet gradually filled the container, a multitude of small air voids became entrapped in the fluid pool.
For purposes of comparison with the computational model, it was decided to focus on the spiraling motion of the jet and measure the spiraling frequency. In this "small jet length" configuration, the spiraling frequency for a particular fluid was observed to depend upon both the mass flow rate of the fluid and the amount of fluid in the receiving container (the "pool height"). In fact, the frequency is actually a function of the jet diameter at the point where the buckling occurs, and for the short jet length configuration, both the mass flow rate and the pool height affect the jet diameter at the buckling location. The mass flow rate affects the jet diameter since an increase in the amount of mass flowing through the nozzle will increase the diameter of the jet. The pool height affects the diameter because the jet is necking down and becoming narrower as it falls, but it has not yet reached a steady diameter, so as the receiving container fills with fluid and the pool height increases, the diameter of the jet at the buckling location also increases as the jet length is effectively decreased.

Six experiments were performed with the apparatus in the small jet length configuration. Two experiments used 60,000 $\mathrm{cSt}$ silicone oil as the working fluid, and the other experiments were performed with $12,500 \mathrm{cSt}$ silicone oil. The results of these experiments are shown in Table 4.1. For most of the experiments, the length of time during which the pool height and frequency were measured was relatively short, and the pool height and frequency were observed to have constant values as shown in the table. For experiment \#2, however, the pool height and frequency were measured over a span of eight seconds, and the pool height and frequency changed significantly between the beginning and the end of this interval, so for this experiment, the results are presented as experiment \#2a (at the beginning of the eightsecond time interval) and experiment \#2b (at the end of the time interval). These experimental results will be used as the basis for a quantitative evaluation of the 
performance of the small jet length models described in the following sections.

\subsection{FLOW-3D}

\subsubsection{Boundary Conditions}

Since the receiving container in the analogous fluid experiments is rectangular, it was decided to model these experiments using a rectangular Cartesian grid system. The domain in the $x$ direction represented the width of the receiving container, and the domain in the $z$ direction represented the vertical distance from the nozzle to the bottom of the receiving container $(34 \mathrm{~cm}$ for the "short jet length" configuration). The noslip condition was applied along the bottom, left, and right boundaries of the domain, since these represented the solid walls of the container. Since FLOW-3D requires all three dimensions to be used in every model, the $y$ direction was chosen to be one cell deep, and symmetry conditions were applied to the front and back boundaries of the domain. Thus the 2D computational model was not actually simulating a round jet, but rather an infinitely deep planar jet.

FLOW-3D allows only one condition to be applied at each boundary, so the top of the domain was specified to have analogous fluid flowing into the domain with a uniform velocity. This velocity was chosen to match the actual nozzle velocity of the experiment being modeled (\#1 - \#6), which was calculated from the nozzle diameter and the measured mass flow rate. Solid baffles were used to block the entire top boundary except for a small gap in the center through which the fluid was allowed to enter the domain with the specified velocity. The width of this gap was chosen to match the diameter of the nozzle used in the analogous fluid experiments. This simulated the flow of analogous fluid through the nozzle into the receiving container.

Since FLOW-3D permits only a constant value for every boundary condition, the incoming analogous fluid was specified to have a constant vertical velocity. The validity of this approximation was investigated by running a $2 \mathrm{D}$ simulation in which the incoming fluid entered the domain by flowing vertically through a pipe with a width equal to the nozzle diameter, rather than simply flowing through an open section of the top boundary. This represents the actual situation in the analogous fluid experiments, where the fluid is flowing through the nozzle. Figure 4.1 shows the velocity vectors and the free surface position of $60,000 \mathrm{cSt}$ silicone oil flowing through this vertical pipe. (This figure is a close-up of a small portion of the computational domain.) The velocity is specified to be uniform as the fluid enters the pipe. The vectors at each row of cells show that the velocity profile is fully developed (parabolic) within two rows of cells $(1 \mathrm{~cm})$ from the pipe entrance. Similarly, as the fluid flows out of the pipe (the bottom of the pipe is indicated in the figure by the two large arrows), the velocity profile changes from parabolic to flat (uniform) within two rows of cells $(1 \mathrm{~cm})$ from the pipe exit. Furthermore, this simulation was run until the fluid impacted the container bottom and began to oscillate, and the frequency of oscillation was compared with another simulation in whichthe inlet pipe was removed and the velocity was specified to be uniform at the domain entrance. The two simulations were found to have the same frequency of oscillation. These results indicate that ignoring the effects of the nozzle on the velocity profile, and specifying a uniform velocity at the entrance to the domain, is a valid approximation.

Note that this set of boundary conditions does not allow any air to escape the domain, since it is enclosed on all sides. However, since the "basic" model, which is the focus of this chapter, is a single-fluid model rather than a two-fluid model, the region inside the domain is an imaginary constant-pressure void region, which has no density or mass, so it disappears when displaced by the analogous fluid entering the domain. 


\subsubsection{0,000 cSt Silicone Oil Results}

Several simulations were performed with the "basic model" with $60,000 \mathrm{cSt}$ silicone oil. Some results from a typical simulation are shown in Figures 4.2 and 4.3. Comparing these results with the photographs in Figure 1.1 demonstrates the excellent qualitative agreement between the experimental and computational results. Despite the fact that several important physical effects are not included in the "basic model," the model is clearly realistic enough to predict all the significant behaviors of the jet.

Figure 4.2(a) shows the jet beginning to neck down immediately after entering the domain. This agrees with observations of the analogous fluid experiments, in which the jets always began to neck down immediately after exiting the nozzle. Figure 4.2(b) shows the jet buckling just after impacting the bottom of the receiving container, in agreement with the experimental result shown in Figure 1.1(b). In Figure 4.2(c) the fluid is shown after the jet has oscillated through several periods and a conical pile has developed, similar to figure 1.1(d). Figure 4.2(c) also shows a void which has become entrapped in the "elbow" of the jet as it oscillates back and forth. Since this void is part of the constant-pressure region, rather than actual air, it will disappear when it is compressed as the jet swings back to the left. Figure 4.2(d) is a picture of the cone of fluid slumping and falling to the left after it has grown too tall to remain stable. This behavior is also observed in experiments, as shown in Figure 1.1(e).

Figure 4.3 is a sequence of six frames, separated by $0.01 \mathrm{sec}$, showing the jet oscillating through one period. The fluid at the buckling point swings to the left, then back to the right. In the simulations, these oscillations repeated steadily in a periodic manner. (Recall that the jet is confined to two dimensions in this "basic model," so it is actually oscillating left-to-right, rather than spiraling in a circle. In the experiments, the jet was usually observed to spiral in three dimensions, so these two-dimensional planar oscillations are not entirely realistic.)

Now that the excellent qualitative behavior of the computational model has been established, the computational results will be quantitatively compared to experimental results. The basis for this quantitative comparison will be the frequency of oscillation of the jet: the boundary conditions for the model will be chosen to correspond to the conditions of one of the experiments in Table 4.1, and the frequency of oscillation predicted by the computational model will be compared to the frequency which was measured experimentally. The computational model will first be used to simulate experiment \#2 from Table 4.1 .

Before the model's results can be compared with the experiments, it must first be shown that the results are "robust", or independent of all computational parameters such as grid resolution, time step size, etc. For example, if the frequency changes when the number of grid points is increased, the model is not robust. Four computational variables were identified as critical to the robustness of the solution.

1. and 2. Clearly, the vertical grid .... resolution (in the $z$ direction) and the horizontal grid resolution (in the $x$ direction) are two parameters which will have a strong influence on the oscillating frequency of the jet. If there are not enough grid points in the region of the domain where the jet is oscillating, the code will likely over- or under-predict the frequency. The grid resolution is especially important when using the VOF method, since a coarse grid will cause the jet to "break" (i.e., the value of $F$ will drop below 0.5 ) in the region where it oscillates back and forth. In this work, if the model predicts that the jet should break, it indicates that the solution is not correct, since the jet was never observed to break in any of the experiments.

3 . In addition to the grid resolution in the $x$ and $z$ directions, a third parameter, which has an effect on the solution's 
robustness, is the FLOW-3D variable ITVSMX. This variable controls the maximum number of viscous stress iterations allowed for each time step. In FLOW-3D, the user has the option of calculating the viscous stress implicitly or explicitly. Since the fluids considered in this work have such high viscosities, the time step required for stability in the explicit calculation of the viscous stress is three orders of magnitude smaller than the time steps required for stability by the other terms in the equation, so an explicit calculation of this stress would increase the time required to obtain a solution by three orders of magnitude. This is clearly unacceptable, so it was decided to evaluate the viscous stress implicitly rather than explicitly. An implicit evaluation does not place a limitation on the time step size, but it requires the viscous stress at each time step to be calculated iteratively. For the cases considered in this work, the solver always uses the maximum number of viscous stress iterations that are allowed for each time step after the jet impacts the container, so it is expected that increasing the value of ITVSMX will lead to a more accurate solution, since the increased number of ..... iterations will allow the viscous stress to be calculated more accurately.

4. The fourth parameter that was considered to affect the robustness of the solution was EPSADJ, the factor that FLOW$3 \mathrm{D}$ uses to adjust the convergence criterion for a time step. As explained in Section 2.3.1, FLOW-3D calculates the time step size based on the values of several non-dimensional stability criteria. These criteria are also used to determine the recommended value of $\varepsilon$, the convergence criterion for the iterative calculation of the pressure. If the user feels that the value of $\varepsilon$ recommended by FLOW$3 \mathrm{D}$ is not appropriate, this value may be adjusted by specifying EPSADJ. If a value is specified for EPSADJ, then FLOW-3D multiplies its recommended value of $\varepsilon$ by EPSADJ to determine the actual convergence criterion used. For example, if EPSADJ = 0.1 , this makes the convergence criterion "tighter" by one order of magnitude.

To determine if the solution was robust, a "base case"- model was solved first, with arbitrarily chosen values for the $z$ grid resolution, $x$ grid resolution, ITVSMX, and EPSADJ. (The $z$ and $x$ grid resolutions were quantified by defining them to be the number of horizontal rows of grid points in the bottom $40 \%$ of the domain and the number of vertical columns of grid points in the middle $1 / 3$ of the domain, respectively. These definitions were chosen because the oscillation of the jet occurs in the horizontal center of the domain near the vertical bottom, so the grid in this region will play the most significant role in the prediction of the frequency.) The "base case" model had 48 horizontal rows of grid points in the bottom $40 \%$ of the domain, 23 vertical columns of grid points in the middle $1 / 3$ of the domain, ITVSMX $=500$, and EPSADJ $=0.1$. Each of the four variables was then varied independently, and the effect on the frequency of oscillation was observed.

The first variable whose effect was considered was the $z$ grid resolution. The number of horizontal rows of grid points in-.. the bottom $40 \%$ of the domain was varied from 12 to 80 , while holding the $x$ grid resolution, ITVSMX, and EPSADJ fixed at the values stated in the previous paragraph. Figure 4.4 shows the frequency of oscillation as a function of the $z$ grid resolution. Over the range of resolutions considered, the frequency changes by a factor of three, indicating that the frequency is quite sensitive to the $z$ grid resolution. Figure 4.4 indicates that the frequency of oscillation approaches a fixed value as the resolution is increased. This can be seen from the shape of the frequency-vs.-resolution curve, which flattens out as the resolution is increased, showing that successive increases in the resolution have less and less impact on the frequency. When this curve is flat, the frequency is independent of the value of the $z$ grid resolution (any further increase in the 
resolution will not change the frequency), and a robust solution has been achieved. Since the calculated frequency changes by only $0.7 \%$ between the two finest $z$ grid resolutions attempted; the solution is nearly independent of the $z$ grid resolution for any value of $z$ grid resolution above 64 .

The effect of the $x$ grid resolution was considered next. The number of vertical columns of grid points in the middle $1 / 3$ of the domain was varied from 15 to 23 while holding the other three variables constant. Figure 4.5 is similar to Figure 4.4 , but it shows the frequency of oscillation as a function of $x$ grid resolution rather than $z$ grid resolution. Like Figure 4.4, it shows that the frequency is approaching a fixed value as the $x$ grid resolution is increased, indicating that the solution is approaching robustness. The calculated frequency changes by only $2 \%$ between the two finest $x$ grid resolutions attempted, which shows that the solution is fairly independent of the $x$ grid resolution for any value of $x$ grid resolution above 19. Less data points appear on Figure 4.5 than on" Figure 4.4 because the percent change in frequency was relatively low for all $x$ grid resolutions attempted, so the solution was fairly robust for even the coarsest grid.

Figure 4.6 shows the frequency of oscillation and percent change in frequency as a function of the value of ITVSMX. Like the previous two figures, Figure 4.6 shows that the frequency approaches a constant value as ITVSMX is increased: again, the solution is tending toward robustness. Since the calculated frequency changes by only $0.7 \%$...... between the two largest values of ITVSMX attempted, the solution is practically independent for any value of ITVSMX above 500.

Finally, the value of EPSADJ was varied while holding the other three variables constant. For EPSADJ $=0.1$ (the "base case") and $E P S A D J=1.0$, the frequency was identical. This indicates that the default value of $\varepsilon$ which is automatically selected by
FLOW-3D is appropriate, and does not need to be adjusted by the user.

In summary, the four variables that were expected to affect the jet's frequency of oscillation most significantly were each varied independently over a range of values. As each variable was changed in a way that would be expected to lead to a more accurate result, the frequency approached a constant value. This shows that the solution is in fact robust, when each of the four variables are chosen to be in the range where the frequency is unchanging.

Of the several solutions plotted in Figures $4.4-4.6$, the one that is believed to be the most accurate is the rightmost data point on Figure 4.4. This solution was obtained with 80 horizontal rows of grid points in the bottom $40 \%$ of the domain, 23 vertical columns of grid points in the middle $1 / 3$ of the domain, ITVSMX $=500$, and EPSADJ = 0.1 . This solution is considered to be the most accurate because it uses the most robust value of $z$ grid resolution, $x$ grid resolution, and EPSADJ, and it uses the second-most robust value of ITVSMX (which predicted a frequency that was only $0.7 \%$ different from the most robust ITVSMX solution on Figure. 4.6).

The frequency of oscillation calculated when using these parameters is $15.2 \mathrm{~Hz}$. Recall that the boundary conditions were chosen to simulate experiment \#2, which had experimentally measured frequencies between 12.6 and $13.2 \mathrm{~Hz}$. The discrepancy between the experimental and computational results can be attributed to the fact that there was no. initial pool height in the computational simulation - the jet flowed into the container, hit the bottom, and began to oscillate and fill the container, and then the frequency was measured while the oscillating portion of the jet was still fairly close to the bottom of the container. In other words, the jet length in the computational model was longer than the jet length in the experiment. Since the frequency of the oscillations decreases as the container fills up, a result that has been observed both 
computationally and experimentally, it is believed that the difference in pool height between the computational and experimental cases is the primary cause of the discrepancy between the computational and experimental frequencies.

To confirm this hypothesis, the videotape of experiment \#2 was reviewed, and several frequency values were measured during the eight seconds of the experiment. Using these data, a correlation between pool height and spiraling frequency was obtained. This correlation was extrapolated backward to approximate the spiraling frequency for a pool height of zero, giving a value of $15.7 \mathrm{~Hz}$. The accuracy of this value is questionable, since the pool height of zero is far outside the range of pool heights used to generate the correlation $(4.8-6.1 \mathrm{~cm})$. Nonetheless, the oscillation frequency in the FLOW-3D model differs from this value by only $3 \%$, indicating excellent quantitative agreement between the computational model and the experimental behavior of the jet.

\subsubsection{2,500 cSt Silicone Oil Results}

To evaluate the performance of

- FLOW-3D when modeling a fluid other than $60,000 \mathrm{cSt}$ silicone oil, simulations were performed with the "small jet length" configuration and the properties of $12,500 \mathrm{cSt}$ silicone oil. The boundary conditions in these simulations were specified to match the conditions of experiment \#6 in table 4.1. Unlike the simulations described in the previous section, these simulations included an initial condition specifying the pool height, so the incoming fluid jet impinged upon the pool free surface rather than the solid bottom of the container as in the previous section. This matches the actual experimental conditions, in which the container was filled up to a certain pool height, the jet was stopped and the pool surface was allowed to flatten out, and then the motor was turned on again and the jet flowed into the pool of stagnant fluid.
Figure 4.7(a) shows the flow after the jet has impinged upon the free surface, buckled, and begun to oscillate. Comparing this result with Figure 4.7(b), which shows the 60,000 cSt silicone oil from experiment $\# 2$ that was discussed in the previous section, emphasizes several differences between the two flows. (Note that the scale in Figures 4.7(a) and (b) is the same, with identical container and nozzle dimensions.) The necking of the 12,500 cSt silicone oil jet is much more pronounced than that of the $60,000 \mathrm{cSt}$ silicone oil jet, and therefore the diameter of the $12,500 \mathrm{cSt}$ silicone oil jet is smaller in the buckling region. This is largely due'to the difference in mass flow rates between the two flows: the mass flow rate of the $12,500 \mathrm{cSt}$ silicone oil is $1 / 3$ less than that of the $60,000 \mathrm{cSt}$ silicone oil (as seen in Table 4.1). Another difference is that the amplitude of the oscillations of the $12,500 \mathrm{cSt}$ silicone oil jet is much smaller than the amplitude of the $60,000 \mathrm{cSt}$ silicone oil jet's oscillations. This agrees with experimental results, where it was observed that smaller-diameter jets tended to spiral with a smaller amplitude. Another result from the FLOW-3D models, which agrees with experiments, is that jets with smaller diameter have a higher spiral frequency. The $12,500 \mathrm{cSt}$ silicone oil jet shown in Figure 4.7(a) had a frequency of oscillation of $44.2 \mathrm{~Hz}$, whereas the $60,000 \mathrm{cSt}$ silicone oil jet had a frequency of only 15.2 $\mathrm{Hz}$. The difference in height of the conical piles formed by the oscillating jets, with the higher mass flow rate jet forming a taller pile, also agrees with the behavior observed in the experiments. The most striking difference between Figures 4.7(a) and (b) is the shape of the pool's free surface in the two flows. The $12,500 \mathrm{cSt}$ silicone oil jet significantly depresses the surface of the pool in the landing region, but the $60,000 \mathrm{cSt}$ silicone oil jet does not disturb the flat surface of the pool, forming a cone that sits on top of the surface. This is a surprising result, and one which may appear to be erroneous; however, this is exactly the behavior which was 
observed in the experiments, where jets of $60,000 \mathrm{cSt}$ silicone oil always piled up on top of the surface, but jets of $12,500 \mathrm{cSt}$ silicone oil tended to depress the free surface in the landing region. This different behavior is due to the difference in the viscosity of the two fluids, since all other parameters and fluid properties are identical.

Due to time constraints, it was not possible to thoroughly evaluate the robustness of the 12,500 cSt silicone oil model as was done for the $60,000 \mathrm{cSt}$ silicone oil model. Only two simulations of the $12,500 \mathrm{cSt}$ silicone oil jet flow were performed. The first simulation had 38 horizontal rows of grid points and 23 vertical columns of grid points in the buckling region, ITVSMX $=300$, and EPSADJ $=0.1$. This model predicted a frequency of oscillation of $41.1 \mathrm{~Hz}$. However, the jet "broke" frequently while oscillating, i.e. some of the computational cells within the jet's path had values of $F<$ 0.5 . Since the jet was never observed to break during the experiments, this breaking was caused by insufficient grid resolution, so the horizontal grid resolution was increased to eliminate the jet breakage. With the increased grid resolution, the jet oscillated at a frequency of $44.2 \mathrm{~Hz}$, and did not break. (The result shown in Figure 4.7(a) was obtained from this model.) This result, while qualitatively reasonable, does not compare well with the experimentally measured frequency of $29.4 \mathrm{~Hz}$. While the robustness of this FLOW-3D model has not been verified, it is believed that the frequency predicted by a fully robust model would not be significantly different from the frequency predicted by the current model, since the values of all the significant computational parameters $(z$ and $x$ grid resolution, ITVSMX, and EPSADJ) are near or within the robust range determined for the $60,000 \mathrm{cSt}$ silicone oil jet. The discrepancy between the FLOW3D model's frequency of oscillation and the experimentally measured spiral frequency may be attributed to a difference in the jet diameter in the landing region. The only qualitative difference between the FLOW-3D $12,500 \mathrm{cSt}$ silicone oil model results and the experimental jet behavior is that the jet in the model necks more than the real jet in the experiment, so the FLOW-3D model's jet has a smaller diameter in the landing region, which would increase the frequency of oscillation of the jet, as is observed here. It is not known why the computational model over-predicts the necking of the $12,500 \mathrm{cSt}$ silicone oil jet.

\subsubsection{Problems}

Despite the good agreement between the computational model results and the experimentally measured results, there were a few problems with FLOW-3D's implementation of the model that will be briefly discussed here.

One of these problems concerns the implicit viscous stress calculations. As mentioned in Section 4.3.2, this calculation is performed iteratively and repeated until a convergence criterion is satisfied, and the variable ITVSMX is used to control the maximum number of iterations allowed per time step. However, it was observed in all simulations that after the jet impacted the bottom of the container and began to buckle and oscillate, the solver always used the maximum allowable number of iterations (ITVSMX) for every time step. This suggests that the viscous stress was never satisfying the convergence criterion, which would be expected to introduce an unacceptable level of error into the calculation. A series of simulations was performed in which the value of ITVSMX was increased up to 5000, and the solver still always used the maximum allowable number of iterations for every time step. Ideally, the viscous stress would satisfy the convergence criterion at every time step, but it is impractical to use values of ITVSMX greater than 1000 since it takes too long to obtain a solution. Therefore, the value of ITVSMX was considered as one of the variables which significantly affects the solution, and its value was increased until the 
solution was found to be robust (see Figure 4.6). When the value of ITVSMX is chosen to be in the "robust region" on the right-hand end of the curve in Figure 4.6, further increases in ITVSMX do not affect the solution. Thus, although the FLOW-3D solver does not consider the viscous stress to be converged, further iterations have no practical effect on the solution, and so the solution may be considered to be robust with respect to the viscous stress.

A second problem with the performance of FLOW-3D was observed when the viscous stress was evaluated explicitly rather than implicitly. In this case, two problems arose. First, in all simulations where the viscous stress was calculated explicitly, after the jet impacted the bottom of the container, the pressure in the pool was observed to increase suddenly by two or three orders of magnitude from one time step to the next. Also, regions of extremely high and extremely low pressure developed in the fluid pool, and these regions would occasionally switch places (the high-pressure region would suddenly drop to a very low pressure, and vice versa) from one time step to the next. These results, which were not physically realistic, were not observed when the viscous stress was evaluated implicitly. It is believed that this pressure problem can be eliminated by setting the value of the FLOW-3D variable OMEGA equal to one; however, this has not been thoroughly investigated, and it may not work for all cases. (OMEGA controls the over-relaxation used in the pressure iterations, so setting it equal to one would be expected to make the pressure calculations more stable than when using the default value of 1.7.) The second problem which was encountered when the viscous stress was evaluated explicitly was that the jet either did not buckle at all after impacting the container, or buckled very slowly. Figure 4.8 shows a comparison between the jet shape when the viscous stress was calculated explicitly and implicitly (with all other computational parameters the same) for a typical simulation. Since the jet was observed to buckle and spiral in all of the experiments, the result obtained when the viscous stress was calculated implicitly (Figure 4.8(b)) is clearly much more realistic than the result when the viscous stress was calculated explicitly (Figure 4.8(a)). Because of this problem, as well as the erroneous pressure results, it was decided to calculate the viscous stress implicitly, despite the problem with ITVSMX mentioned in the previous paragraph.

The third and final problem with the FLOW-3D calculations for the "basic model" of this chapter was that in some cases, the jet was observed to swing to the right or left in the early stages of the calculation as it was falling toward the container bottom, rather than falling straight down. Of course there is no physical reason why the jet would swing to the side, so this is clearly a computational error. It was found that increasing the grid resolution in the region where the jet was falling eliminated this problem.

\subsection{FIDAP}

\subsubsection{Boundary and Initial Conditions}

In FIDAP, as in FLOW-3D, a

rectangular Cartesian grid was used in the "basic model." The domain in the $x$ direction represented the width of the receiving container, and the domain in the $z$ direction represented the distance from the nozzle to the bottom of the receiving container. The noslip condition was applied along the bottom, left, and right boundaries of the domain, since these represented the solid walls of the container. Unlike FLOW-3D, FIDAP allows the model to be specified as two-dimensional, so there were no conditions required for the front or back boundaries.

FIDAP allows multiple conditions to be specified along any boundary, so two conditions were specified along the top boundary. Along part of the top boundary, in a region with a width equal to the nozzle used in the analogous fluid experiments, it was specified that the analogous fluid was flowing 
into the domain with a uniform velocity which was chosen to match one of the six experiments in Table 4.1. Along the rest of the top boundary, it was specified that the velocity flux was equal to zero. This is the default boundary condition, and it allows fluid to flow freely into or out of the domain. Thus all of the FIDAP boundary conditions are the same as the FLOW-3D boundary conditions, except for the condition along the open portion of the top boundary. Since this "basic model" simulates only a single fluid, there is no air in the domain to be displaced by the incoming jet, so the boundary condition along the open portion of the top boundary should not affect the solution, and the two codes are expected to produce the same solution.

A special set of initial conditions was required when solving the problem with FIDAP. The FIDAP implementation of the VOF method requires that at least a small amount of fluid must be present in the domain at all times. Otherwise, in the first time step when a tiny amount of fluid has flowed into the domain, the solver is unable to calculate correct values for the fluid velocity. For this reason, in all FIDAP simulations, a small slug of fluid was specified to be initially directly below the jet inlet. This slug had an initial uniform velocity equal to the velocity of the incoming fluid jet. This simulated the condition of the jet after a short time had passed, when a small amount of fluid had flowed into the domain.

\subsubsection{Results}

Typical results obtained when using

FIDAP to solve the small jet length problem with $60,000 \mathrm{cSt}$ silicone oil are displayed in Figure 4.9. A cursory comparison of this figure with the experimental results in Figure 1.1 shows that the FIDAP solution does not agree qualitatively with the real jet behavior, and comparing Figures 4.9 and 4.2 demonstrates that the FIDAP computational results do not match the FLOW-3D computational results. (When comparing Figures 4.2 and 4.9, keep in mind that the dimensions of the container, the nozzle width, the jet inlet velocity, and the vertical and horizontal grid resolution are identical in the two figures.)

Figure 4.9(a), which shows the jet just before it impacts the bottom of the container, indicates that the jet does not neck down at all as it flows into the domain. This is in contrast to Figure 4.2(a), the FLOW-3D result, which predicts that the jet begins to neck down immediately upon entering the domain. Since the two codes have the same boundary condition applied at the nozzle region (uniform vertical velocity), they would be expected to produce the same result, but this is clearly not the case. In the real experiments, the jets always began to neck down immediately after exiting the nozzle, which disagrees with the FIDAP result.

Another disparity between the FIDAP result and the real behavior of the jet is shown in Figure 4.9(b). In FIDAP, after the jet impacts the bottom of the container, it does not buckle immediately, but rather it remains straight and grows thicker. This behavior was never observed in any of the analogous fluid experiments; on the contrary, as predicted by the FLOW-3D solution (Figure 4.2(b)), ,the jet is expected to buckle immediately after -... impacting the container.

When the FIDAP jet finally does buckle, as shown in Figure 4.9(c), it buckles a great distance from the bottom of the container, and the oscillations have a very large amplitude. As with the previous two FIDAP plots, this type of jet behavior was never observed experimentally. Instead, the jet buckled near the bottom of the container, as in the FLOW-3D result shown in Figure 4.2(b).

Several computational parameters were varied to see if they were the cause of the unrealistic solution. The grid resolution was doubled in both the $x$ and $z$ directions. The problem was solved with quadratic basis functions rather than linear. The effects of surface tension were included in some simulations, and not included in others. A 
section of vertical pipe was added to the top of the domain, to see if the initial slug of jet fluid was the cause of the problems.

However, none of these changes had any effect on the solution - the jet continued to behave as shown in Figure 4.9. Thus it is not known why FIDAP generates these unrealistic results when simulating the "small jet length" flow.

Table 4.1: Results of Small Jet Length Experiments

$\begin{array}{ccccc}\begin{array}{c}\text { Experiment } \\ \text { Number }\end{array} & \begin{array}{c}\text { Silicone oil } \\ \text { viscosity }(\mathrm{cSt})\end{array} & \begin{array}{c}\text { Pool } \\ \text { height }(\mathrm{cm})\end{array} & \begin{array}{c}\text { Mass flow } \\ \text { rate }(\mathrm{g} / \mathrm{s})\end{array} & \begin{array}{c}\text { Frequency } \\ (\mathrm{Hz})\end{array} \\ 1 & 60,000 & 2.0 & 8.8 \pm 0.007 & 16.7 \pm 0.09 \\ 2 \mathrm{a} & 60,000 & 4.8 & , 36.5 \pm 0.06 & 13.2 \pm 0.05 \\ 2 \mathrm{~b} & 60,000 & 6.1 & 36.5 \pm 0.06 & 12.6 \pm 0.05 \\ 3 & 12,500 & 2.6 & 3.5 \pm 0.003 & 87.4 \pm 0.7 \\ 4 & 12,500 & 3.8 & 10.2 \pm 0.06 & 56.6 \pm 0.07 \\ 5 & 12,500 & 6.1 & 16.5 \pm 0.04 & 42.1 \pm 0.03 \\ 6 & 12,500 & 9.7 & 24.8 \pm 0.06 & 29.4 \pm 0.08\end{array}$




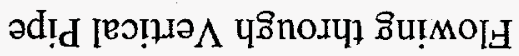

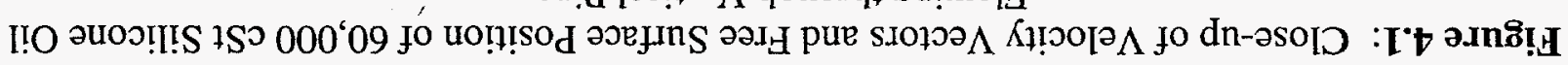

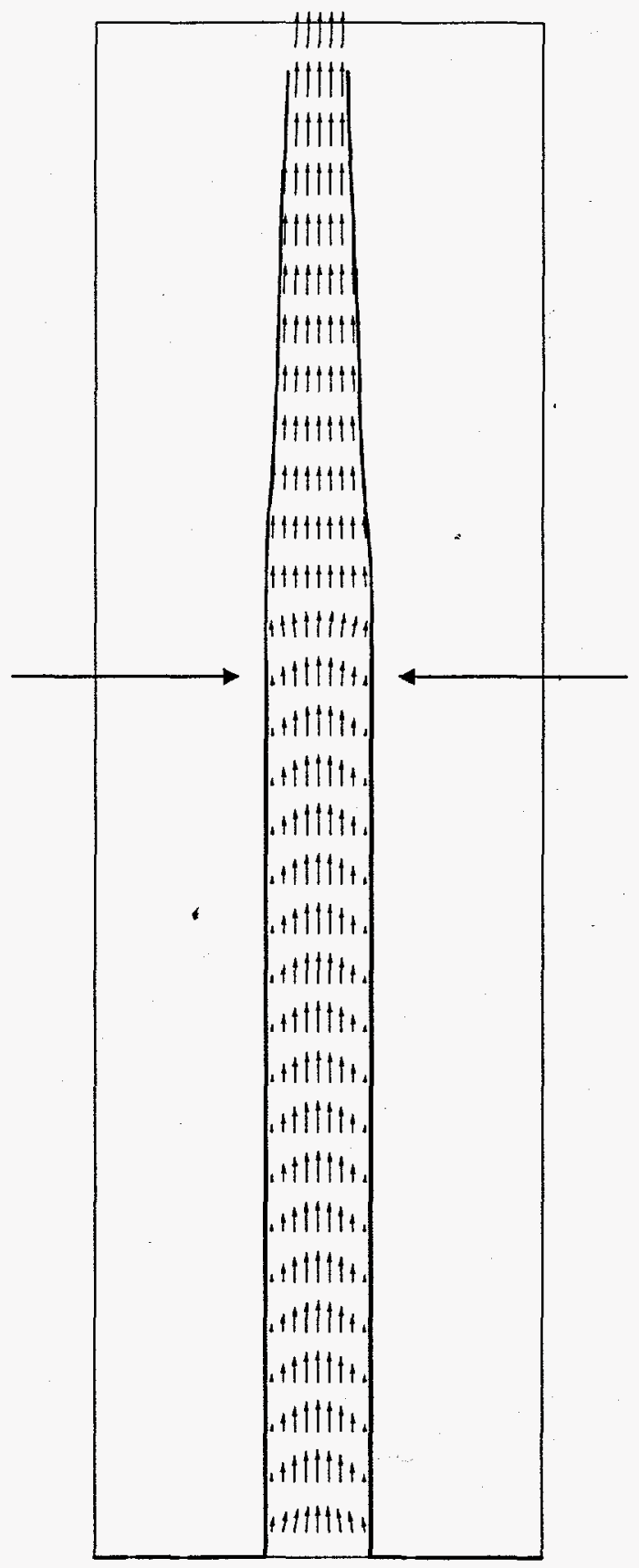




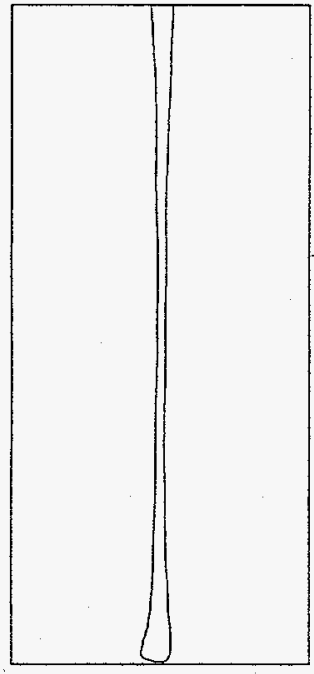

(a)

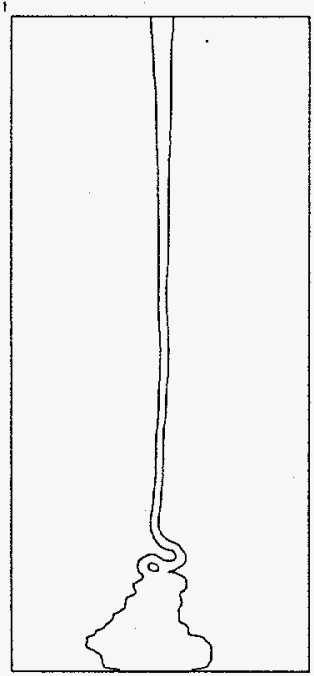

(c)

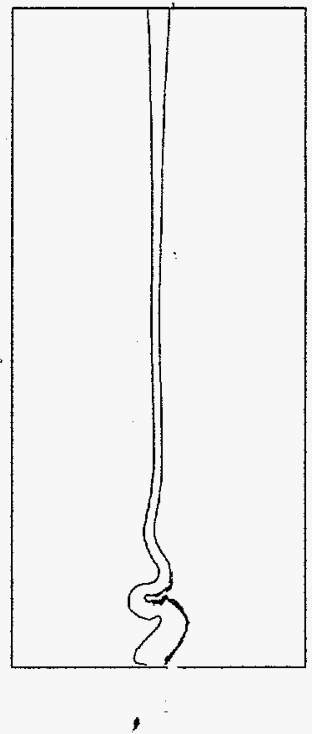

(b)

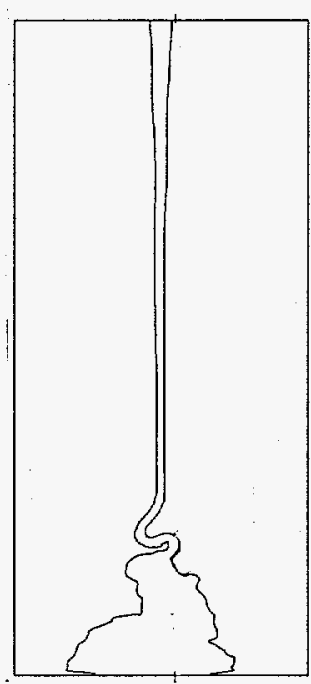

(d)

Figure 4.2: Typical FLOW-3D Results for $60,000 \mathrm{cSt}$ Silicone Oil with Short Jet Length. The solid line represents the free surface of the fluid. (a) Just before impact. (b) Initial buckling. (c) Oscillating and entrapping a void. (d) Cone slumping to left. 


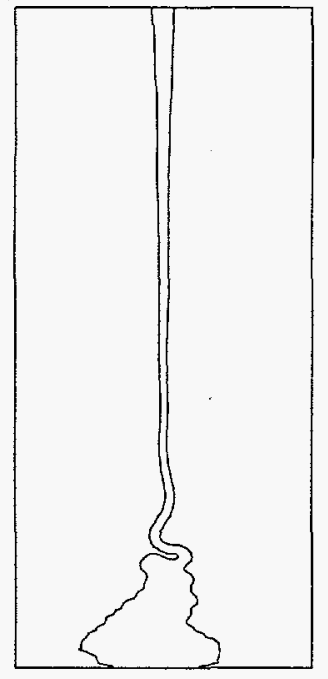

(a)

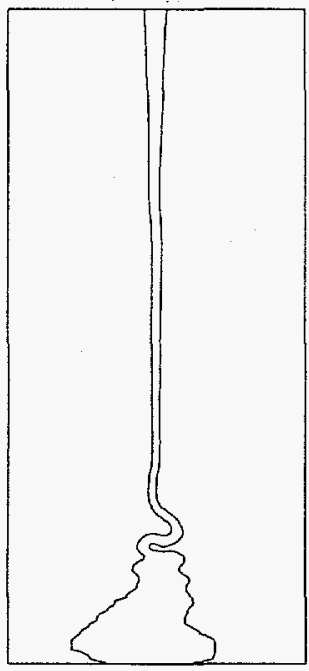

(d)

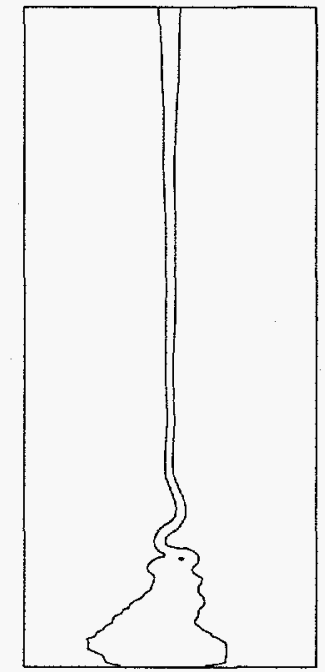

(b)

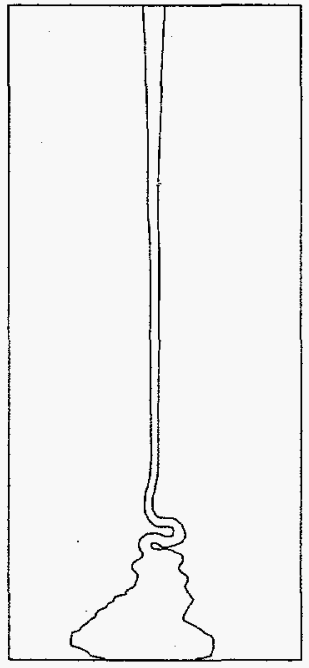

(e)

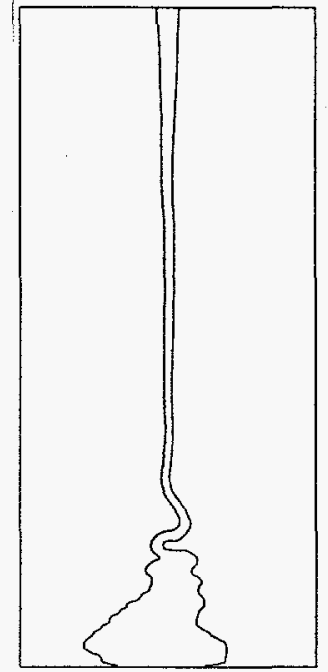

(c)

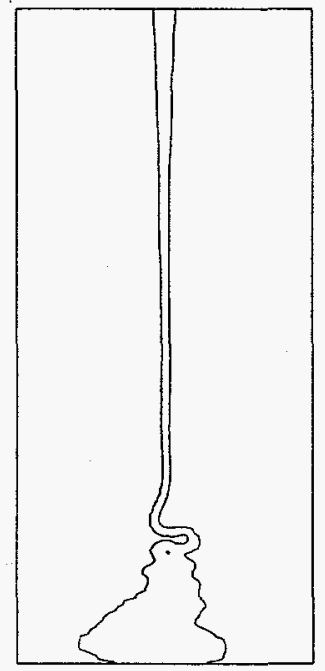

(f)

Figure 4.3: Sequence of Six Consecutive Frames Showing One Period of Jet Oscillation for a Typical $60,000 \mathrm{cSt}$ Silicone Oil Jet with Short Jet Length. Frames are separated by $0.01 \mathrm{sec}$. 


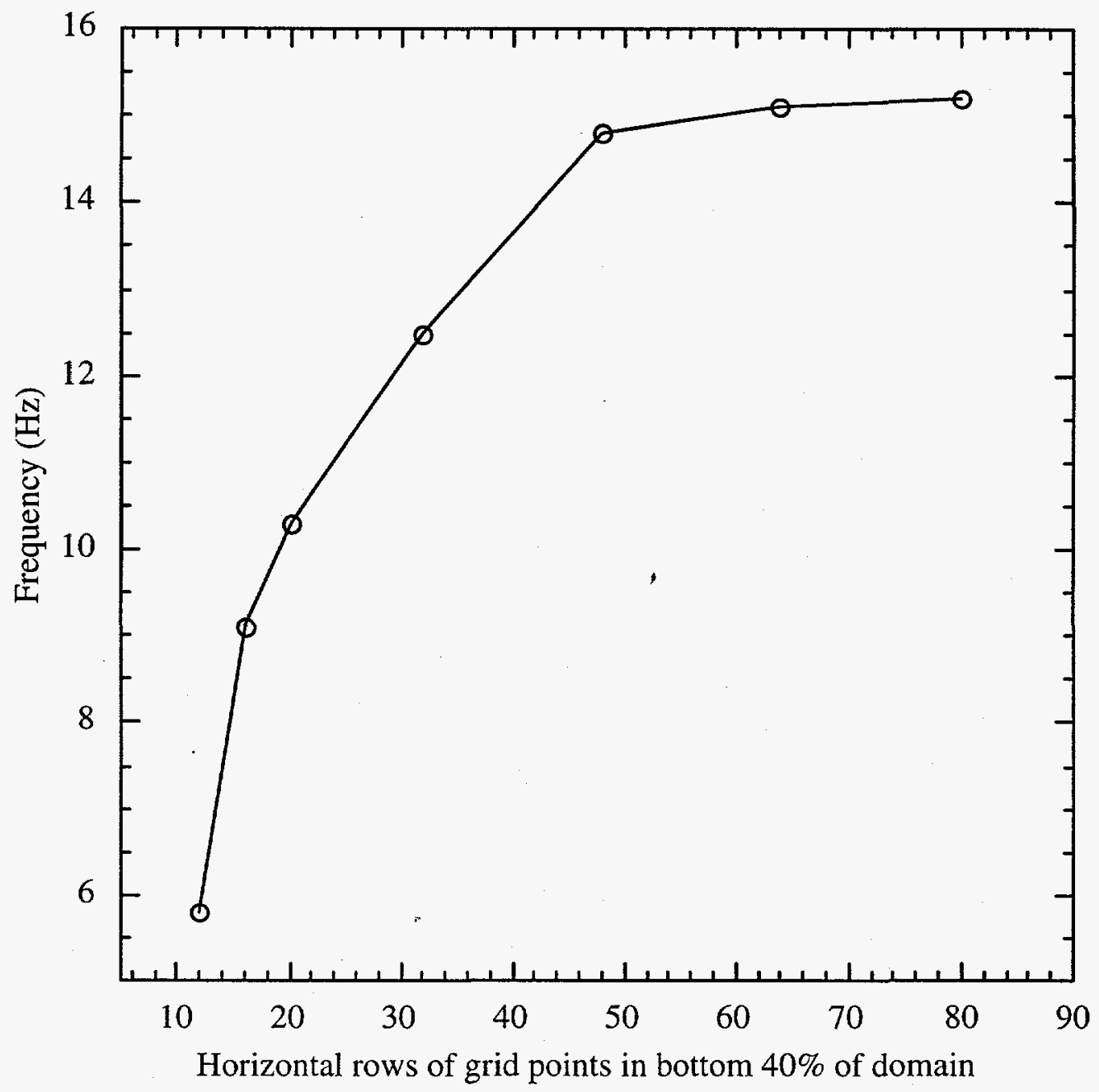

Figure 4.4: Effect of Vertical Grid Resolution on Frequency of Jet Oscillation 


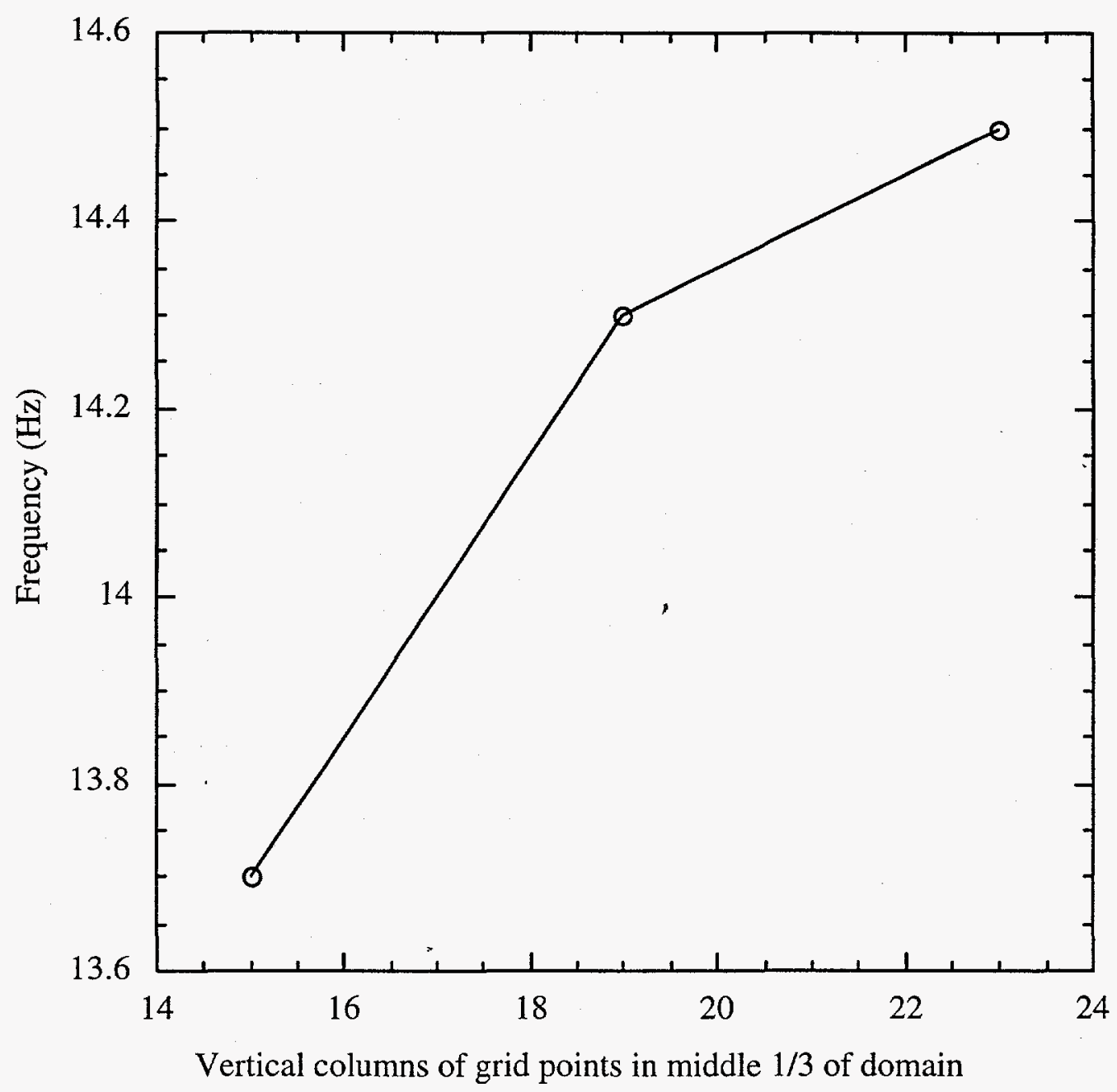

Figure 4.5: Effect of Horizontal Grid Resolution on Frequency of Jet Oscillation 


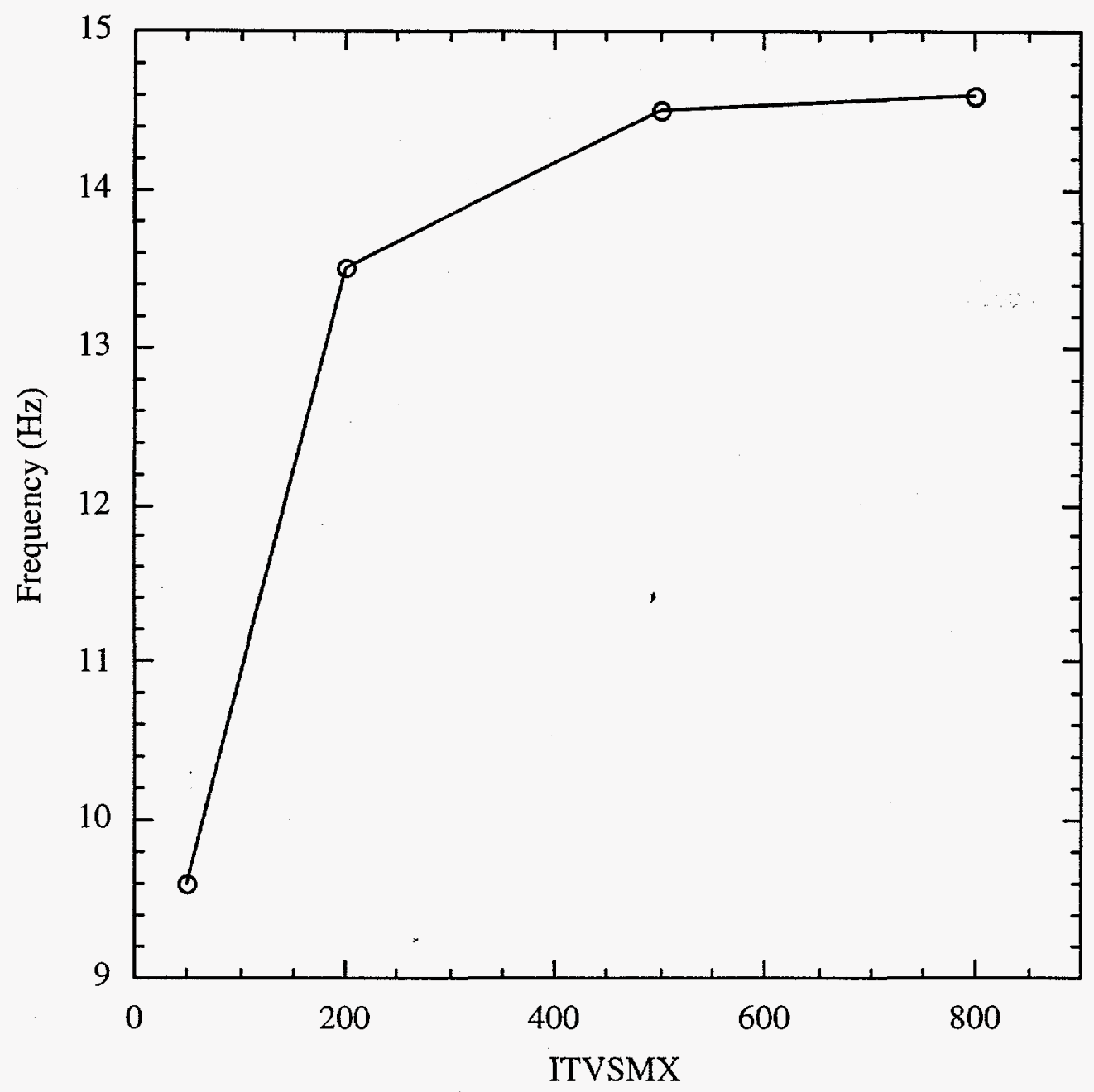

Figure 4.6: Effect of ITVSMX on Frequency of Jet Oscillation 


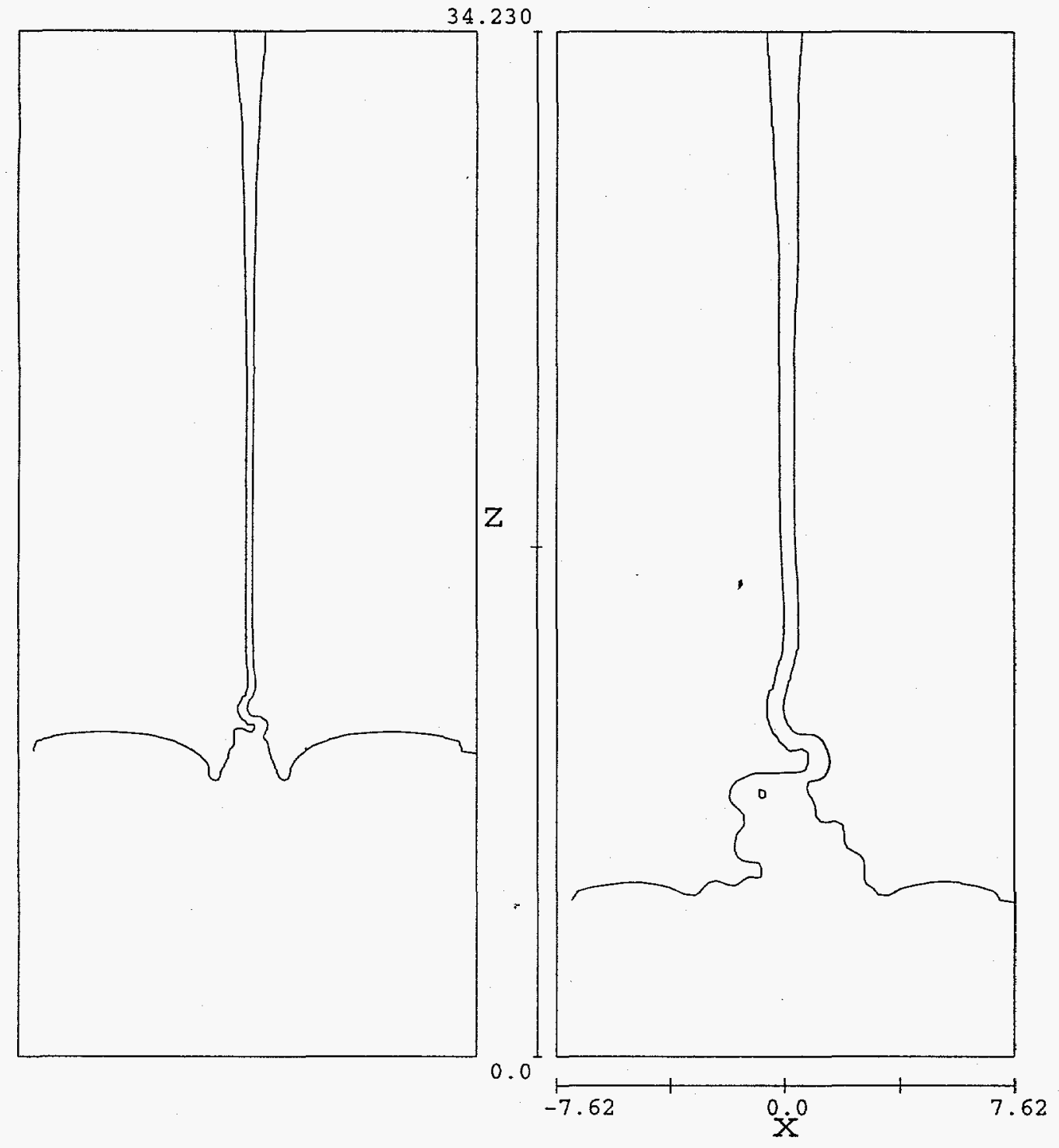

(a)

(b)

Figure 4.7: Free Surface Behavior for Different Fluids.

(a) $12,500 \mathrm{cSt}$ silicone oil, $24.8 \mathrm{~g} / \mathrm{s}$. (b) $60,000 \mathrm{cSt}$ silicone oil, $36.5 \mathrm{~g} / \mathrm{s}$. 


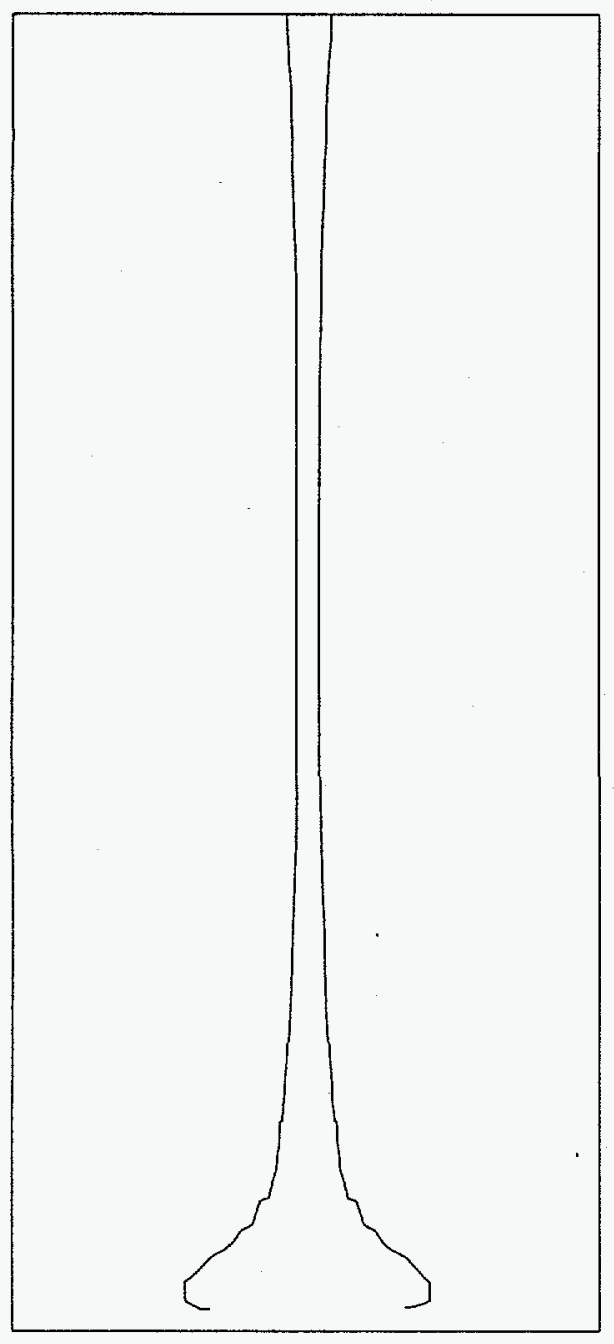

(a)

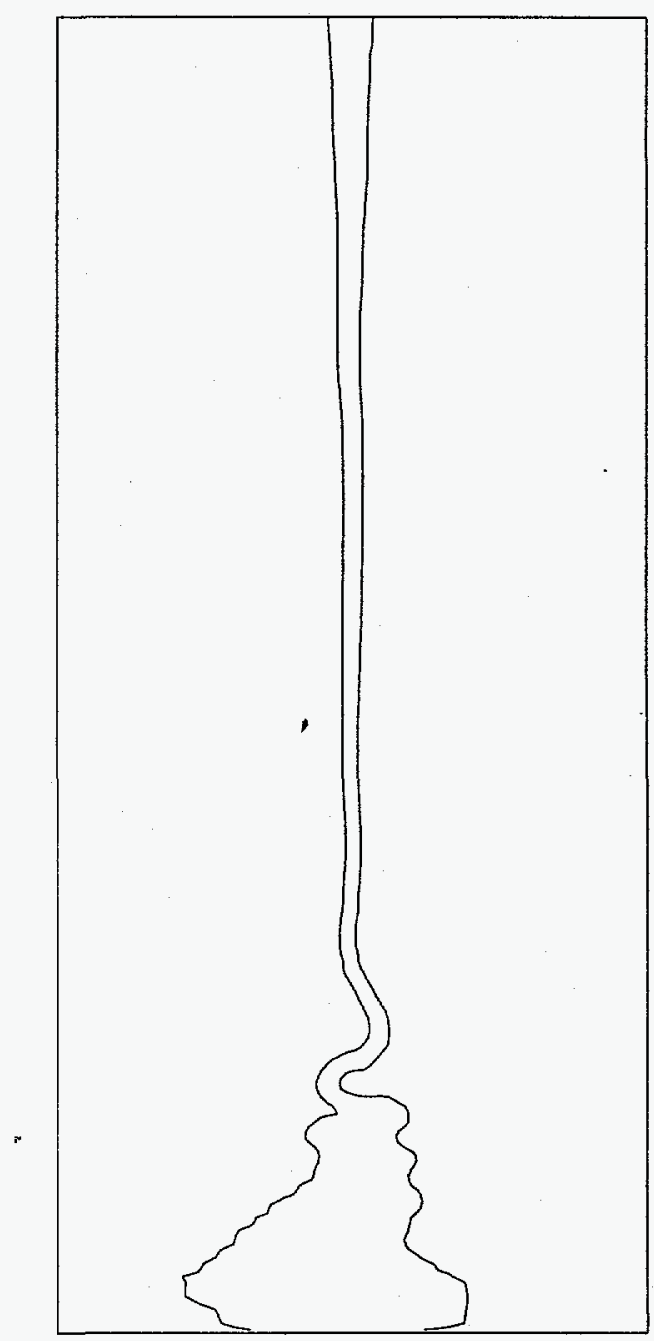

(b)

Figure 4.8: (a) Typical FLOW-3D Result with Viscous Stress Calculated Explicitly (b) Same Case, with Viscous Stress Calculated Implicitly 


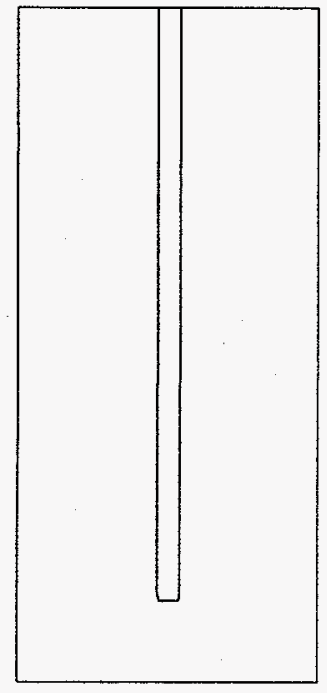

(a)

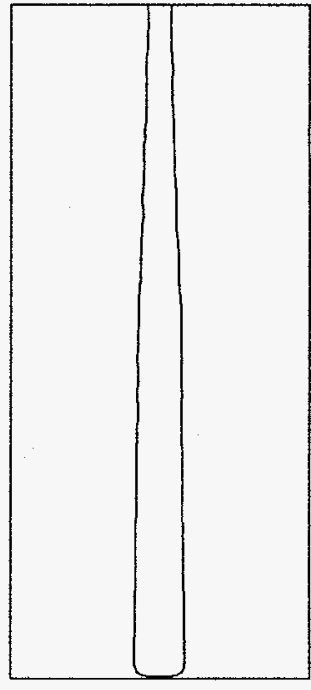

(b)

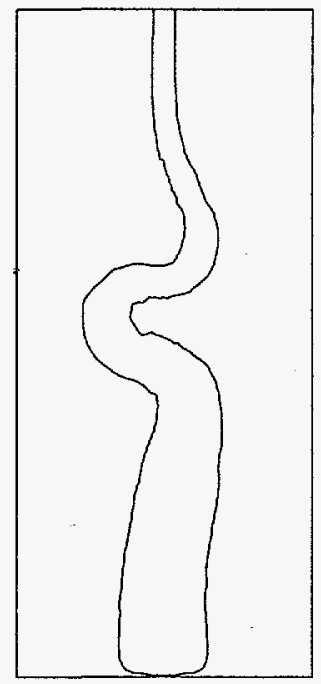

(c)

Figure 4.9: Typical FIDAP Results for 60,000 cSt Silicone Oil with Short Jet Length (a) Just before impact. (b) Jet thickening. (c) Initial buckling. 
, 


\section{THREE-DIMENSIONAL MODEL}

\subsection{DESCRIPTION OF COMPUTATIONAL MODEL}

This chapter will focus on the "3D model." This model is similar to the basic model, which was discussed in Chapter 4, but instead of being limited to two dimensions, the fluid simulated with the 3D model will be permitted to flow in all three spatial dimensions. The 3D model has the following characteristics:

- three-dimensional,

- small jet length,

- single fluid, and

- isothermal.

\subsection{FLOW-3D}

\subsubsection{Boundary Conditions}

Like the basic model, the 3D model used a Cartesian grid system. The domain in the $x$ and $y$ directions represented the width and depth of the receiving container, which were equal, and the domain in the $z$ direction represented the distance from the nozzle to the bottom of the receiving container, which was $34 \mathrm{~cm}$ for the "small jet length" configuration. The no-slip condition was applied along the bottom, left, right, front, and back boundaries of the domain, since these represented the solid walls of the container. The top boundary was specified to have a uniform velocity of fluid flowing into the domain, and a solid baffle was used to block the entire boundary except for a circular hole in the center through which the fluid was allowed to enter the domain. The diameter of this hole was chosen to match the diameter of the nozzle used in the analogous fluid experiments. This simulated the flow of analogous fluid through the nozzle into the receiving container.

\subsubsection{Results}

Of course, the addition of the third spatial dimension greatly increased the time required to obtain a solution. Because of time limitations, only one 3D simulation could be performed. A result from this simulation is shown in Figure 5.1, demonstrating that the 3D model, like the basic model, qualitatively matches the real jet behavior. Figure 5.1 shows the jet spiraling in a circle and forming a tall column of fluid, similar to the experimental result shown in Figure 1.1(d). The necking of the jet is also shown in Figure 5.1 .

To provide data for a comparison, a $2 \mathrm{D}$ rnodel was developed which had the same $x$ and $z$ grid resolution as the 3D model. When the spiraling frequency of the 3D jet was compared with the frequency of oscillation of the 2D jet, it was found that both models predicted exactly the same frequency for early times in the simulation, soon after the jet impacted the container. Therefore, for purposes of predicting the jet frequency, the 2D model is just as accurate as the $3 \mathrm{D}$ model. This is a very significant result, because the use of a 2D model instead of a 3D model provides a tremendous savings in the time required to obtain a solution.

However, for later times in the simulation, after the jet had begun to pile up and fill the container, the 2D model predicted lower values of the frequency than the 3D model. This is due to the difference in the effective fill rate of the two models. If the 2D and 3D models have the same inlet velocity, nozzle diameter, and container width, the 2D domain will effectively fill up 16 times as fast as the $3 \mathrm{D}$ domain. In the analogous fluid experiments, the width (and length) of the container is $15.24 \mathrm{~cm}$, and the nozzle diameter is $1.18 \mathrm{~cm}$. If the inlet velocity is $34.89 \mathrm{~cm} / \mathrm{s}$, then after one second, $7.8 \%$ of the volume in the 2D domain will be filled with fluid, but only $0.5 \%$ of the volume in the 3D domain will be filled. As the 2D domain effectively fills faster, the jet length decreases more rapidly, causing the frequency of oscillation 
to decrease. This effect is demonstrated in Figure 5.2, which shows the $2 \mathrm{D}$ result and a plane slice through the center of the 3D result for two different times. At an early time in the simulation (Figures 5.2(a) and 5.2(b)), the point in the jet where the buckling occurs is about the same for the 2D and 3D models, but at a later time (Figures 5.2(c) and 5.2(d)), the buckling location in the 2D jet is higher than in the 3D jet. (The 3D jet appears to have broken because the plots in Figures 5.2(b) and (d) show only a slice through the midplane of the jet. However, inspection of Figure 5.1 will show that the jet did not break.) Therefore, when predicting the frequency of oscillation of the jet with the 2D model, one must take care to match the pool height of the experiment rather than the elapsed time.

Besides the different fill rate between the $2 \mathrm{D}$ and $3 \mathrm{D}$ models, two other differences were observed. The first is the mechanism by which voids become entrapped in the fluid pool. As shown in Figure 4.2(c), the 2D jet entraps voids by "pinching" them in the "elbow" of the jet as it oscillates from side to side. In the 3D model, this side-to-side motion is replaced by a circular spiraling motion, and it seems unlikely that voids would become entrapped by this motion, since there is no "elbow" to "pinch" and entrap a void. However, as shown in Figure 5.1 , the $3 \mathrm{D}$ jet forms a tall, hollow cylindrical column with air-at its center. As this column becomes unstable and slumps to the side, or as the erratic spiraling motion closes off the top of this column, the air at the center of the column will become entrapped in the fluid pool. The second difference between the 2D and 3D models is in the implicit viscous stress calculation. Recall that for the $2 \mathrm{D}$ basic model, the solver never considers the viscous stress to be converged, so the maximum number of allowable iterations (ITVSMX) is always used. In 3D, the solver occasionally used the maximum number of viscous stress iterations, but it often considered the viscous stress to be converged before ITVSMX was reached, a behavior, which was never observed for the $2 \mathrm{D}$ basic model. The reason for this improved convergence behavior is not known, but perhaps it is due to the additional physical degree of freedom that the jet is allowed while spiraling in the 3D model. Because of time limitations, no FIDAP simulations were performed with the 3D model. 


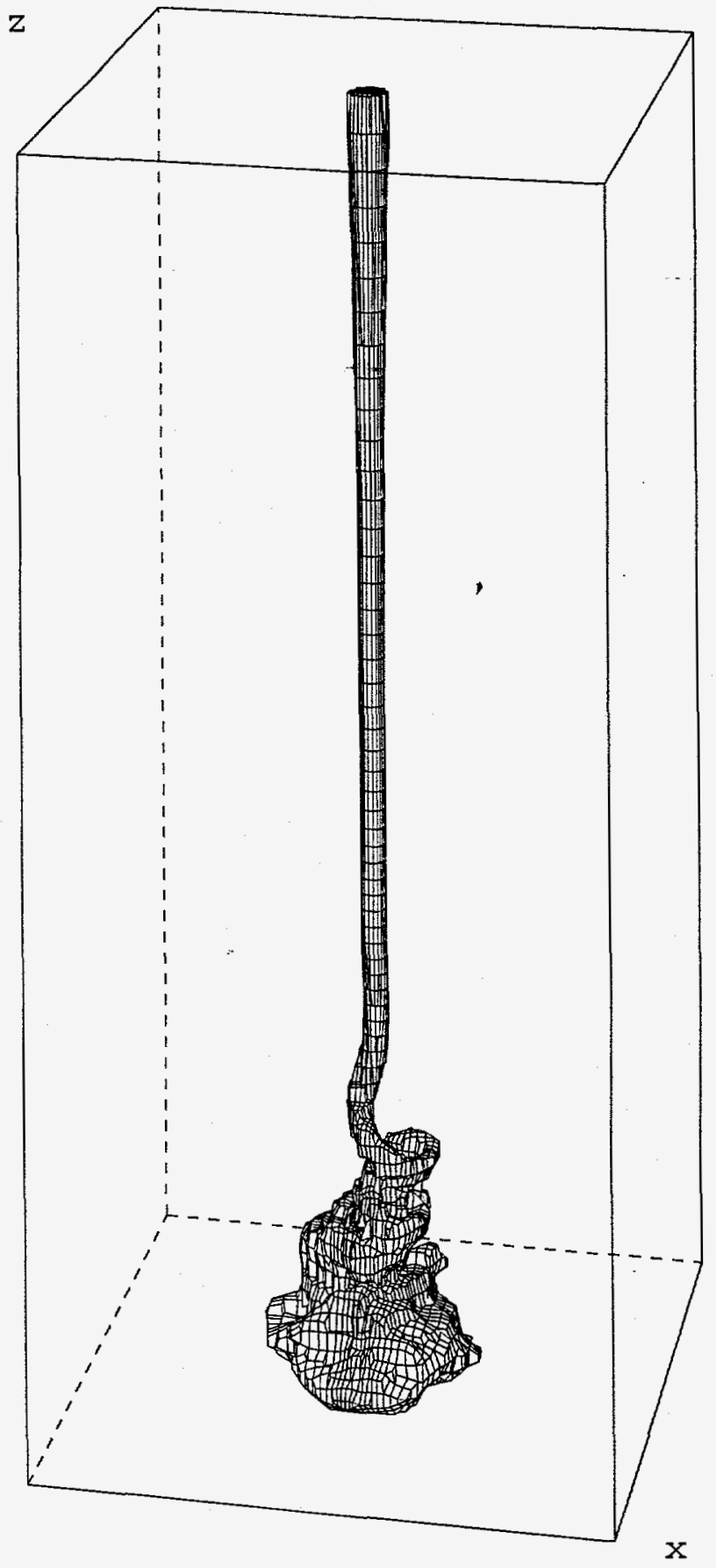

Figure 5.1: $3 \mathrm{D}$ Spiraling Jet 


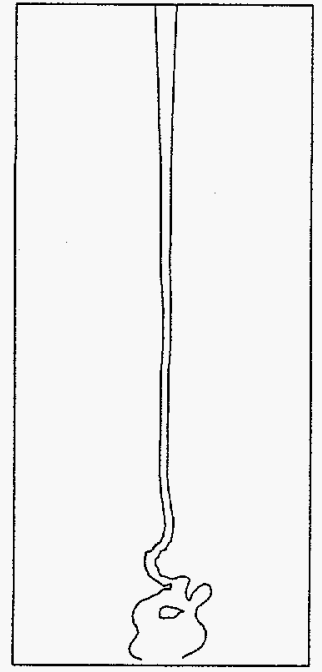

(a)

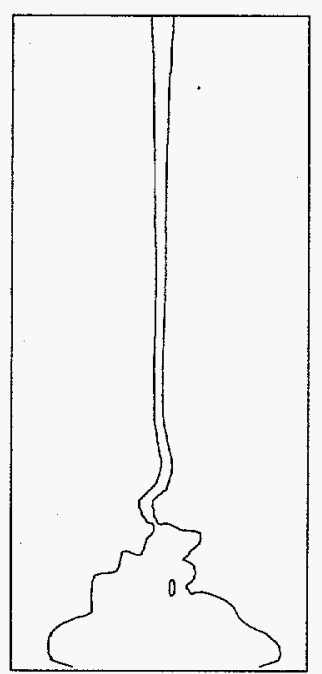

(c)

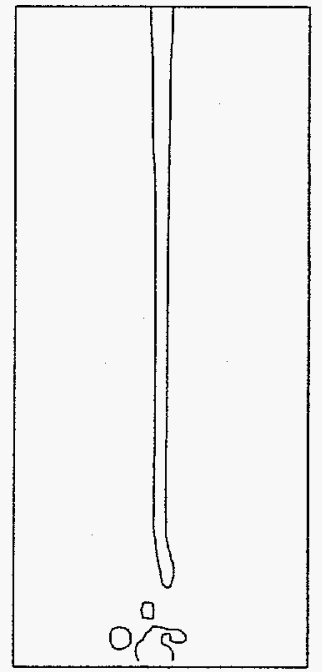

'(b)

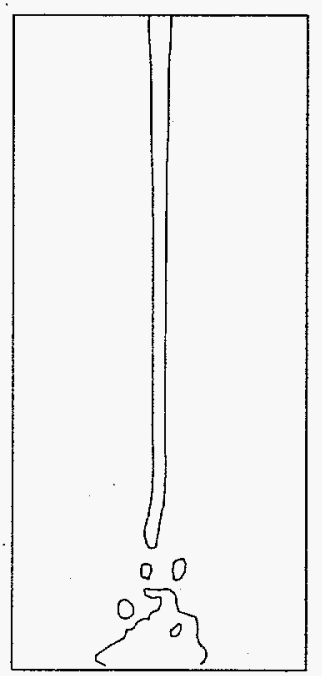

(d)

Figure 5.2: Comparison of $2 \mathrm{D}$ and $3 \mathrm{D}$ Results. (a) $2 \mathrm{D}, \mathrm{t}=0.7 \mathrm{sec}$. (b) $3 \mathrm{D}, \mathrm{t}=0.7 \mathrm{sec}$. (c) $2 \mathrm{D}, \mathrm{t}=$ $1.6 \mathrm{sec}$. (d) $3 \mathrm{D}, \mathrm{t}=1.6 \mathrm{sec}$ 


\section{LARGE JET LENGTH MODEL}

\subsection{DESCRIPTION OF COMPUTATIONAL MODEL}

The focus of this chapter is the "large jet length model." This model is similar to the basic model, which was discussed in Chapter 4, but instead of simulating a small jet length $(34 \mathrm{~cm})$, it simulates a large jet length $(1.7 \mathrm{~m})$. However, the threedimensionality of the previous chapter's model will not be included in this model. Thus the large jet length model has the following characteristics:

- two-dimensional,

- large jet length,

- single fluid, and

- isothermal.

\subsection{EXPERIMENTAL RESULTS}

Several analogous fluid experiments were performed with the experimental apparatus in the "large jet length" configuration pictured in Figure 3.1(b), with a distance of $1.7 \mathrm{~m}$ from the nozzle to the bottom of the receiving container. In general, the qualitative behavior of the flow in the large jet length configuration was similar to the flow in the small jet length configuration. The jet began to neck down immediately upon flowing out of the nozzle, then buckled when it hit the container and began to spiral in a circular motion. A conical pile developed which occasionally became unstable and slumped to the side, and air voids became entrapped in the fluid pool.

In the large jet length configuration, the spiraling frequency for a particular fluid was observed to depend upon only the mass flow rate of the fluid. This result differs from the small jet length configuration, in which the frequency depended upon both the mass flow rate and the pool height. The pool height does not affect the spiraling frequency in the large jet length configuration because in this configuration the jet falls far enough so that its diameter is practically constant - the necking of the jet is not significant near the landing region. In this case, the relatively minor change in jet length caused by the filling of the container has no effect on the jet diameter, and thus it does not affect the spiraling frequency.

In all of the large jet length experiments performed, the frequency was observed to be significantly higher than for the small jet length configuration. Over the course of about 30 experiments in which the frequency was measured, using both 12,500 cSt and 60,000 cSt silicone oil, the mass flow rate was varied from $5 \mathrm{~g} / \mathrm{s}$ to $35 \mathrm{~g} / \mathrm{s}$, and the frequency varied from $150 \mathrm{~Hz}$ to $600 \mathrm{~Hz}$. The frequencies for the large jet length were higher than for the small jet length because the diameter of the jet in the landing region was smaller for the large jet length: since the jet length was increased, there was a greater distance over which the necking occurred, which caused the jet diameter to be smaller.

\subsection{FLOW-3D}

\subsubsection{Boundary Conditions}

With the small jet length

configuration, the entire jet length (from nozzle to container bottom) was modeled. For the large jet length configuration, this approach is impractical: so many grid points would be required in the vertical direction that the time required to calculate an accurate solution would be too great. Therefore, instead of modeling the entire jet, only the bottom portion of the jet (in the landing region) was modeled.

The rectangular domain was chosen to represent the volume within the receiving container, so the bottom, left, and right boundaries of the domain, representing the solid walls of the container, were subject to the no-slip condition. As with the small jet length configuration, a uniform vertical velocity was applied along the top boundary, and solid baffles were used to block the entire boundary except for a small region in the 
center, with a width equal to the diameter of the jet in the landing region, through which the jet was allowed to flow.

To specify these top boundary conditions, it was necessary to determine experimentally the jet diameter and velocity in the landing region (near the bottom of the jet length, as it entered the region which was included in the computational domain). The velocity of the jet at this point was determined by injecting drops of food coloring into the analogous fluid, and using a high-speed camera to track these food coloring drops as they were carried along with the jet through the landing region. Several experiments were performed over a range of mass flow rates from $5 \mathrm{~g} / \mathrm{s}$ to $35 \mathrm{~g} / \mathrm{s}$, and it was found that the jet velocity in the landing region had a constant value of $4.8 \mathrm{~m} / \mathrm{s}$ for all mass flow rates considered. This value was used as the inlet boundary condition for all simulations performed with the large jet length model. This velocity was much larger than the inlet velocities used for the small jet length model, which were in the range of $0.10-0.35 \mathrm{~m} / \mathrm{s}$. After the jet velocity had been measured, the diameter was calculated based on the velocity and the mass flow rate. For the mass flow rates considered in the experiments, the jet diameter was found to be in the range of 0.12 $-0.31 \mathrm{~cm}$, which was much smaller than the inlet diameter used in the small jet length model $(1.18 \mathrm{~cm})$.

\subsubsection{Results}

Several FLOW-3D simulations were performed with the "large jet length" model with $60,000 \mathrm{cSt}$ silicone oil and corn syrup. Some typical results are shown in Figure 6.1. Figure 6.1(a) shows the jet of silicone oil just before it impacts the bottom of the container. The jet does not neck down at all, a result which agrees with experimental observations of the jet behavior in the landing region: in the large jet length experiments, the jet has already fallen about $1.4 \mathrm{~m}$ before entering the portion of the flow which is included in the computational domain, and the majority of the necking occurs near the nozzle (as seen in Figure 4.2(a)). By the time the jet enters the computational domain, it has already necked down to an essentially stable diameter. On the other hand, the jet in the small jet length model necks significantly, since the top boundary of the computational domain in this model represents the outlet of the nozzle, where the majority of the necking occurs. Figure 6.1(b) shows the jet buckling just after impacting the bottom of the container, and Figure 6.1(c) shows the jet after several periods of oscillation have occurred and a pile of fluid has built up. Both of these behaviors were observed in all of the large jet length experiments, and a typical experimental result is shown in Figure 1.1.

To provide data for a quantitative evaluation of the computational model, a large jet length experiment was performed with $60,000 \mathrm{cSt}$ silicone oil. For a mass flow rate of $20.8 \mathrm{~g} / \mathrm{s}$, the experimentally measured spiraling frequency was $189.6 \mathrm{~Hz}$. A large jet length simulation was performed with FLOW-3D, with boundary conditions chosen to match the experiment, and the model predicted a frequency of oscillation of only $71.4 \mathrm{~Hz}$. The grid resolution was increased in both $x$ and $z$ directions in an attempt to approach a robust result, and the predicted frequency jumped to $142.9 \mathrm{~Hz}$. (The results shown in Figure 6.1 are from this model.) However, when the grid resolution was increased further, the model predicted that the jet did not buckle or oscillate at all - it remained symmetric about the center-line of the container, as shown in Figure 6.2(a). The resolution was increased still further, and again the jet did not buckle. This result runs counter to intuition, since one would expect an increase in grid resolution to lead to a more accurate result. This puzzling phenomenon, where an increase in grid resolution caused the jet to stop buckling, was never observed for the small jet length configuration. The cause of this phenomenon is not known. Because of this strange behavior, it was not possible to determine the grid resolution 
required for robustness of the solution, as was done in Section 4.3.2 for the small jet length configuration.

Similar behavior was observed when the value of ITVSMX was varied to determine robustness. While holding the grid resolution and the value of EPSADJ constant, the value of ITVSMX was varied between 100 and 2000. When the value of ITVSMX was set to 100 , the jet buckled and oscillated, with a frequency of $66.7 \mathrm{~Hz}$. With ITVSMX $=500$, the predicted frequency was $142.9 \mathrm{~Hz}$. However, when ITVSMX was increased to 2000 , the jet failed to buckle. This was a surprising result, since, as with the grid resolution, an increase in the value of ITVSMX is expected to lead to a more accurate solution.

The value of EPSADJ was also varied in order to determine the value required for robustness of the solution. When EPSADJ was varied between 0.001 and 1000 , it was found that the solution was identical for all values of EPSADJ $\leq 1$, and when EPSADJ was larger than 1 , the pressure in the jet and pool behaved in ways that were not physically realistic. This result makes sense, considering that EPSADJ = 1 is defined to be the largest value that will guarantee a stable, converged solution for the pressure at each time step.

The fact that changing both the grid resolution and ITVSMX in a way that would be expected to give a more accurate solution caused the jet to stop buckling suggests that the unbuckled solution may be the "correct" solution according to FLOW-3D. However, it is certainly not the result that was obtained experimentally, since the jet always buckled in every experiment, for both the small jet length and large jet length configurations.

All of the problems with FLOW-3D's solution of the small jet length model which were discussed in Section 4.3.4 also apply to the solution of the large jet length model. In addition to these problems, and the problem described in the previous paragraphs in which the jet fails to buckle, there is one other problem observed with FLOW-3D's solution of the large jet length model: in some simulations, soon after the jet impacts the bottom of the container, the pressure in the cells immediately below the jet inlet "blows up." In other words, the pressure at the corners of the inlet suddenly increases by two or three orders of magnitude, and the pressure in the cells immediately below the corners decreases by the same amount. This causes the jet to begin to buckle at this point near the inlet, as shown in Figure 6.2(b). (The buckling location is indicated in the figure with an arrow.) Although the buckling amplitude is very small near the top of the domain, it increases as the jet flows further into 'the domain, and eventually causes the jet to break by the time the buckle reaches the bottom of the container. This type of behavior was never observed experimentally. It was found that this problem can be eliminated by increasing the height of the domain until the jet no longer buckles at the inlet. Like the jet's failure to buckle, this pressure problem was never observed for the small jet length model.

\subsection{FIDAP}

Several FIDAP simulations were performed with corn syrup in the "large jet length" configuration. Figures 6.3(a) and (b) show examples of the most physically realistic results obtained from these FIDAP simulations, and Figures 6.3(c) and (d) show results obtained from FLOW-3D under the same conditions as the FIDAP simulation (same grid resolution, boundary conditions, etc.).

In Figure 6.3(a) the FIDAP jet is shown just before impacting the bottom of the container. At this point in the simulation, the FIDAP solution agrees with the FLOW-3D solution (Figure 6.3(c)) and also with experimental results, which showed for the large jet length configuration that the jet did not neck in the landing region.

Figure 6.3(b) shows the FIDAP solution after the jet has impacted the bottom of the container and buckled. As with 
- previous FIDAP models, the jet buckles far from the bottom of the container, a behavior, which was not observed experimentally. It was hoped that the buckling in FIDAP's large jet length model would be more physically realistic than the small jet length model, since the large jet is thinner and would be expected to buckle more readily. However, both the large and small jet length models predict the same unrealistic buckling behavior. In contrast, the FLOW-3D result shown in Figure 6.3(d), which used the same computational grid as the FIDAP solution, gives a much more realistic prediction of the buckling location, as was the case for the small jet length models. 


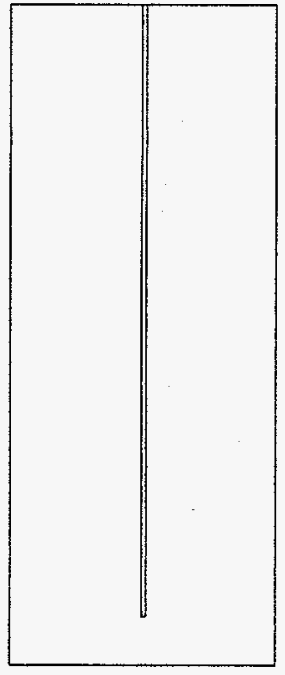

(a)

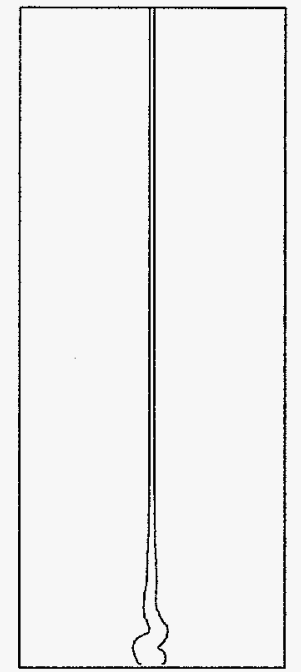

'(b)

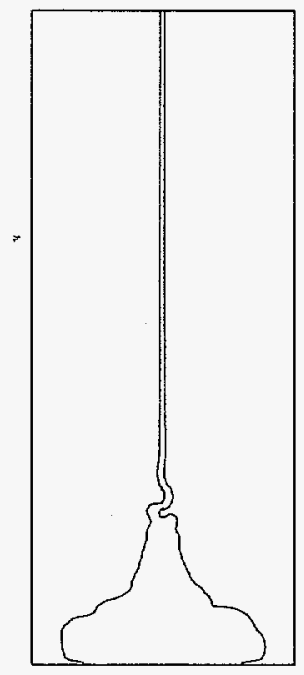

(c)

Figure 6.1: Typical FLOW-3D Results for $60,000 \mathrm{cSt}$ Silicone Oil with Large Jet Length. (a) Just before impact. (b) Initial buckling. (c) Oscillating. 


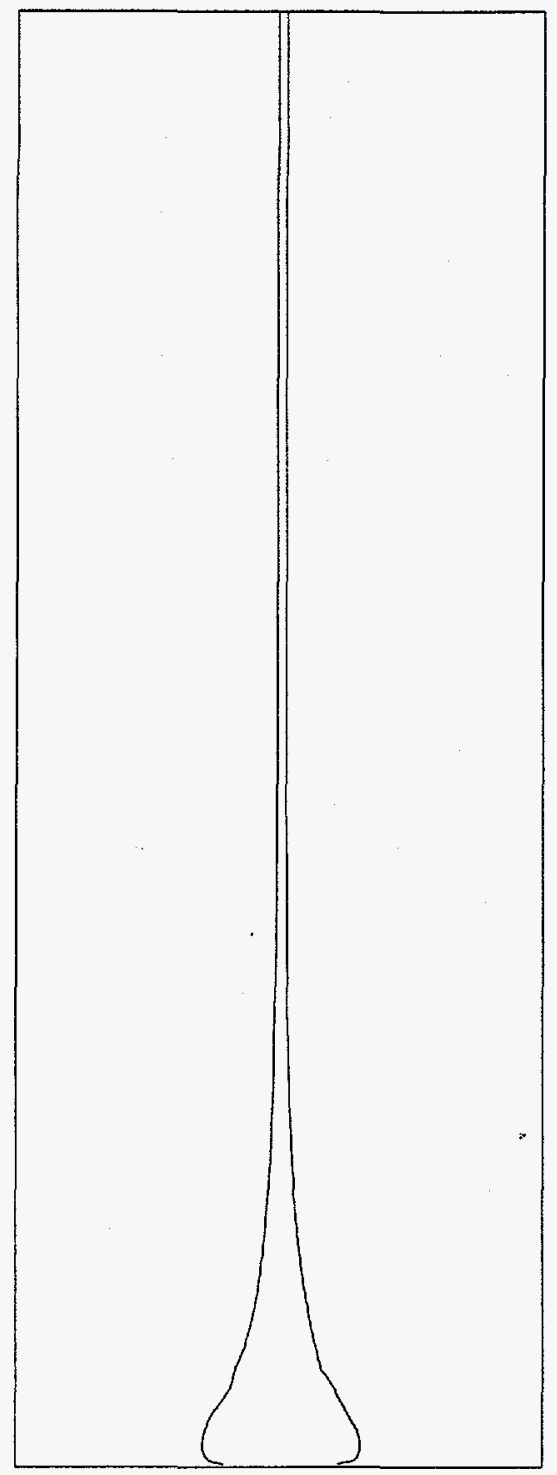

(a)

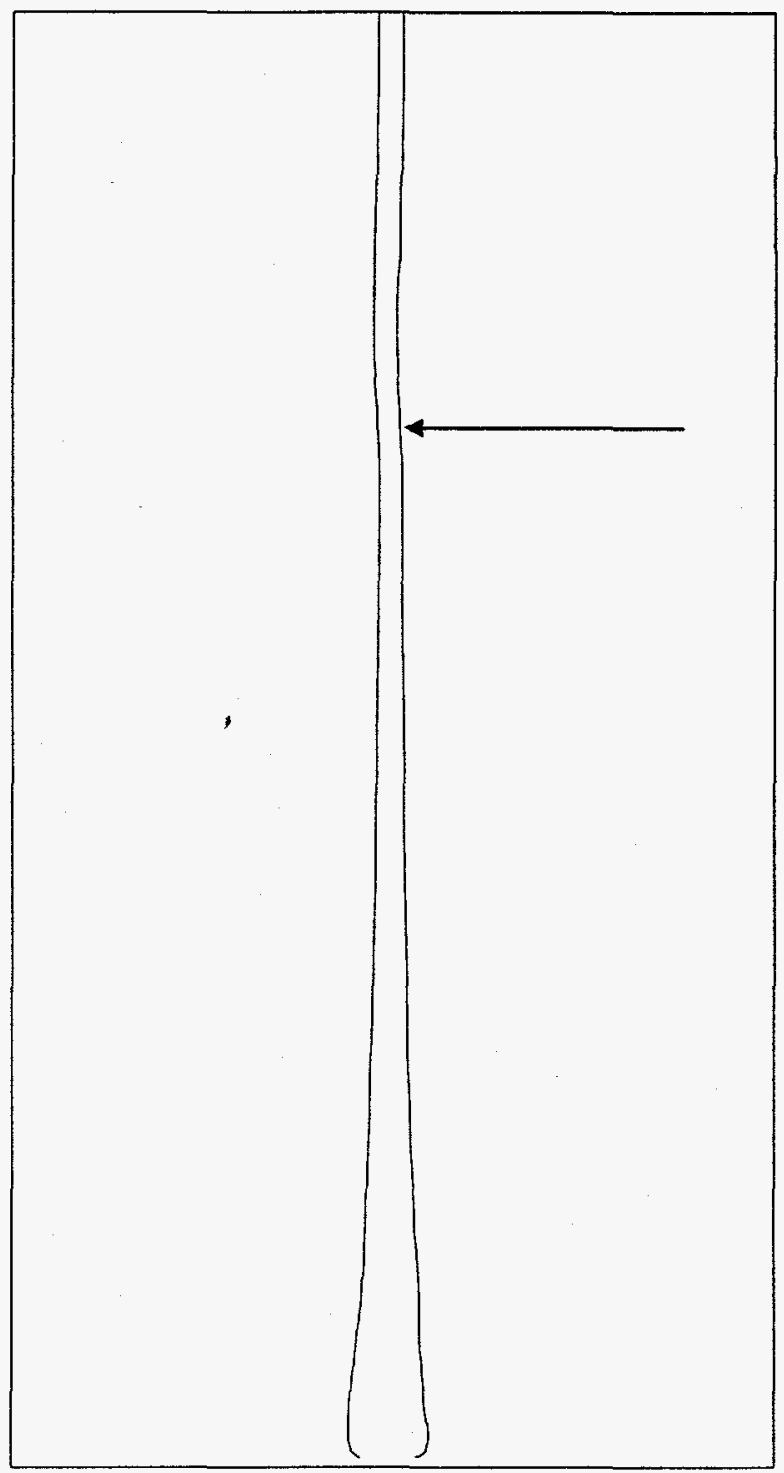

(b)

Figure 6.2: Unrealistic FLOW-3D Results with Large Jet Length Model. (a) No buckling. (b) Buckling near inlet. 


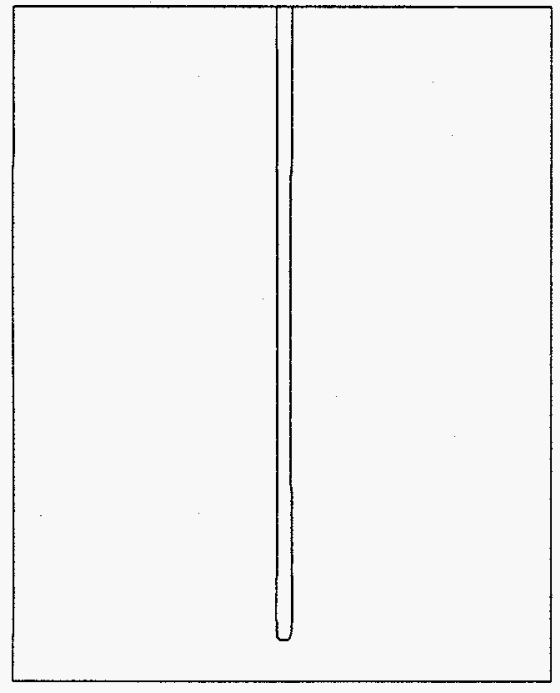

(a)

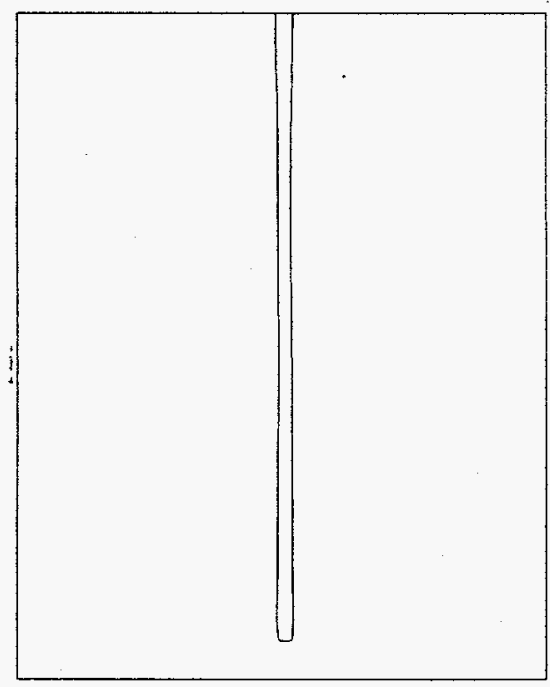

(c)

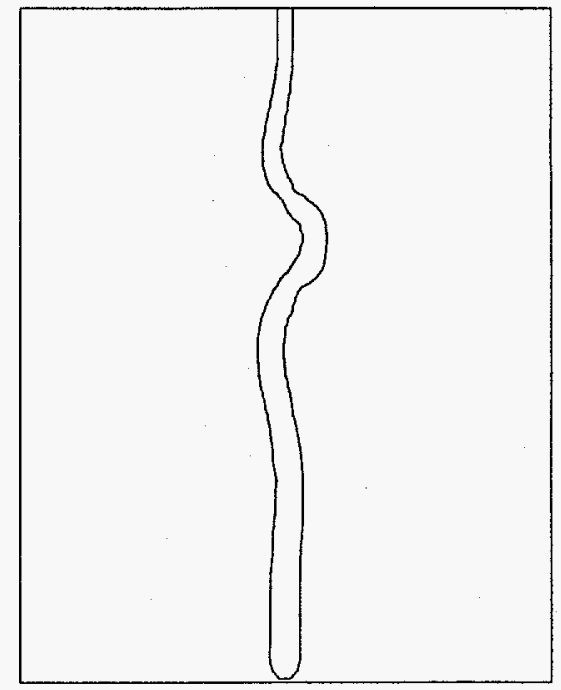

(b)

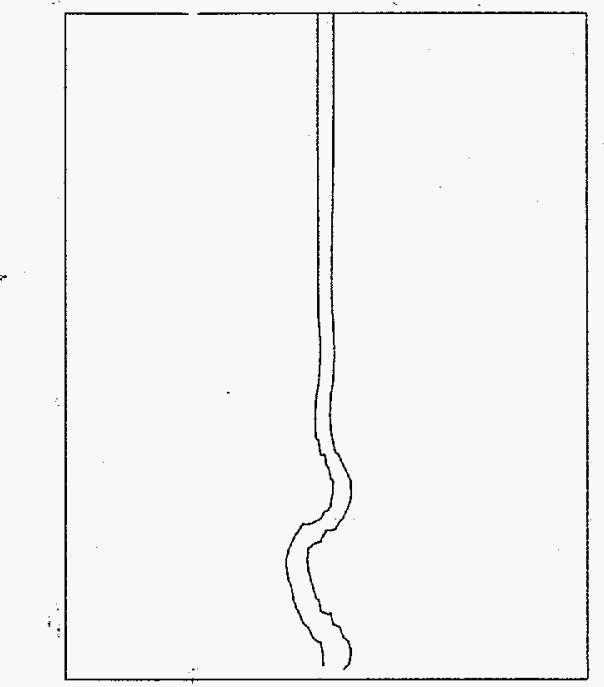

(d)

Figure 6.3: Comparison of FIDAP and FLOW-3D Results for Large Jet Length Model.

(a) FIDAP, just before impact. (b) FIDAP, initial buckling. (c) FLOW-3D, just before impact. (d) FLOW-3D, initial buckling. 



\section{TWO FLUID MODEL}

\subsection{DESCRIPTION OF COMPUTATIONAL MODEL}

The focus of this chapter is the "two fluid model." This model differs from the basic model discussed in Chapter 4 in that instead of modeling the non-jet region of the domain with an imaginary constant-pressure void, this region is filled with a real fluid (air), and the governing equations are solved in the jet and in the air to predict fluid motion throughout the entire domain. The two fluid model has the following characteristics:

- two-dimensional,

- small jet length,

- two fluids, and

- isothermal.

\subsection{FLOW-3D}

\subsubsection{Boundary Conditions}

In all the FLOW-3D models discussed in the previous chapters, there was no way for any fluid to exit the domain: the top boundary was blocked off except for the region through which the jet entered the domain, and the other boundaries represented the solid walls of the container. These conditions were acceptable because the constant-pressure void region, which initially filled the domain simply disappeared when it was replaced by the incoming jet fluid - it did not need to flow out of the domain. However, for the two fluid model, the air which initially fills the domain needs some way to escape as it is displaced by the analogous fluid which is flowing into the domain. In the analogous fluid experiments, the top of the receiving container was open, and air could flow out through that opening. Unfortunately, FLOW$3 \mathrm{D}$ requires that the entire top boundary must have the same boundary condition applied, so it is not possible to allow the air to flow out while the jet is flowing in.
To circumvent this difficulty, it was decided to allow the air to flow out the sides of the domain. The no-slip condition which had been applied along these boundaries was replaced by the continuous condition, which allows fluid to flow freely out of the domain. If it is desired to run the solution for a long time to simulate the filling of the container, solid baffles can be added to the lower part of the side boundaries to simulate the container walls, while leaving the upper part of the side boundaries open to allow the displaced air to escape the domain. Since the motion of the air is not expected to have a significant effect on the jet flow, it is believed that this compromise allows for a physically realistic model.

\subsubsection{Results}

Given the success with which FLOW3D simulated the single fluid, small jet length flow, it was anticipated that the addition of the second fluid to this model would not introduce any significant problems. However, this was not the case. Figure 7.1(a) shows the pressure contours in the jet and the air as the jet falls toward the bottom of the container. It is seen that the addition of the air to the model creates an unstable "checkerboard" pressure distribution within the jet, with regions of high and low pressure alternating along the length of the jet. This is not a physically realistic pressure distribution, and this type of pressure distribution was never observed in the single fluid FLOW-3D models. Figure 7.1(b) shows the effect of this checkerboard pressure distribution: the high and low pressure regions cause the jet to buckle while still in midair, before impacting the bottom of the container. As the solution proceeds in time, these oscillations increase in amplitude until the jet breaks up or the pressure fails to converge. This mid-air buckling and breaking of the jet was never observed in the analogous fluid experiments or the single fluid FLOW$3 \mathrm{D}$ models.

Selected computational parameters were changed in an attempt to stabilize the 
solution. The density of the air in the model was set to several different values, ranging from five orders of magnitude larger than its real physical value to five orders of magnitude smaller than its real physical value. The viscosity of the air in the model was also increased above its real physical value, and decreased below its real physical value. An initial slug of analogous fluid, with a vertical velocity equal to the inlet velocity, was used as the initial condition. (This is the configuration shown in Figure 7.1(a).) The effects of surface tension were removed from the model. The computational parameter OMEGA was set equal to one, to increase the amount of relaxation used in the iterative calculation of the pressure. The value of EPSADJ was decreased to values as low as $10^{-6}$. These changes affected the solution in a variety of ways, but none of them eliminated the erroneous pressure distribution. The cause of the strange pressure solution is not known, and due to time constraints, this issue could not be fully resolved.

\subsection{FIDAP}

Recall that in Section 4.4.2, it was shown that FIDAP did not perform well when simulating the "basic model" of Chapter 4, which included only a single fluid. It was hoped that the cause of this unrealistic result was the exclusion of the effects of air in the model. Unfortunately, the solution was unchanged when the second fluid was added to the model. The FIDAP results calculated with the two fluid model of this chapter were identical to the results calculated with the basic model, shown in Figure 4.9. These results do not agree with any of the analogous fluid experiments.

Two problems arose when using the two fluid model with FIDAP, which did not occur when using the single fluid models. The first problem was that the magnitude of the velocity in the air near the corners of the jet inlet gradually increased throughout the course of the simulations, and eventually this velocity grew so large that the computations halted. It was found that increasing the viscosity of the air in the computations, so that it was ten times larger than the actual viscosity of physical air, eliminated this problem. It is believed that this change does not significantly affect the solution, since the viscosities of the analogous fluids considered in this work are six to seven orders of magnitude larger than the viscosity of air, so a change of one order of magnitude in the air viscosity is minor.

The other problem that was observed in FIDAP's solution of the two fluid model was an erroneous implementation of the effects of surface tension. Figure 7.2(a) is a close-up of the tip of the falling jet, showing velocity vectors in $60,000 \mathrm{cSt}$ silicone oil and air, as well as three contours of $F: 0.05,0.50$, and 0.95 . It is seen that the velocity in the air beneath the jet is moving almost three times as fast as the fluid in the jet, and some of it is flowing back up into the jet, which is clearly not a physically realistic situation. (The $F=$ 0.50 contour is considered to be the free surface of the jet, and the $F=0.05$ contour is included to demonstrate that the large velocities are truly in the air and not at the. very tip of the jet.) Figure 7.2(b) shows a corresponding plot for corn syrup, the surface tension of which is almost four times as large as that of silicone oil. This plot shows that the magnitude of the error increases as the surface tension is increased: the velocity in the air beneath the corn syrup is moving almost five times as fast as the corn syrup, and these unrealistically large velocities are seen for several elements downstream of the jet tip. Figure $7.2(\mathrm{c})$, which shows the velocity vectors and $F$ contours for $60,000 \mathrm{cSt}$ silicone oil in a single fluid model, demonstrates that the surface tension does not lead to erroneous velocities unless the second fluid is included in the simulation, and Figure $7.2(\mathrm{~d})$, which is for $60,000 \mathrm{cSt}$ silicone oil and air with the effects of surface tension disabled in the model, demonstrates that the erroneous velocities are caused by the surface tension 
rather than some other characteristic of the model. The two fluid FIDAP simulations were performed both with and without the effects of surface tension, and the shape of the jet was not affected by the change. 


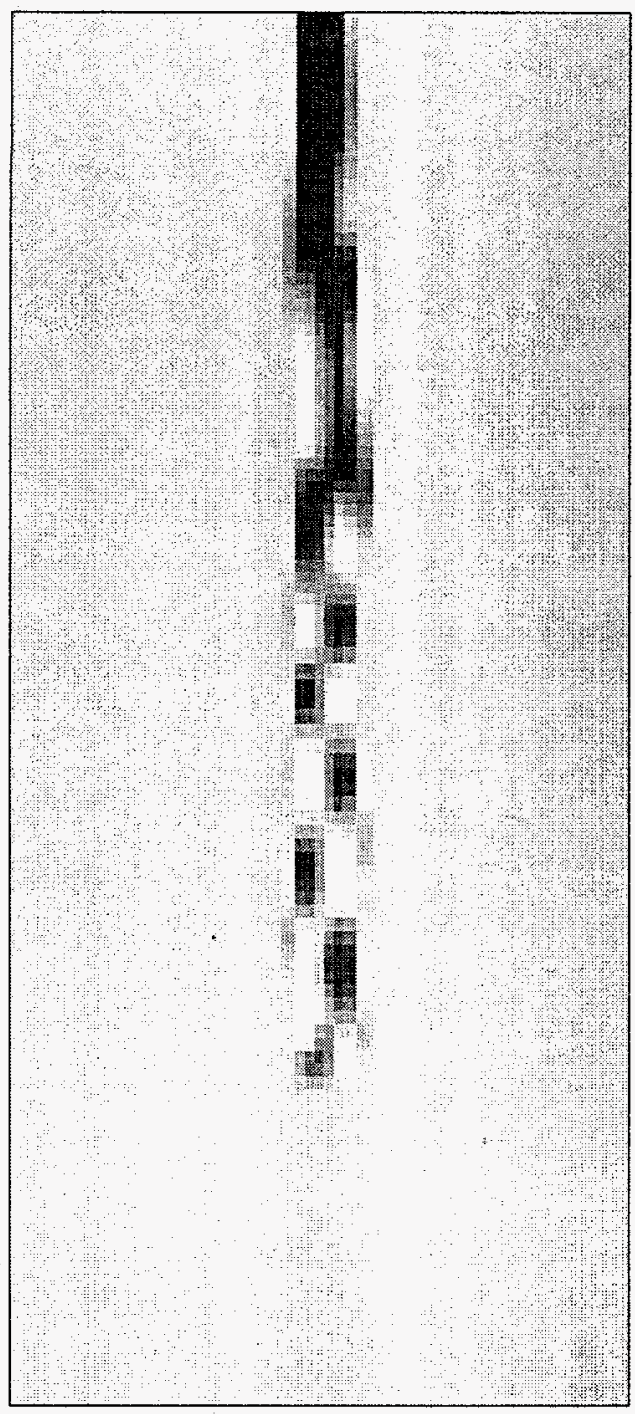

(a)

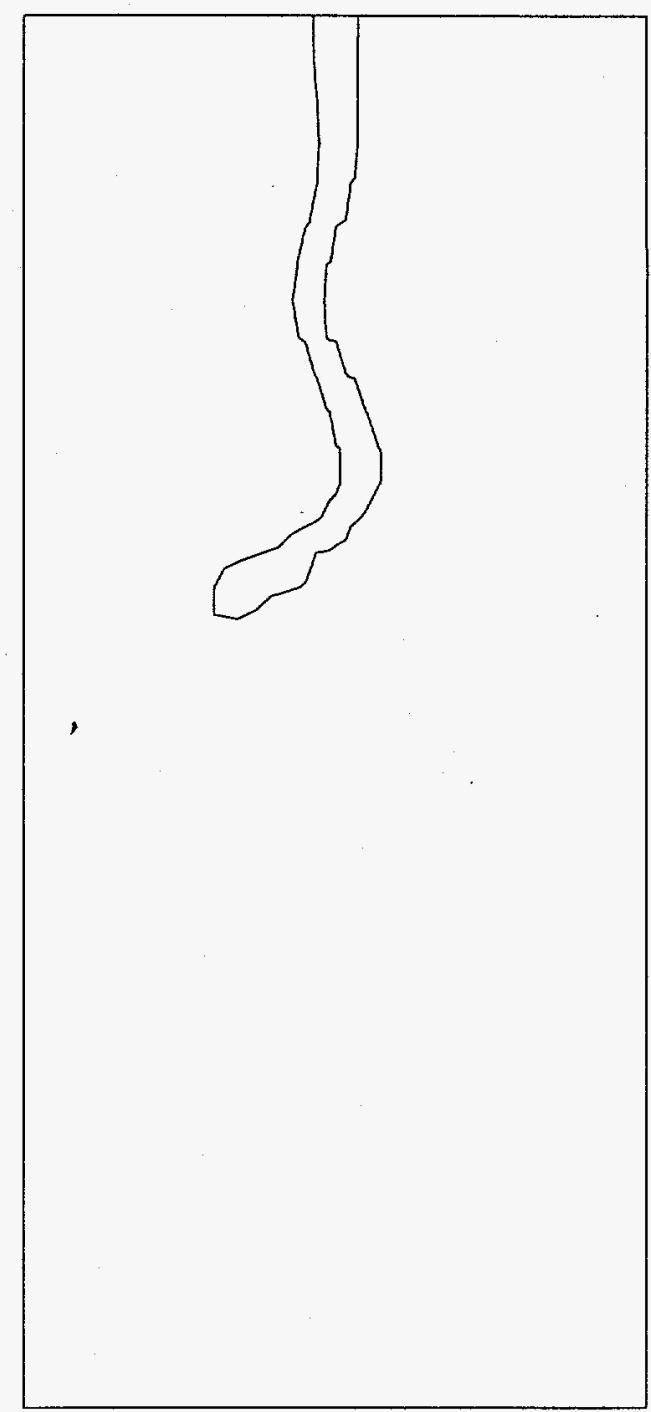

(b)

Figure 7.1: Typical FLOW-3D Results for Two Fluid Model.

(a) Pressure contours (dark regions indicate high pressure, light regions indicate low pressure). (b) Jet buckling in air. 


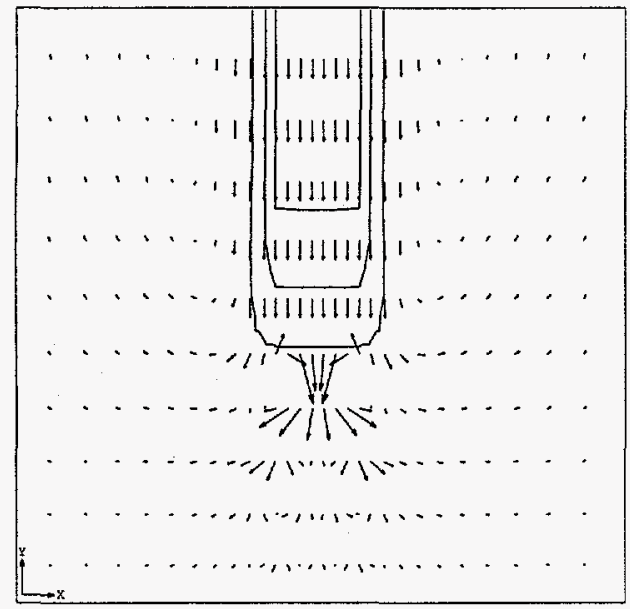

(a)

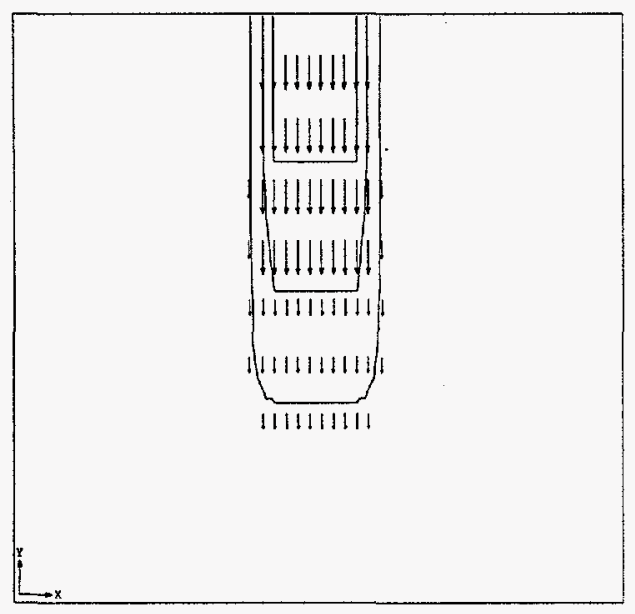

(c)

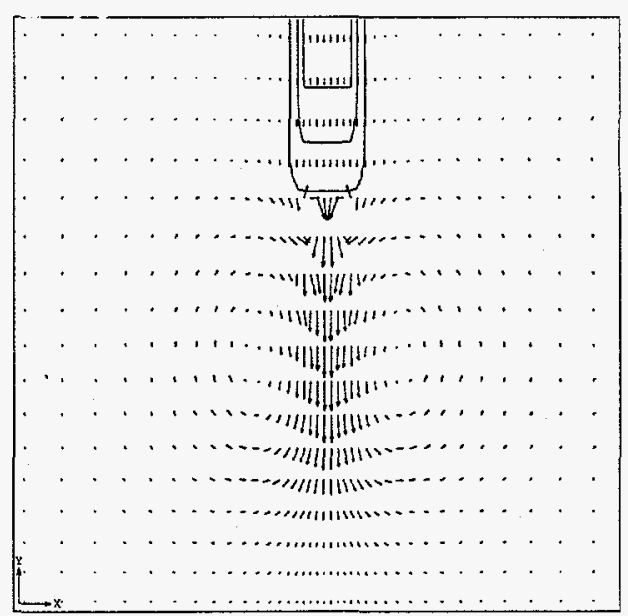

(b)

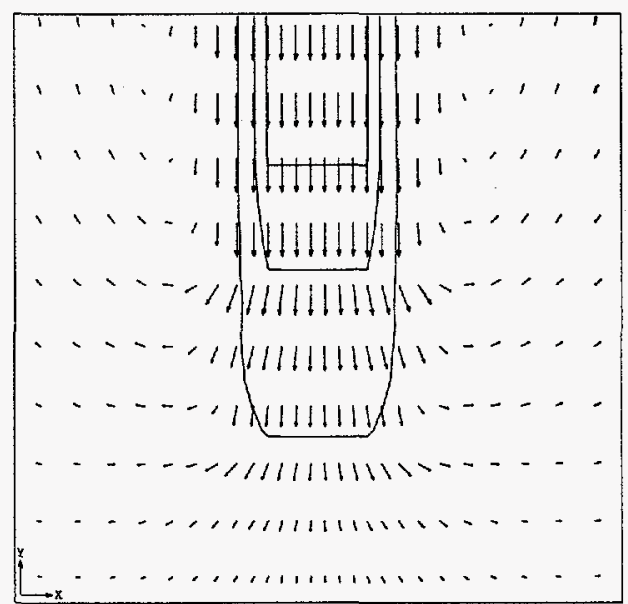

(d)

Figure 7.2: Close-up of Jet Tip during Free-Fall, Demonstrating the Effect of Surface Tension in FIDAP. The three solid lines in each plot represent contours of $F=0.05,0.50$, and 0.95 .

(a) Two fluid model, $60,000 \mathrm{cSt}$ silicone oil. (b) Two fluid model, corn syrup.-.(c) Two fluidmodel, 60,000 cSt silicone oil, no surface tension. (d) Single fluid model, 60,000 cSt silicone oil. 



\section{HEAT TRANSFER MODEL}

\subsection{DESCRIPTION OF \\ COMPUTATIONAL MODEL}

Now that the effects of the second fluid have been added to the model, the next and final stage in the model development is the "heat transfer model." This model includes the effects of heat transfer between the jet, the air, and the receiving container. The heat transfer model has the following characteristics:

- two-dimensional,

- small jet length,

- two fluids, and

- heat transfer.

\subsection{FIDAP}

(The usual pattern in the preceding chapters of this thesis has been to discuss first the FLOW-3D model and results, followed by the FIDAP model and results. However, due to the failure of FLOW-3D to produce any reasonable results with the two fluid model, no solutions were attempted with a FLOW-3D heat transfer model.)

Figures 8.1 and 8.2 show some typical results obtained from the FIDAP heat transfer model: a jet of corn syrup is in mid-fall, prior to impacting the bottom of the container. (Note that, as discussed in Section 7.3, the viscosity of the air in the model is ten times larger than the actual viscosity of real air. This technique was applied to all the models discussed in this chapter.) For this simulation, the air initially within the container was specified to be $18^{\circ} \mathrm{C}$, the initial slug of jet fluid within the container was $23^{\circ} \mathrm{C}$, and the jet fluid flowing into the container was also $23^{\prime \prime} \mathrm{C}$. All fluid properties have constant values, and are not functions of temperature. Figure 8.1 shows the solution at an early time, just after the beginning of the simulation. (The inner solid line is the temperature contour line of $18.5^{\circ} \mathrm{C}$, and the outer line is the $22.5^{\prime \prime} \mathrm{C}$ contour.) The warm jet is heating the cooler air, and the natural convective buoyant force is causing the air to rise on either side of the jet. Even without the aid of buoyancy, the air beneath the jet would tend to flow upward, since it is being forced out of the container by the entering jet; the addition of the buoyant force increases the air's vertical velocity. However, the thermal plumes of air do not rise straight up; they are forced horizontally away from the jet into a "V" shape by the cooler air which is dragged downward in the boundary layer next to the jet. At a later time, shown in Figure 8.2, the thermal plumes have moved in closer to the jet. Since the heated air is accelerated by the buoyant force, it leaves the container faster than the jet enters the container, so other air is forced to flow into the container to conserve the mass of fluid. This air flows in across the top boundary, and some of it is carried back out of the container along with the thermal plumes, forming an eddy near the top boundary on either side of the jet, as shown in Figure 8.2. This recirculation of air forces the thermal plume in toward the jet.

While Figures 8.1 and 8.2 seem to be a physically reasonable result upon initial inspection, there are three problems with them. First, the effect of natural convection in the air appears to be exaggerated. The maximum temperature in the air is $23^{\circ} \mathrm{C}$, which is only $5^{\circ} \mathrm{C}$ above its reference temperature. For this small temperature difference, the buoyant force should be minimal. However, in Figure 8.2, the maximum velocity in the air is more than twice the velocity of the jet, indicating that the natural convection is having a very strong effect upon the air. It may be possible that the vertical velocity of the air is so large because the vertical motion of the air due to its being displaced by the entering jet is acting in combination with the buoyant force, but it seems unlikely that even the combination of these two effects would result in an air velocity which is over twice as large as the jet 
velocity. Therefore, it is believed that the effect of natural convection in the air is overpredicted by FIDAP.

A second difficulty with Figures 8.1 and 8.2 is not observed in the figures because its effect was eliminated prior to solving the problem. In all simulations performed with FIDAP heat transfer models, it was observed that the temperature in the air tended to form a "cellular" distribution, with many small "cells" having locally hot or cold temperatures, as shown in Figure 8.3. It was found that enabling the streamline upwinding option within FIDAP eliminated this problem. Upwinding is a numerical technique which introduces diffusion into a model in order to reduce "node-to-node overshoots and undershoots in the solution variables" (FIDAP 7.6 Update Manual, 1996, p. 2-12). Streamline upwinding was used when generating the solutions shown in Figures 8.1 and 8.2 , so the cellular temperature distribution did not occur.

The third problem with Figures 8.1 and 8.2 is that in some regions of the flow field, the temperature of the fluids is outside of the physically reasonable range of values. Since all the boundary and initial conditions specify temperatures between 18 and $23^{\circ} \mathrm{C}$, it is physically reasonable for all the temperatures in the problem to remain within this range throughout the solution. However, this was not found to be the case. In figures 8.1 and 8.2 , the minimum temperature in the domain is $17.85^{\circ} \mathrm{C}$, and the maximum temperature is $23.002^{\circ} \mathrm{C}$. (The temperature minimum occurs in the air at the left and right "corners" of the lower edge of the thermal plume, where the air turns from its downward flow and begins to rise. The temperature maximum is observed along the left and right vertical edges of the jet, just inside its free surface.) This relatively small temperature error $\left(0.15^{\circ} \mathrm{C}\right)$ may seem like an acceptable amount; however, it was found that when the jet impacted the bottom of the container, the maximum and minimum temperatures began to increase and decrease, respectively. The temperatures continued to increase and decrease without bound, until the solver quit because the time step became too small due to the extremely large temperature values. Thus it was critical to eliminate these unrealistic temperature values before the development of the heat transfer model could proceed.

A very extensive and thorough series of simulations was performed with the heat transfer model using FIDAP. A period of about eight months was devoted to determining and eliminating the cause of the unrealistic temperature values. Several different effects, both physical and numerical, were considered as possible causes of the temperature problem, and the significance of each effect was determined by comparing the temperatures calculated by two simulations, one with the effect included and the other without the effect. When the temperature problem did occur, the temperatures were outside of the physically reasonable range for every time step, so the magnitude of the temperature problem was measured by observing the values of the maximum and minimum temperatures after one time step. However, to monitor the progress of the solutions, each simulation was run for at least 400 time steps, and the maximum and minimum temperature values were also checked at the end of the simulation. The following paragraphs discuss the various effects that were considered, and the significance of each effect on the temperature problem.

1. As mentioned above, the use of streamline upwinding was found to be effective in suppressing the cellular temperature distribution. FIDAP offers two other upwinding techniques, first-order upwinding and hybrid upwinding (FIDAP 7.6 Update Manual, 1996, p. 2-12). It was found that first-order upwinding, which should "virtually eliminate overshoots and undershoots resulting from strong convection" (FIDAP 7.6 Update Manual, 1996, p. 2-28) by introducing a very high level of numerical 
diffusion into the model, increased the effect of the temperature problem, i.e. it caused the maximum and minimum temperatures to be further outside the range of physically reasonable values. Hybrid upwinding, a combination of the streamline and first-order techniques, gave the same temperature error as streamline upwinding. All three upwinding techniques were effective in suppressing the cellular temperature distribution in the air, but none of the three was able to eliminate the temperature problem. It was decided to use streamline upwinding in the remainder of the FIDAP heat transfer models to suppress the cellular temperatures.

2. The grid resolution was varied throughout a wide range of values. Grids with $100,1050,2400,4800$, and 9600 elements were used to simulate the heat transfer model. It was found that the finer grid resolutions increased the effect of the temperature error, rather than reducing the error as was expected. None of the grid resolutions was able to eliminate the temperature problem. In addition, since the boundary layer between the jet and the air is so thin (as seen in Figure 8.2), a very fine grid was used to model a small portion of the domain near the tip of the jet, in order to resolve the flow in the boundary layer. Figure 8.4 shows results obtained with this model: the inner solid line marks the free surface of the jet, and the outer line is the $19^{\circ} \mathrm{C}$ temperature contour. The flow behavior in the boundary layer is clearly resolved. However, this model had a larger temperature error than any of the others discussed in this paragraph, again indicating that a finer grid resolution increases the temperature error.

3 . The size of the time step used in the problem was set to $10^{-3}, 10^{-4}$, and $10^{-5} \mathrm{sec}$. (Recall that in FIDAP, the dimensionless Courant number governs the maximum stable time step size. For the velocities occurring in this flow, the Courant-limited time step size was about $5 \times 10^{-3} \mathrm{sec}$, so all three time step sizes that were considered satisfied the criterion for stability.) The smaller time steps had less error after one time step, because the elapsed time was less. However, when the temperature error was compared at equal times, it was found that the size of the time step had no significant effect on the error.

4. FIDAP offers three different techniques for the iterative solution of the nonlinear problem at each time step. The successive substitution technique exhibits linear convergence and is the most robust; the Newton-Raphson method is less robust, but converges quadratically; and the segregated solution algorithm converges very slowly and is useful for very large problems (FIDAP Theory Manual, 1993, pp. 7-4, 7-5, 7-33). All three solution techniques were used to simulate the flow, and it was found that the choice of solution technique had no significant effect on the temperature error.

5. Three techniques are available to advance the solution in time. The backward Euler technique is first-order accurate and implicit; the trapezoidal technique is secondorder accurate and implicit; and the forward Euler technique is first-order accurate and explicit (FIDAP Theory Manual, 1993, pp. 714, 7-16, 7-19). Each of these three time integration techniques was used in the heat transfer model, and the different techniques had no significant effect on the temperature error.

6. After calculating each iteration, FIDAP uses two criteria to determine whether the solution is converged for a time step: the root-mean-square norm of the difference between the solution at the current iteration and the solution at the previous iteration, divided by the solution at the current iteration, must be less than $\varepsilon_{s}$; and the root-mean-square norm of the residual at the current iteration, divided by the residual at the first iteration, must be less than $\varepsilon_{\mathrm{r}}$ (FIDAP FIPREP Users Manual, 1993, p. 3-79). Both of these criteria must be satisfied simultaneously for the solution to be considered converged. To evaluate the effect of these convergence criteria on the temperature error, the problem 
was solved with $\varepsilon_{\mathrm{s}}=\varepsilon_{\mathrm{r}}=10^{-2}, 10^{-4}, 10^{-7}$, and $10^{-10}$. The size of the convergence criteria was found to have no significant effect on the temperature error.

7. FIDAP includes the use of an acceleration factor to relax the nonlinear iterative solver in hopes of improving convergence. The factor $\alpha$ is used in the equation $\mathbf{U}_{i+1}=\alpha \mathbf{U}_{i}+(1-\alpha) \mathbf{U}^{*}$, where $\mathbf{U}^{*}$ is the solution to $\mathbf{K}\left(\mathbf{U}_{\mathrm{i}}\right) \mathbf{U}^{*}=\mathbf{F}$ (FIDAP FIPREP Users Manual, 1993, p. 3-82). (Note that a value of $\alpha=0$ means that no relaxation will be used.) The problem was solved with $\alpha=0$, $0.5,0.9$, and 0.99 , but the acceleration factor had no significant effect on the temperature error.

8. The problem was first solved with linear basis functions in each element, and then solved with quadratic basis functions. The change in the order of the basis function had no significant effect on the temperature error.

9. In FIDAP, the pressure may be calculated in two different ways. Using the penalty function approach, the continuity equation is discarded from the analysis and the pressure is eliminated from the momentum equation. With the mixed pressure formulation, the pressure is discretized and added to the solution vector of unknowns (FIDAP FIPREP Users Manual, 1993, p. 3-33). Both options were implemented in FIDAP, and it was found that neither implementation eliminated the temperature error.

10. When determining whether an element is completely full of a single fluid, FIDAP uses the parameter $\varepsilon_{f}$ to clean up "misty" elements (elements which are almost full or almost empty) in order to reduce round-off error. In element $i$, if $F_{i}<\varepsilon_{\mathrm{f}}$, then the element is declared to be full of air, and if $F_{i}>1-\varepsilon_{\mathrm{f}}$, the element is declared to be full of jet fluid (FIDAP 7.5 Update Manual, 1995, p. 2-13). Values of $\varepsilon_{\mathrm{f}}=10^{-3}, 10^{-8}$, and $10^{-.12}$ were used, but the changes had no effect on the temperature error.

11. Several different physical conditions were modeled on the top boundary of the domain to see if this would have an effect on the temperature error. In one case, the air was allowed to flow out across the entire top boundary (except for the region where the jet was flowing into the domain) and the temperature flux across the boundary was specified to be zero. The second case was similar, but instead of specifying the temperature flux to be zero, the temperature was specified to be $18^{\circ} \mathrm{C}$. Two other cases modeled the jet falling through the center of a pipe' without touching the pipe walls: a small region of the top boundary on either side of the jet had a specified linear temperature gradient from $23^{\circ} \mathrm{C}$ at the jet free surface to $18^{\circ} \mathrm{C}$ at the pipe wall, and the remainder of the top boundary had either a temperature flux of zero or a temperature of $18^{\circ} \mathrm{C}$ specified. The final four cases had solid horizontal walls along the top boundary on both sides of the jet inlet (with either a temperature flux of zero or a temperature of $18^{\circ} \mathrm{C}$ along the walls), and a small gap on the outer end of each wall to allow the air to exit the domain near the container side walls (with either a temperature flux of zero or a temperature of $18^{\circ} \mathrm{C}$ in these gaps). In total, the various combinations of these options accounted for eight different models. When the results from these models were compared, it was found that the models did of course have a significant affect on the velocity and temperature fields, but none of the sets of boundary conditions was able to eliminate the temperature error.

12. The initial and boundary conditions were changed to provide a temperature difference between the jet and the air of $5^{\circ} \mathrm{C}, 1^{\circ} \mathrm{C}$, and $0.1^{\prime \prime} \mathrm{C}$. It was found that the magnitude of the temperature error scaled with the magnitude of the temperature difference between the two fluids, but none of 
the temperature differences was small enough to eliminate the temperature error altogether.

13. The boundary condition governing the speed of the jet at the inlet was set to several different values, ranging from 5 to $64 \mathrm{~cm} / \mathrm{s}$. The jet inlet speed was found to have no effect on the temperature error.

14. The problem was solved with the Boussinesq term included in the analysis to account for the natural convection buoyant force, and then the effect of natural convection was eliminated from the problem. It was hoped that the model without natural convection would yield a more accurate solution due to the decreased coupling between the temperature and momentum equations. Removing the natural convection prevented the thermal plume in the air from rising, as expected, but it had no significant effect on the temperature error.

15. The units of temperature were changed from degrees Celsius to Kelvins, and the units of heat were changed from Joules to calories. Neither of these changes had any effect on the temperature error.

16. The viscosity of the air was increased by one, two, and three orders of magnitude over its real physical value. (Recall that the viscosity of the air used in all the FIDAP heat transfer models was an order of magnitude larger than the real value of the viscosity of air.) The surface tension between the corn syrup and the air was decreased in magnitude, and then eliminated altogether. Neither of these changes significantly affected the temperature error.

17. Over a series of nine simulations, the thermal conductivities of the corn syrup and air were each set to three different values, as shown in Table 8.1: the real physical value, three orders of magnitude larger than the physical value, and three orders of magnitude smaller than the physical value. It was found that the temperature error could be eliminated by increasing the thermal conductivity of the fluids. When the thermal conductivity of the air was increased by three orders of magnitude, the error in the minimum temperature was zero (i.e., the minimum temperature was exactly $18^{\circ} \mathrm{C}$ ) for every time step. When the thermal conductivity of the corn syrup was increased by three orders of magnitude, the error in the maximum temperature became zero within the first 0.1 $\mathrm{sec}$, and remained zero for the rest of the simulation.

This result suggests that the temperature error may be due to a convective instability. These instabilities occur when the cell Peclet number,

$P e=\frac{\sqrt{u^{2}+v^{2}} \Delta x \rho c_{p}}{k}$,

becomes too large, so increasing the thermal conductivity $k$ would decrease the value of $P e$ and tend to stabilize the solution. However, recall that it was found that increasing the grid resolution caused the temperature error to increase. Since an increase in grid resolution means a decrease in grid spacing $\Delta x$, and thus a decrease in $P e$, it would be expected to have the same effect as increasing the thermal conductivity, if the cause of the temperature error was a convective instability. Since increasing the resolution and increasing the thermal conductivity were found to have the opposite effect on the temperature error, it was concluded that convective instability was not the cause of the temperature error.

Although increasing the thermal conductivities of the fluids was found to eliminate the temperature error, it was decided that this was not an acceptable solution to the problem. When the viscosity of the air was increased by one order of magnitude to prevent an instability in the velocity of the air near the corners of the jet inlet, as discussed in section 7.3 , this was deemed to be acceptable because the viscosities of the analogous fluids were still five to six orders of magnitude larger than the modified air viscosity, so the change in the air viscosity had almost no effect on the solution. However, increasing both thermal conductivity values would significantly 
change the resulting temperature solution. For example, the rate of cooling of the jet would be increased, which would dramatically affect the jet's viscosity and change the entire flow field.

18. FIDAP includes an option to clip the value of any solution variable. This means that the user may specify a maximum and minimum acceptable value for any variable, and after the solution has been calculated for a time step, any nodal point values of that variable that lie outside the clipped range are reset to lie within the range. In other words, if the temperature was clipped at $18^{\circ} \mathrm{C}$ and $23^{\circ} \mathrm{C}$, any temperature values that were calculated to be lower than $18^{\circ} \mathrm{C}$ would be set equal to $18^{\circ} \mathrm{C}$, and temperatures higher than $23^{\circ} \mathrm{C}$ would be set equal to $23^{\circ} \mathrm{C}$. This technique was applied to the model, and it was found to eliminate the temperature error if the temperatures were clipped at $18^{\circ} \mathrm{C}$ and $23^{\circ} \mathrm{C}$. When the problem was simulated for an extended period of time, the temperatures remained within the specified range, and the solution seemed qualitatively reasonable.

-However, if the clipping levels were set to be outside the range of physically reasonable values (e.g., clipping at $17.5^{\circ} \mathrm{C}$ and $23.5^{\circ} \mathrm{C}$ ), the maximum and minimum temperature in the domain gradually increased and decreased, respectively, until they reached the clipping levels, and remained there for the rest of the simulation. Also, if the problem was simulated for a time with the temperatures clipped, and then the clipping was disabled, the maximum and minimum temperatures immediately began to increase and decrease, respectively, outside the range of physically reasonable values. Both of these behaviors suggest that clipping the temperatures merely corrects the symptoms of the temperature error, and does not really fix the problem itself. There is no guarantee that the solution obtained when clipping the temperatures is the correct solution to the problem, or that the temperature will remain stable as the solution proceeds in time.

19. Finally, in an attempt to determine the origin of the erroneous temperature values, the corn syrup in the simulations was replaced by air, so the model simulated a jet of warm air at $23^{\circ} \mathrm{C}$ entering a container full of cooler air at $18^{\circ} \mathrm{C}$. Since there was only one fluid in the problem, the VOF method was not used in this simulation. It was found that the physically unrealistic values of temperature still occurred even though the corn syrup had been eliminated from the problem. The magnitude of the temperature error was not as great as in most of the corn syrup runs, but the maximum and minimum temperatures in the domain were both outside of the acceptable range at every time step. This indicates that the temperature error is not caused by the use of the VOF method; rather, it is due to an inherent limitation in FIDAP's implementation of the energy equation.

In summary, it was found that the unrealistic maximum and minimum temperature values occurring in the FIDAP heat transfer models could be eliminated either by clipping the temperature results to specify the range of temperatures allowed in the solution, or by increasing the thermal conductivities of the two fluids. However; neither of these techniques was judged to be an acceptable means of eliminating the error. Ultimately, no acceptable solution was obtained, and it was concluded that FIDAP is incapable of incorporating heat transfer into the impinging jet model. 
Table 8.1: Effect of Thermal Conductivity on Temperature Error

\begin{tabular}{|c|c|c|c|c|c|}
\hline $\begin{array}{c}\text { Multiplier for } \\
\text { Corn Syrup } \\
\text { Conductivity }\end{array}$ & $\begin{array}{c}\text { Multiplier for } \\
\text { Air } \\
\text { Conductivity }\end{array}$ & $\begin{array}{c}\text { Error in min } T \\
\text { after one step } \\
(\mathbf{K})\end{array}$ & $\begin{array}{c}\text { Error in max } T \\
\text { after one step } \\
(\mathbf{K})\end{array}$ & $\begin{array}{c}\text { Error in min } \boldsymbol{T} \\
\text { after } \mathbf{0 . 1} \text { sec (K) }\end{array}$ & $\begin{array}{c}\text { Error in max } T \\
\text { after 0.1 sec (K) }\end{array}$ \\
\hline $10^{-3}$ & $10^{-3}$ & 0.219 & 0.003 & 0.858 & 0.0001 \\
\hline $10^{-3}$ & 1 & 0.219 & 0.003 & 0.419 & 0.002 \\
\hline $10^{-3}$ & $\mathbf{1 0}^{\mathbf{3}}$ & $\mathbf{0 . 0 0 0}$ & 0.008 & $\mathbf{0 . 0 0 0}$ & 0.129 \\
\hline 1 & $10^{-3}$ & 0.219 & 0.003 & 0.862 & 0.0001 \\
\hline 1 & 1 & 0.219 & 0.003 & 0.416 & 0.002 \\
\hline 1 & $\mathbf{1 0}^{\mathbf{3}}$ & $\mathbf{0 . 0 0 0}$ & 0.008 & $\mathbf{0 . 0 0 0}$ & 0.116 \\
\hline $\mathbf{1 0}^{\mathbf{3}}$ & $10^{-3}$ & 0.219 & 0.004 & 11.664 & $\mathbf{0 . 0 0 0}$ \\
\hline $\mathbf{1 0}^{\mathbf{3}}$ & 1 & 0.219 & 0.004 & 3.179 & $\mathbf{0 . 0 0 0}$ \\
\hline $\mathbf{1 0}^{\mathbf{3}}$ & $\mathbf{1 0}^{\mathbf{3}}$ & $\mathbf{0 . 0 0 0}$ & 0.005 & $\mathbf{0 . 0 0 0}$ & $\mathbf{0 . 0 0 0}$ \\
\hline
\end{tabular}




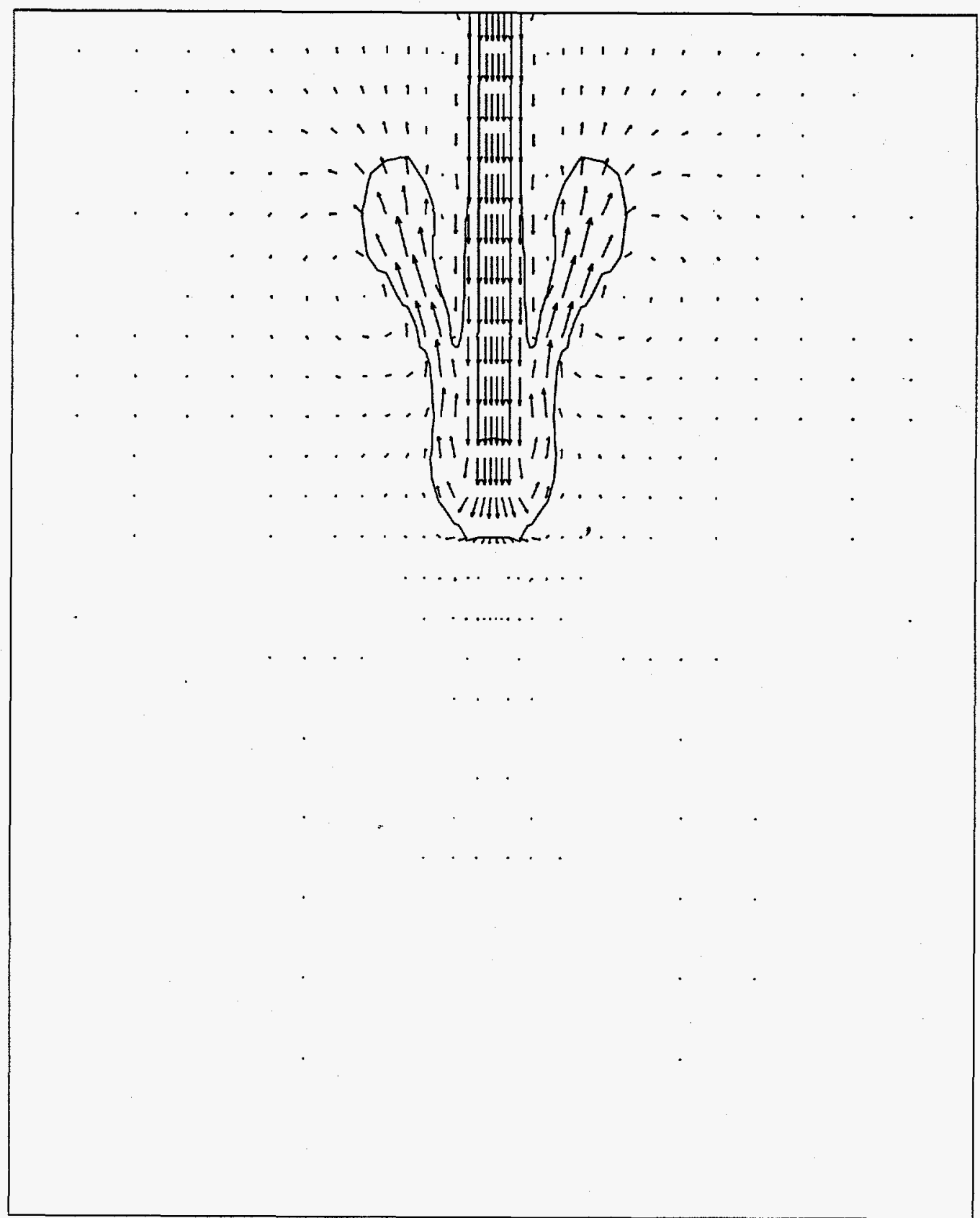

Figure 8.1: Typical FIDAP Heat Transfer Model Results Solid lines are temperature contours of 18.5 and $22.5^{\circ} \mathrm{C} . \mathrm{t}=0.05 \mathrm{sec}$. 


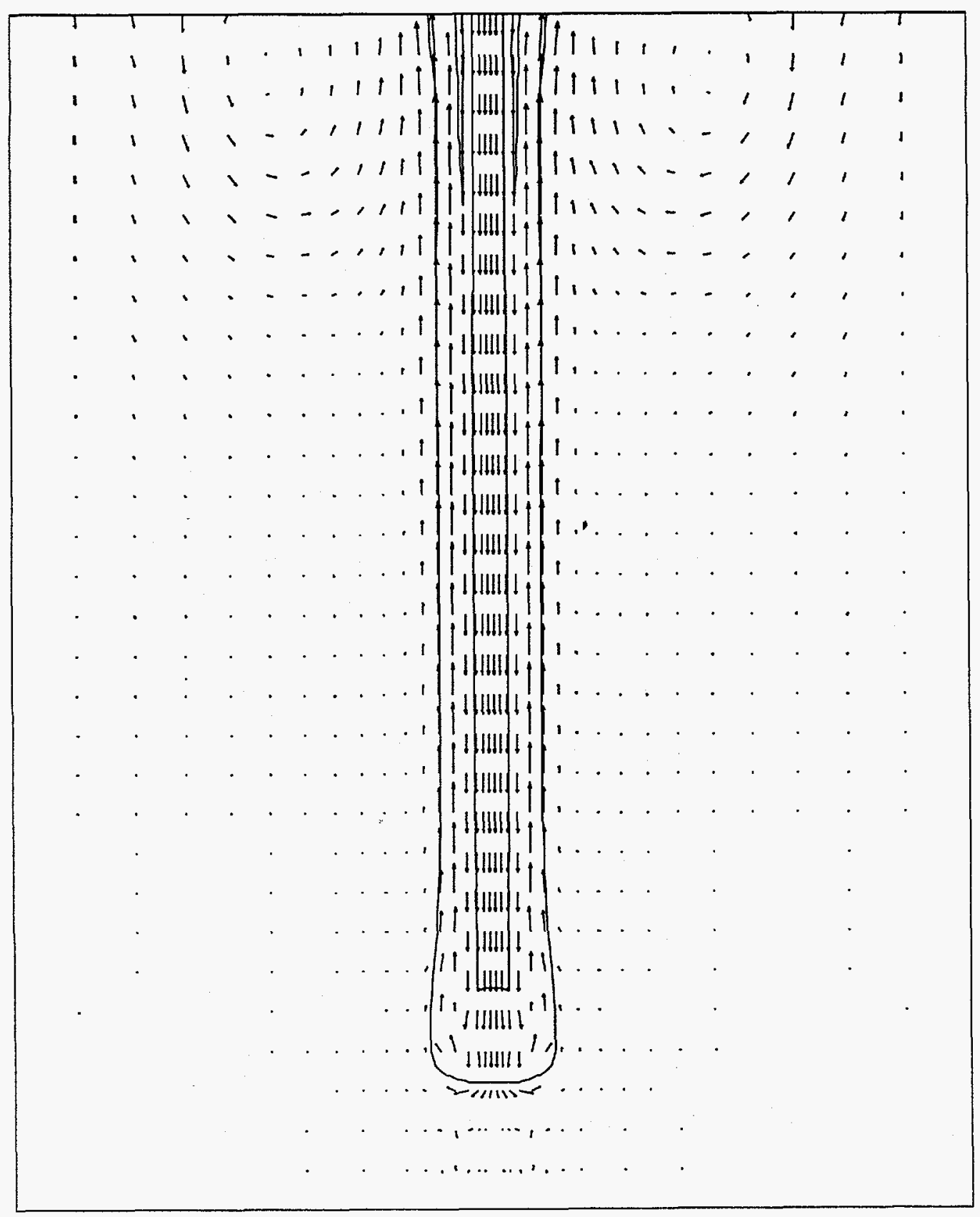

Figure 8.2: Typical FIDAP Heat Transfer Model Results Solid lines are temperature contours of 18.5 and $22.5^{\circ} \mathrm{C} . \mathrm{t}=0.20 \mathrm{sec}$. 


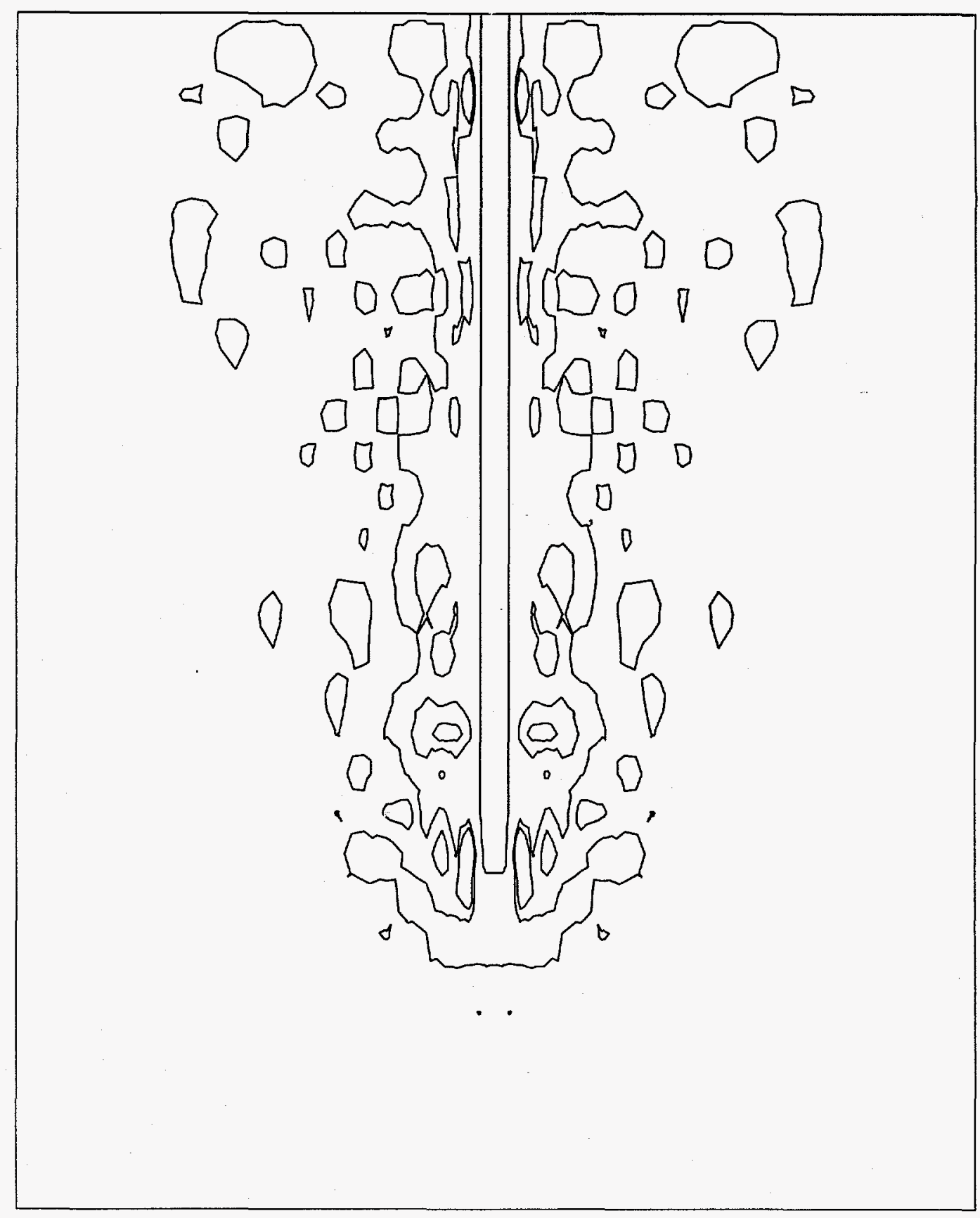

Figure 8.3: Example of "Cellular" Temperature Distribution 


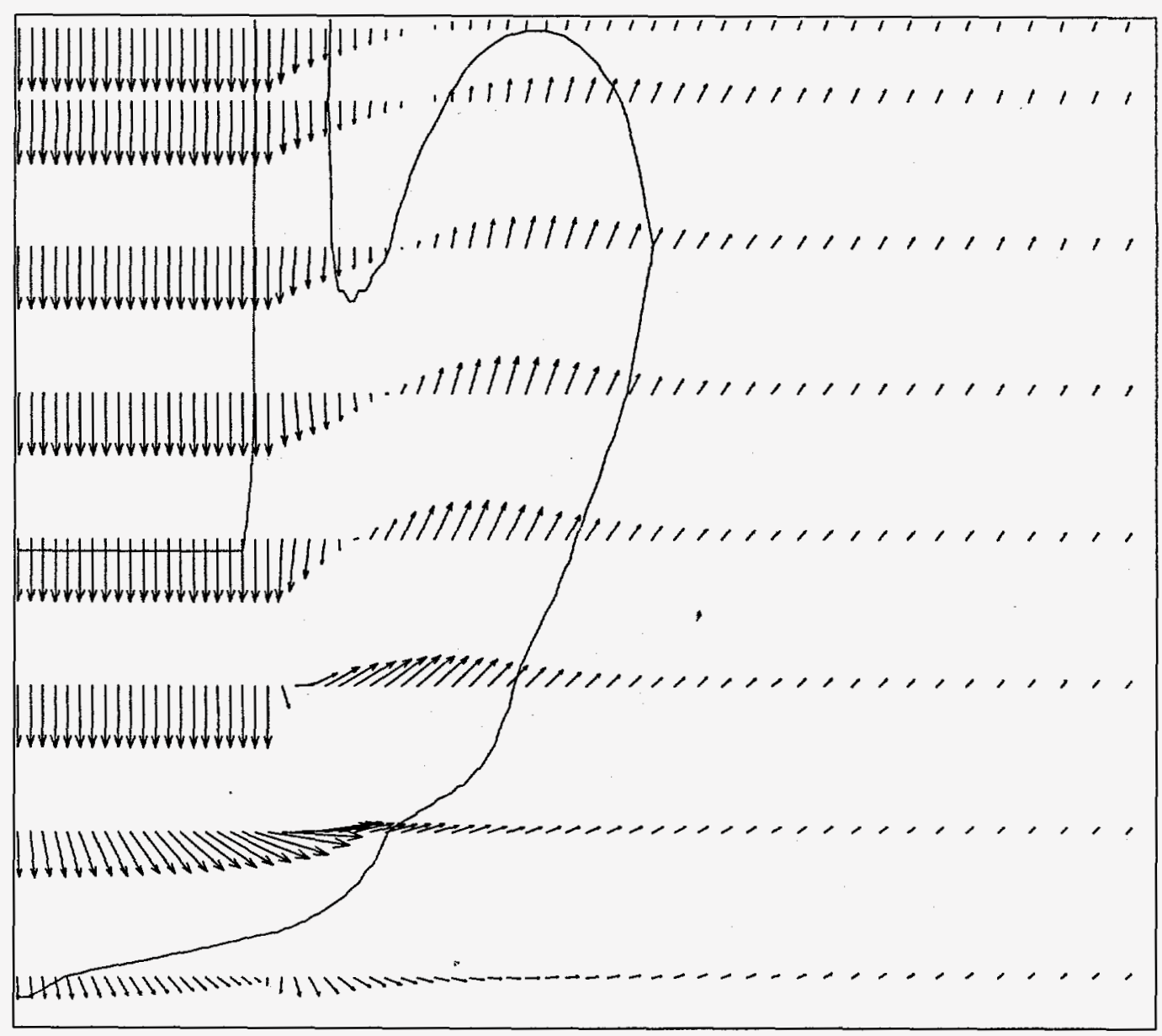

Figure 8.4: Close-up of Flow Near Tip of Jet.

Inner line is free surface; outer line is temperature contour of $19^{\circ} \mathrm{C}$. 



\section{CONCLUSIONS AND RECOMMENDATIONS}

\subsection{CONCLUSIONS}

Several computational models, with varying degrees of physical realism, have been developed to simulate the flow of impinging jets of highly viscous fluids. These models generated mixed results - some of the models agreed extremely well with experimental observations, whereas others gave solutions, which were physically unreasonable.

Of the two CFD codes used in this work, FLOW-3D generally performed better than FIDAP. Most of the FLOW-3D models were able to match qualitatively all of the significant behaviors observed in real impinging jets: necking, buckling, spiraling, slumping, and void entrapment. Both $2 \mathrm{D}$ and 3D models matched all of these significant behaviors. One FLOW-3D model in particular was able to predict quantitatively the spiraling frequency of the jet with only $3 \%$ error. This $2 \mathrm{D}$ model was shown to be robust with respect to the grid resolution, the viscous stress iterations, and the convergence criterion. It was found using FLOW-3D that 2D and 3D models predict the same frequency of oscillation of the jet; however, the results differ in other ways such as effective fill rate and void entrapment mechanism.

However, there are still problems with the performance of the FLOW-3D models. The most significant of these is the failure of the FLOW-3D model when a second fluid, air, was added to the model. The FLOW-3D model did not produce any physically reasonable results when the effects of air were included. Due to time constraints, it was not possible to investigate this problem in depth, and it is believed that a stable, physically reasonable solution will eventually be achieved using FLOW-3D with the two fluid model. The other significant problem with the FLOW-3D results was the jet's failure to buckle when the grid resolution and number of viscous stress iterations were increased for the large jet length. Because of this problem, it was not possible to obtain a robust result for this geometry.

Unlike FLOW-3D, none of the FIDAP models accurately predicted the jet behavior: the jets in all of the FIDAP models did buckle and oscillate, but the point on the jet where the buckling occurred was always far from the bottom of the container, a behavior which was never observed experimentally. FIDAP was able to generate stable results for the two fluid model, which FLOW-3D has thus far been unable to do. The biggest problem with the FIDAP models is the inability to incorporate heat transfer effects into the analysis. Every conceivable technique was applied in an exhaustive effort to correct the physically unreasonable temperature values, but none of the acceptable remedies were effective. Thus, it is concluded that FIDAP is incapable of calculating the heat transfer for the impinging jet problem; since calculation of the heat transfer is a critical stage in the development of the model, FIDAP is not an acceptable tool to use for this work, and future model development should be carried out with FLOW-3D.

\subsection{RECOMMENDATIONS FOR FUTURE WORK}

There are several aspects of this work, which would benefit from further attention. One of these aspects is the failure of FLOW3D's two fluid model. This model could be further investigated with the goal of eliminating the erroneous oscillatory pressure field observed in all the FLOW-3D two fluid models up to this point. Once the two fluid model is working satisfactorily, quantitative comparisons of variables such as the jet's frequency of oscillation could be made between the single fluid and two fluid models. The inclusion of the air in the model may. make it possible to obtain quantitative void fraction predictions with the model, but the extremely fine grid resolution required for this task may make this an unreasonable goal. 
With the effects of air properly implemented in the model, heat transfer may then be incorporated. As a first step, the fluid properties may be assumed to be independent of temperature, and then their temperaturedependent effects may later be incorporated into the model. With the heat transfer working correctly, the final stage of model development is the inclusion of the actual molten glass properties, including the effects of thermal radiation heat transfer.

Two other possible future tasks are an investigation into the FLOW-3D large jet length model that failed to buckle when the grid resolution was increased, and further analysis of the similarities and differences between the 2D and 3D models. 


\section{REFERENCES}

1. Amarillo National Resource Center for Plutonium. 1996. Fiscal Year 1997 Continuation Application (abbr. ver.) for the Amarillo National Resource Center for Plutonium.

2. Bejan, Adrian. 1987. "Buckling flows: a new frontier in fluid mechanics." Annual Review of Numerical Fluid Mechanics and Heat Transfer, vol. 1, pp. 262-304.

3. Buckmaster, J. 1973. "The buckling of thin viscous jets." Journal of Fluid Mechanics, vol. 61, part 3, pp. 449-463.

4. Chacha, M., Occelli, R., and Tadrist, L. 1994. "Heat transfer in high-temperature liquid jets." International Journal of Heat and Mass Transfer, vol. 37, no. 18, pp. 2871-2883.

5. Chu, T. Y., and Hickox, C. E. 1990. "Thermal convection with large viscosity variation in an enclosure with localized heating." Journal of Heat Transfer, vol. 112, pp. 388-395.

6. Cruickshank, J. O., and Munson, B. R. 1981. "Viscous fluid buckling of plane and axisymmetric jets." Journal of Fluid Mechanics, vol. 113, pp. 221-239.

7. Dow Corning Corporation. 1980. Information about silicone fluids. Midland, MI.

8. Flow Science, Inc. FLOW-3D Quick Reference Guide.

9. Flow Science, Inc. 1996. FLOW-3D Theory Manual.

10. Fluid Dynamics International, Inc. 1993. FIDAP 7.0 FIPREP Users Manual, rev. 7.0, first ed.
11. Fluid Dynamics International, Inc. 1993. FIDAP 7.0 Theory Manual, rev. 7.0, first ed.

12. Fluid Dynamics International, Inc. 1995. FIDAP 7.5 Update Manual, rev. 7.5, first ed.

13. Fluid Dynamics International, Inc. 1996. FIDAP 7.6 Update Manual, rev. 7.6, first ed.

14. Gomon, Michael. 1997. "Experimental study of the behavior of highly viscous impinging jets." Thesis, The University of Texas at Austin.

15. Hirt, C. W., and Sicilian, J. M. 1985. “A porosity technique for the definition of obstacles in rectangular cell meshes." Proceedings of the Fourth International Conference on Ship Hydrodynamics, National Academy of Science, Washington, D.C. Referenced in FLOW$3 D$ Quick Reference Guide, Flow Science, Inc.

16. Panton, Ronald L. 1996. Incompressible Flow, second ed. New York: John Wiley \& Sons, Inc.

17. Reddy, J. N., and Gartling, D. K. 1994. The finite element method in heat transfer and fluid dynamics. Boca Raton, FL: CRC Press, Inc.

18. Soper, P. D., and Bickford, D. F. 1982. Physical properties of frit 165/waste glasses. DPST-82-899, Technical Division - Savannah River Laboratory, Aiken, SC. Referenced in Jerrell, J. W., and Hardy, B. J., 1995, Estimates of glass temperatures and viscosities during filling of can-in-can assembly, WSRC-TR-950416, Westinghouse Savannah River Company, Savannah River Technology Center. 
19. Suleiman, S. M., and Munson, B. R. 1981. "Buckling of a thin sheet of a viscous fluid." Physics of Fluids, vol. 24, pp. 1-5.

20. Taylor, G. I. 1969. "Instability of jets, threads, and sheets of viscous fluid." Proceedings of the 12th International Congress of Applied Mechanics (Stanford, 1968), pp. 382-388.
21. Tchavdarov, B., Yarin, A. L., and Radev, S. 1993. "Buckling of thin liquid jets." Journal of Fluid Mechanics, vol. 253, pp. 593-615.

22. Viskanta, R. 1994. "Review of threedimensional mathematical modeling of glass melting." Journal of NonCrystalline Solids, vol. 177, pp. 347-362. 


\section{APPENDIX A Brief Guide to FLOW-3D}

The FLOW-3D software package consists of three main modules: PREP3D is the preprocessor (sets up the geometry, grid, property data, and boundary and initial conditions); HYDR3D is the solver (calculates flow solution at each time step); and FLSCON is the postprocessor (creates requested plots of the results). Problems are run using one of several scripts. For regular runs (starting from $t=0$ ), these scripts are runpre (runs the preprocessor PREP3D only), runhyd (runs the solver HYDR3D only), runpost (runs the postprocessor FLSCON only), and runall (runs the three modules consecutively). There are corresponding scripts for restart runs (starting from $t>0$ ): respre, reshyd, respost, and resall.

Each of the program modules requires certain input. PREP3D takes its input from the user-created ASCII file prepin. inp (or prepinr. inp for restart runs), and creates the files necessary for HYDR3D to run. HYDR3D takes these input files, solves the problem, and outputs the results to FLSCON, which creates a file containing all the plots requested by the user. These plots may be viewed using one of the graphics display programs provided (display or pltfsi).

To solve a problem using FLOW-3D, move into the directory containing the prepin.inp file, and type runall. The preprocessing, solution, and postprocessing phases will execute, and then the results may be viewed by typing pltfsi and following the menu instructions.

All of the problem specifications are included in the prepin. inp file: geometry, grid, physical and computational models, fluid and solid properties, boundary and initial conditions, and plot requests. This input file consists of several "blocks" of commands. These blocks break the input file into logical groups of commands, so for example, all the boundary condition commands are together in one block. Every FLOW-3D command belongs in a specific block, and cannot be placed in any of the other blocks. The commands within a block may be placed in any order, and not all the available commands in a block must be included - if a command is not included, its default value will be assumed. However, the blocks themselves must be placed in a specific order, and almost all of the blocks must be included in the input file, even if they contain no commands. Each block begins with the $\$$ character and the name of the block, and ends with the \$end command. Every command must be followed by a comma, except for the commands to begin and end a block. Multiple commands may be placed on one line, as long as each command is followed by a comma. FLOW3D ignores any characters in the first column of the input file, so no text should be placed in this first column. Any text between blocks is ignored by FLOW-3D.

Following is a sample FLOW-3D input file prepin. inp, along with an explanation of the file's contents and the various FLOW-3D options which are used. This input file will set up the "base case" of the basic model from section 4.3.2, which was used to evaluate the robustness of the solution. (The line numbers in the left margin are for reference only, and are not part of the input file.) 
(01) Highly viscous impinging jet -- base case

(02)

(03)

(04)

(05)

(06)

(07)

(08)

(09)

(10)

(11)

(12)

(13)

(14)

(15)

(16)

(17)

(18)

(19)

(20)

(21)

(22)

(23)

(24)

(25)

(26)

(27)

(28)

(29)

(30)

(31)

(32)

(33)

(34)

(35)

(36)

(37)

(38)

(39)

(40)

(41)

(42)

(43)

(44)

(45)

(46)

(47)

(48)

(49)

(50)

(51)

(52)

(53)

(54)

\$xput

remark='units are cgs',

twfin $=2 . d 0$,

pltat $=0.01 \mathrm{~d} 0$,

nmat $=1$,

$i t b=1$,

iorder $=3$,

impvis $=1$,

iadix $=1$,

epsadj=0.1d0, $g z=-980.7 d 0$,

send

\$limits

itvsm $=500$,

send

\$props

remark='for 60,000 cSt silicone oil',

rhof $=0.976 \mathrm{do}$,

mui $=585.6 \mathrm{do}$,

sigma $=21.5 \mathrm{do}$,

cangle $=90 . d 0$,

send

$\$$ bcdata

$\mathrm{w} l=2$,

$w r=2$,

$w b=2$,

$\mathrm{wt}=6$,

$\operatorname{wbc}(6)=-34.89 \mathrm{do}$,

send

\$mesh

icy $1=0$,

$\mathrm{px}(1)=-7.62 \mathrm{do}$,

$\mathrm{px}(2)=-0.59 \mathrm{d0}$ ，

$\mathrm{px}(3)=0.59 \mathrm{do}$,

$\operatorname{px}(4)=7.62 \mathrm{do}$,

$\mathrm{pz}(1)=0 . \mathrm{d} 0$,

$\mathrm{pz}(2)=13.692 \mathrm{do}$,

$p z(3)=34.23 \mathrm{do}$,

nxcelt $=41$,

nzcelt $=80$,

nxcell $(2)=9$,

nzcell $(1)=48$,

send

Sobs

avrck $=-2.1$,

send 


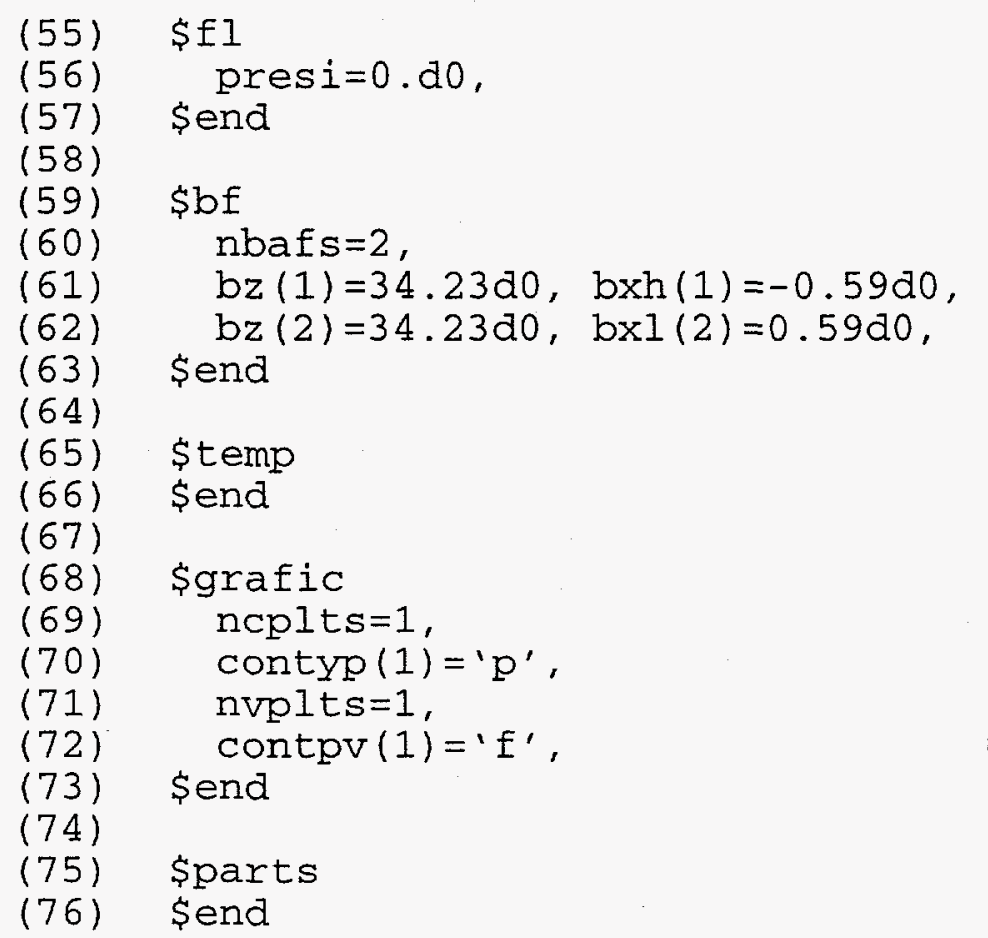

(01) Any text on the first line of the input file is considered to be the title, and will be printed at the bottom of all plots generated. Any other text which is placed before the beginning of the first block will be ignored by FLOW-3D.

(03) The \$xput command marks the beginning of the "xput". block, in which the physical and computational parameters of the problem are specified.

(04) The remark command may be used anywhere in the file. The argument of this command, placed within quotation marks, will be ignored by FLOW-3D.

(05) This specifies the ending time of the calculation. When the problem reaches this time, the solution will end and plots will be generated.

(06) This specifies the time interval between plots of the solution.

(07) This specifies the number of materials present in the problem. Since this "base case" is a single fluid model, nmat is set equal to one.

(08) This indicates that there is a sharp, precise free surface between the fluid and the constant-pressure void. If $i t b$ were set equal to zero, FLOW-3D would assume the fluid and void could mix freely.

(09) This indicates that a monotonicity-preserving second order approximation should be used for the advective terms in the momentum equation. This option was chosen because it is much more accurate than the default first-order approximation, and uses only slightly more CPU time. For more information on this option, see pp. 35 - 40 of the FLOW-3D Theory Manual.

(10) This enables the implicit solution of the viscous stress term, as discussed in sections 4.3.2 and 4.3.4. For more information, see the FLOW-3D Theory Manual, pp. 61 - 63.

(11) This indicates that the alternating direction implicit method should be used to calculate the pressure in the $x$ direction. This option was enabled because of the symmetry in this direction, and because of the large difference between the horizontal width of the elements in the center of the domain and the elements near the walls. See the FLOW-3D Theory Manual, pp. 49 - 53, for more information. (Because the command 
iadi $z=1$ was not used, the pressure in the $z$ direction will be calculated using the default successive over-relaxation method.)

(12) As discussed in Section 4.3.2, this option causes the convergence criterion for the pressure iterations to be "tighter" than the default value by one order of magnitude.

(13) This command specifies the gravitational acceleration in the $z$ direction. Since the cgs system of units was chosen, the acceleration must be specified in units of $\mathrm{cm}^{2} / \mathrm{s}$.

(14) The send command indicates that all of the commands in the "xput" block have been entered.

(16) The "limits" block contains information on computational limits.

(17) This command sets the maximum number of iterations allowed when calculating the viscous stress, as discussed in Section 4.3.2.

(20) The "props" block contains specifications of the fluid properties.

(22) The density of $60,000 \mathrm{cSt}$ silicone oil, in $\mathrm{g} / \mathrm{cm}^{3}$, is specified.

(23) The dynamic viscosity of the silicone oil, in $\mathrm{g} / \mathrm{cm} \cdot \mathrm{s}$, is specified.

(24) The surface tension of the silicone oil - air interface is specified, in units of $\mathrm{g} / \mathrm{s}^{2}$.

(25) The contact angle between the silicone oil and the wall of the receiving container, in degrees, is specified.

(28) The "bcdata" block contains specifications of the boundary conditions.

(29) The variable wl sets the condition type at the left boundary of the domain. Type 2 is a rigid (no-slip) wall. Setting $w r=2$ and $w b=2$ indicates that the right and bottom boundaries, respectively, are also no-slip walls. The "symmetry" boundary condition is applied to the front and back walls by default.

(32) This command indicates that the top boundary of the domain has a specified velocity.
(33) This specifies the vertical velocity of the fluid at the top boundary. The units are $\mathrm{cm} / \mathrm{s}$. The name of the command (wbc) indicates that the command is specifying the $z$ component of velocity (ubc sets the $x$ velocity, and vbc sets the $y$ velocity), and the argument (6) indicates that this velocity is to be applied to the top boundary $(1=$ left boundary, $2=$ right, $3=$ front, $4=$ back, and $5=$ bottom).

(36) The "mesh" block contains information about the physical geometry and the computational grid.

(37) Setting icy $1=0$ indicates that the problem is specified in terms of Cartesian coordinates.

(38) The array px defines the $x$ coordinates of points along a horizontal line. The user may specify as many px points as are necessary to define the domain. They must proceed in order from left to right. In this case, $\mathrm{px}(1)$ is used to mark the left edge of the domain, $\mathrm{px}(2)$ marks the left edge of the jet inlet, $\mathrm{px}$ (3) marks the right edge of the jet inlet, and $\mathrm{px}(4)$ marks the right edge of the domain.

(42) The pz array defines the $z$ coordinates of points along a vertical line, in order from bottom to top. For this input file, $\mathrm{pz}$ (1) marks the bottom edge of the domain, $p z$ (2) marks a point $40 \%$ of the way up the domain, and $p z$ (3) marks the top edge of the domain.

(45) This variable specifies the total number of computational cells in the $x$ direction.

(46) The total number of computational cells in the $z$ direction are specified by this variable.

(47) This variable specifies the number of cells to be placed in "section 2" of the $x$ grid direction, i.e. between $\mathrm{px}(2)$ and $\mathrm{px}(3)$ (across the jet width).

(48) This specifies the number of cells in "section 1 " of the $z$ grid direction, i.e. between $p z$ (1) and $p z$ (2) (the bottom $40 \%$ of the domain). 
(51) The "obs" block contains information on $2 \mathrm{D}$ or $3 \mathrm{D}$ solid obstacles.

(52) The variable avrck adjusts the maximum area/volume ratio for every cell so that the ratio does not exceed this value. This is done to prevent computational cells that happen to lie along a computational grid line from severely limiting the size of the time step. For more information on the area and volume fractions and the FAVOR method, see pp. 31 - 34 of the FLOW-3D Theory Manual, or Hirt and Sicilian (1985).

(55) The "fl" block contains initial conditions for the fluid.

(56) This command sets the initial pressure in the entire domain to zero.

(59) The "bf' block contains specifications for 1D or 2D baffles.

(60) This command sets the number of baffles in the problem.

(61) These two commands specify the $z$ coordinate of the first baffle, and the maximum $x$ coordinate of this baffle. For this particular input, baffle \#1 will be a horizontal wall blocking the top boundary of the domain from $x=-\infty$ to $x=-0.59$ (the left edge of the jet inlet).

(62) These two commands specify the $z$ coordinate of the second baffle, and the minimum $x$ coordinate of this baffle. For this particular input, baffle \#2 will be a horizontal wall blocking the top boundary of the domain from $x=0.59$ (the right edge of the jet inlet) to $x=\infty$. Thus the entire top boundary is blocked off, except for an area in the center of the boundary where the jet is allowed to flow into the domain with the velocity specified in line 33.

(65) The "temp" block specifies initial conditions for temperature and heat transfer. Since this "base case" has no heat transfer, this block contains no commands, but it must still be included in the input file.

(68) The "grafic" block contains requests for plots to be generated. When the FLSCON postprocessor is executed, it will generate all the plots requested in this block and write them to a file, so they may be viewed using the pltfsi or display programs.

(69) This command specifies the number of contour plots requested.

(70) This command indicates that the first (and in this case, only) contour plot should show contours of the pressure (indicated by the ' $p$ '). The plot will have solid color contours of pressure as a background, with ten pressure contour lines superimposed.

(71) This specifies that one vector plot is requested.

(72) This command indicates that the vector plot should show contours of the fill fraction (indicated by the ' $f$ '). The plot will have solid color contours of the fill fraction as a background, with the free surface ( $F=0.5$ contour line) and velocity vectors superimposed.

(74) The "parts" block specifies particle tracking options. No particles were tracked in these simulations.

For more information on the FLOW$3 \mathrm{D}$ program structure, or any of these commands, see Chapters 1 and 2 of the FLOW-3D Quick Reference Guide. 